УДК 517.553

\author{
A. I. BAndura, O. B. Skaskiv
}

\title{
ANALYTIC FUNCTIONS IN THE UNIT BALL OF BOUNDED L-INDEX: ASYMPTOTIC AND LOCAL PROPERTIES
}

\begin{abstract}
A. I. Bandura, O. B. Skaskiv. Analytic functions in the unit ball of bounded $\mathbf{L}$-index: asymptotic and local properties, Mat. Stud. 48 (2017), 37-73.

We have generalized some criteria of boundedness of $\mathbf{L}$-index in joint variables for analytic functions in the unit ball, where $\mathbf{L}: \mathbb{B}^{n} \rightarrow \mathbb{R}_{+}^{n}$ is a continuous vector-function, $\mathbb{B}^{n}$ is the unit ball in $\mathbb{C}^{n}$. One of propositions gives an estimate of the coefficients of power series expansions by a dominating homogeneous polynomial for analytic functions in the unit ball. Also we provide growth estimates of these functions. They describe the behavior of maximum modulus of analytic function on a skeleton in a polydisc by behavior of the function $\mathbf{L}$.

Most of our results are based on polydisc exhaustion of the unit ball. Nevertheless, we have generalized criteria of boundedness of $\mathbf{L}$-index in joint variables which describe local behavior of partial derivatives on sphere in $\mathbb{C}^{n}$. The proposition uses a ball exhaustion.

An analog of Hayman's theorem is applied to investigation of boundedness of L-index in joint variables for analytic solutions in the unit ball of some linear higher-order systems of PDE's. There were found sufficient conditions providing the boundedness. Growth estimates of analytic solutions in the unit ball are also obtained.
\end{abstract}

1. Introduction. The most important classes of analytic functions of several variables are analytic functions in the polydisc and analytic functions in the unit ball. In our investigations, we develop theory of functions of bounded index for these classes ([1,2,12-14]). A concept of bounded index is very flexible to investigate properties of analytic solutions of ordinary and partial differential equations and its systems ([7]).

The paper is a continuation of our investigations from [1,2]. There was introduced a concept of analytic function of bounded $\mathbf{L}$-index in joint variables in a ball in $\mathbb{C}^{n}$. We obtained some criteria of L-index boundedness in joint variables. They describe local behavior of partial derivatives and maximum modulus of analytic functions in the unit ball. They are generalizations of corresponding theorems which are known for entire functions of several variables $([9,10,29])$.

In [2], we announced a possibility of application of Hayman's theorem to linear higherorder system of PDE's whose coefficients are analytic functions in the unit ball. There was presented an application scheme to a special system. Now, we consider a more general system of PDE's. Besides, some asymptotic estimates for the class are deduced. They describe growth of the logarithm of the maximum modulus of an analytic function on the skeleton of a polydisc via the behavior of some continuous vector function $\mathbf{L}: \mathbb{C}^{n} \rightarrow \mathbb{R}_{+}^{n}$.

2010 Mathematics Subject Classification: 32A05, 32A10, 32A22, 35G35, 32A40, 32W50.

Keywords: analytic function in a ball; bounded index in joint variables; maximum modulus; partial derivative; Cauchy's integral formula; geometric exhaustion; growth estimates; linear higher-order systems of PDE. doi: $10.15330 / \mathrm{ms} .48 .1 .37-73$

(C) A. I. Bandura, O. B. Skaskiv, 2017 
The main method of our investigations uses a polydisc exhaustion of the unit ball. It is very convenient for the concept of bounded $\mathbf{L}$-index in joint variables and functions of several variables. Nevertheless, a ball exhaustion seems to be a more natural approach for analytic function in the unit ball than a polydisc exhaustion. Thus, we also have established some results about local behavior of partial derivatives for functions of this class which are based on Cauchy's integral formula for a ball.

There is another approach to introduce a concept of bounded index in $\mathbb{C}^{n}$ and $\mathbb{B}^{n}$. It uses a slice function. These functions are called functions of bounded $L$-index in direction. They are considered in $[3,5,6,8,16]$.

In view of results from $[18,19]$ it is not difficult to prove that for every analytic function $F$ in the unit ball with bounded multiplicities there exists a continuous function $\mathbf{L}: \mathbb{C}^{n} \rightarrow \mathbb{R}_{+}$ such that $F$ is of bounded $\mathbf{L}$-index in joint variables. Thus, the class of analytic function in the unit ball of bounded $\mathbf{L}$-index in joint variables is very wide.

2. Main definitions and notations. We need some standard notations. Denote

$$
\begin{gathered}
\mathbb{R}_{+}=[0,+\infty), \mathbf{0}=(0, \ldots, 0) \in \mathbb{R}_{+}^{n}, \mathbf{1}=(1, \ldots, 1) \in \mathbb{R}_{+}^{n}, \\
\mathbf{1}_{j}=(0, \ldots, 0, \underbrace{1}_{j-\text { th place }}, 0, \ldots, 0) \in \mathbb{R}_{+}^{n}, R=\left(r_{1}, \ldots, r_{n}\right) \in \mathbb{R}_{+}^{n}, \\
z=\left(z_{1}, \ldots, z_{n}\right) \in \mathbb{C}^{n},|z|=\sqrt{\sum_{j=1}^{n}\left|z_{j}\right|^{2} .}
\end{gathered}
$$

For $A=\left(a_{1}, \ldots, a_{n}\right) \in \mathbb{R}^{n}, B=\left(b_{1}, \ldots, b_{n}\right) \in \mathbb{R}^{n}$ we will use formal notations without violation of the existence of these expressions $A B=\left(a_{1} b_{1}, \cdots, a_{n} b_{n}\right), A / B=\left(a_{1} / b_{1}, \ldots, a_{n} / b_{n}\right)$, $A^{B}=a_{1}^{b_{1}} a_{2}^{b_{2}} \cdot \ldots \cdot a_{n}^{b_{n}},\|A\|=a_{1}+\cdots+a_{n}$, and the notation $A<B$ means that $a_{j}<b_{j}$, $j \in\{1, \ldots, n\}$; the relation $A \leq B$ is defined similarly. For $K=\left(k_{1}, \ldots, k_{n}\right) \in \mathbb{Z}_{+}^{n}$ denote $K !=k_{1} ! \cdot \ldots \cdot k_{n} !$. Addition, scalar multiplication, and conjugation are defined on $\mathbb{C}^{n}$ componentwise. For $z \in \mathbb{C}^{n}$ and $w \in \mathbb{C}^{n}$ we define

$$
\langle z, w\rangle=z_{1} \bar{w}_{1}+\cdots+z_{n} \bar{w}_{n},
$$

where $w_{k}$ is the complex conjugate of $w_{k}$. The polydisc $\left\{z \in \mathbb{C}^{n}:\left|z_{j}-z_{j}^{0}\right|<r_{j}, j=1, \ldots, n\right\}$ is denoted by $\mathbb{D}^{n}\left(z^{0}, R\right)$, its skeleton $\left\{z \in \mathbb{C}^{n}:\left|z_{j}-z_{j}^{0}\right|=r_{j}, j=1, \ldots, n\right\}$ is denoted by $\mathbb{T}^{n}\left(z^{0}, R\right)$, and the closed polydisc $\left\{z \in \mathbb{C}^{n}:\left|z_{j}-z_{j}^{0}\right| \leq r_{j}, j=1, \ldots, n\right\}$ is denoted by $\mathbb{D}^{n}\left[z^{0}, R\right], \mathbb{D}^{n}=\mathbb{D}^{n}(\mathbf{0}, \mathbf{1}), \mathbb{D}=\{z \in \mathbb{C}:|z|<1\}$. The open ball $\left\{z \in \mathbb{C}^{n}:\left|z-z^{0}\right|<r\right\}$ is denoted by $\mathbb{B}^{n}\left(z^{0}, r\right)$, its boundary is a sphere $\mathbb{S}^{n}\left(z^{0}, r\right)=\left\{z \in \mathbb{C}^{n}:\left|z-z^{0}\right|=r\right\}$, the closed ball $\left\{z \in \mathbb{C}^{n}:\left|z-z^{0}\right| \leq r\right\}$ is denoted by $\mathbb{B}^{n}\left[z^{0}, r\right], \mathbb{B}^{n}=\mathbb{B}^{n}(\mathbf{0}, 1), \mathbb{D}=\mathbb{B}^{1}=\{z \in \mathbb{C}:|z|<1\}$.

For $K=\left(k_{1}, \ldots, k_{n}\right) \in \mathbb{Z}_{+}^{n}$ and the partial derivatives of an analytic function $F(z)=$ $F\left(z_{1}, \ldots, z_{n}\right)$ in $\mathbb{B}^{n}$ we use the notation

$$
F^{(K)}(z)=\frac{\partial^{\|K\|} F}{\partial z^{K}}=\frac{\partial^{k_{1}+\cdots+k_{n}} f}{\partial z_{1}^{k_{1}} \ldots \partial z_{n}^{k_{n}}} .
$$

Let $\mathbf{L}(z)=\left(l_{1}(z), \ldots, l_{n}(z)\right)$, where $l_{j}(z): \mathbb{B}^{n} \rightarrow \mathbb{R}_{+}$is a continuous function such that

$$
\left(\forall z \in \mathbb{B}^{n}\right): l_{j}(z)>\beta /(1-|z|), j \in\{1, \ldots, n\},
$$

where $\beta>\sqrt{n}$ is a some constant. 
S. N. Strochyk, M. M. Sheremeta, V. O. Kushnir ([25,35,36]) imposed a similar condition for a function $l: \mathbb{D} \rightarrow \mathbb{R}_{+}$and $l: G \rightarrow \mathbb{R}_{+}$, where $G$ is an arbitrary domain in $\mathbb{C}$.

An analytic function $F: \mathbb{B}^{n} \rightarrow \mathbb{C}$ is said $([1,2])$ to be of bounded $\mathbf{L}$-index (in joint variables), if there exists $n_{0} \in \mathbb{Z}_{+}$such that for all $z \in \mathbb{B}^{n}$ and for all $J \in \mathbb{Z}_{+}^{n}$

$$
\frac{\left|F^{(J)}(z)\right|}{J ! \mathbf{L}^{J}(z)} \leq \max \left\{\frac{\left|F^{(K)}(z)\right|}{K ! \mathbf{L}^{K}(z)}: K \in \mathbb{Z}_{+}^{n},\|K\| \leq n_{0}\right\}
$$

The least such integer $n_{0}$ is called the $\mathbf{L}$-index in joint variables of the function $F$ and is denoted by $N\left(F, \mathbf{L}, \mathbb{B}^{n}\right)$. There are many papers about entire functions of several variables of bounded index $([21,22,24,27-29])$ and of bounded $\mathbf{L}$-index in joint variables $([5,9-11,17])$.

By $Q\left(\mathbb{B}^{n}\right)$ we denote the class of functions $\mathbf{L}$, satisfying (1) and the following condition

$$
\left(\forall R \in \mathbb{R}_{+}^{n},|R| \leq \beta, j \in\{1, \ldots, n\}\right): 0<\lambda_{1, j}(R) \leq \lambda_{2, j}(R)<\infty,
$$

where

$$
\begin{gathered}
\lambda_{1, j}(R)=\inf _{z^{0} \in \mathbb{B}^{n}} \inf \left\{\frac{l_{j}(z)}{l_{j}\left(z^{0}\right)}: z \in \mathbb{D}^{n}\left[z^{0}, R / \mathbf{L}\left(z^{0}\right)\right]\right\}, \\
\lambda_{2, j}(R)=\sup _{z^{0} \in \mathbb{B}^{n}} \sup \left\{\frac{l_{j}(z)}{l_{j}\left(z^{0}\right)}: z \in \mathbb{D}^{n}\left[z^{0}, R / \mathbf{L}\left(z^{0}\right)\right]\right\} . \\
\Lambda_{1}(R)=\left(\lambda_{1,1}(R), \ldots, \lambda_{1, n}(R)\right), \Lambda_{2}(R)=\left(\lambda_{2,1}(R), \ldots, \lambda_{2, n}(R)\right) .
\end{gathered}
$$

It is not difficult to verify that the class $Q\left(\mathbb{B}^{n}\right)$ can be defined as following

$$
\text { for every } j \in\{1, \ldots, n\} \sup _{z, w \in \mathbb{B}^{n}}\left\{\frac{l_{j}(z)}{l_{j}(w)}:\left|z_{k}-w_{k}\right| \leq \frac{r_{k}}{\min \left\{l_{k}(z), l_{k}(w)\right\}}, k \in\{1, \ldots, n\}\right\}<\infty \text {, }
$$

i. e. conditions (3) and (7) are equivalent (see a definition of a similar class for $\mathbb{C}^{n}$ in $[6]$ ).

We also need the following assertions. They are generalizations of corresponding propositions for entire functions of bounded $L$-index in direction $[3,16]$ and of bounded $\mathbf{L}$-index in joint variables $([10,17])$ and of bounded index $([23])$.

Theorem 1 ([2]). Let $\mathbf{L} \in Q\left(\mathbb{B}^{n}\right)$. An analytic function $F$ in $\mathbb{B}^{n}$ has bounded $\mathbf{L}$-index in joint variables if and only if there exist $p \in \mathbb{Z}_{+}$and $c \in \mathbb{R}_{+}$such that for each $z \in \mathbb{B}^{n}$

$$
\max \left\{\frac{\left|F^{(J)}(z)\right|}{\mathbf{L}^{J}(z)}:\|J\|=p+1\right\} \leq c \cdot \max \left\{\frac{\left|F^{(K)}(z)\right|}{\mathbf{L}^{K}(z)}:\|K\| \leq p\right\} .
$$

Theorem $\mathbf{2}([2])$. Let $\mathbf{L} \in Q^{n}, F: \mathbb{B}^{n} \rightarrow \mathbb{C}$ be an analytic function. If there exist $R^{\prime}$, $R^{\prime \prime} \in \mathbb{R}_{+}^{n}, \mathbf{0}<R^{\prime}<R^{\prime \prime},\left|R^{\prime \prime}\right|<\beta$ and $p_{1}=p_{1}\left(R^{\prime}, R^{\prime \prime}\right) \geq 1$ such that for every $z^{0} \in \mathbb{C}^{n}$ inequality

$$
\max \left\{|F(z)|: z \in \mathbb{T}^{n}\left(z^{0}, \frac{R^{\prime \prime}}{\mathbf{L}\left(z^{0}\right)}\right)\right\} \leq p_{1} \max \left\{|F(z)|: z \in \mathbb{T}^{n}\left(z^{0}, \frac{R^{\prime}}{\mathbf{L}\left(z^{0}\right)}\right)\right\}
$$

holds then the function $F$ is of bounded $\mathbf{L}$-index in joint variables. 
3. Properties of power series expansion of analytic functions in the unit ball. Let $z^{0} \in \mathbb{B}^{n}$. We develop an analytic function $F: \mathbb{B}^{n} \rightarrow \mathbb{C}$ in the power series written in a diagonal form

$$
F(z)=\sum_{k=0}^{\infty} p_{k}\left(z-z^{0}\right)=\sum_{k=0}^{\infty} \sum_{\|J\|=k} b_{J}\left(z-z^{0}\right)^{J}
$$

where $p_{k}$ are homogeneous polynomials of $k$-th degree, $b_{J}=\frac{F^{(J)}\left(z^{0}\right)}{J !}$. A polynomial $p_{k_{0}}, k_{0} \in$ $\mathbb{Z}_{+}$, is called a dominating polynomial in the power series expansion $(10)$ on $\mathbb{T}^{n}\left(z^{0}, R\right)$ if for every $z \in \mathbb{T}^{n}\left(z^{0}, R\right)$ the next inequality holds:

$$
\left|\sum_{k \neq k^{0}} p_{k}\left(z-z^{0}\right)\right| \leq \frac{1}{2} \max \left\{\left|b_{J}\right| R^{J}:\|J\|=k^{0}\right\} .
$$

Recently, Theorems 3 and 4 were obtained for entire functions ([11]) and for analytic function in a bidisc ([14]). Now we deduce these propositions for analytic functions in the unit ball.

Theorem 3. Let $\mathbf{L} \in Q\left(\mathbb{B}^{n}\right)$. If an analytic function $F$ in $\mathbb{B}^{n}$ has bounded $\mathbf{L}$-index in joint variables then there exists $p \in \mathbb{Z}_{+}$that for all $d \in\left(0 ; \frac{\beta}{\sqrt{n}}\right]$ there exists $\eta(d) \in(0 ; d)$ such that for each $z^{0} \in \mathbb{B}^{n}$ and some $r=r\left(d, z^{0}\right) \in(\eta(d), d), k^{0}=k^{0}\left(d, z^{0}\right) \leq p$ the polynomial $p_{k^{0}}$ is a dominating polynomial in the series $(10)$ on $\mathbb{T}^{n}\left(z^{0}, \frac{r \mathbf{1}}{\mathbf{L}\left(z^{0}\right)}\right)$.

Proof. Let $F$ be an analytic function of bounded $\mathbf{L}$-index in joint variables with $N=$ $N\left(F, \mathbf{L}, \mathbb{B}^{n}\right)<+\infty$ and $n_{0}$ be the $\mathbf{L}$-index in joint variables at a point $z^{0} \in \mathbb{D}^{2}$, i.e. $n_{0}$ is the least number, for which inequality (2) holds at the point $z^{0}$. Then for each $z^{0} \in \mathbb{B}^{n}$ $n_{0} \leq N$.

We put

$$
\begin{gathered}
a_{J}^{*}=\frac{\left|b_{J}\right|}{\mathbf{L}^{J}\left(z^{0}\right)}=\frac{\left|F^{(J)}\left(z^{0}\right)\right|}{J ! \mathbf{L}^{J}\left(z^{0}\right)}, \\
a_{k}=\max \left\{a_{J}^{*}:\|J\|=k\right\}, c=2\left\{(N+n+1) !(n+1) !+(N+1) C_{n+N-1}^{N}\right\} .
\end{gathered}
$$

Let $d \in\left(0 ; \frac{\beta}{\sqrt{n}}\right]$ be an arbitrary number. We also denote $r_{m}=\frac{d}{(d+1) c^{m}}, \mu_{m}=\max \left\{a_{k} r_{m}^{k}: k \in\right.$ $\left.\mathbb{Z}_{+}\right\}, s_{m}=\min \left\{k: a_{k} r_{m}^{k}=\mu_{m}\right\}$ for $m \in \mathbb{Z}_{+}$.

Since $z^{0} \in \mathbb{B}^{n}$ is a fixed point the inequality $a_{K}^{*} \leq \max \left\{a_{J}^{*}:\|J\| \leq n_{0}\right\}$ is valid for all $K \in \mathbb{Z}_{+}^{n}$. Then $a_{k} \leq a_{n_{0}}$ for all $k \in \mathbb{Z}_{+}$. Hence, for all $k>n_{0}$, in view of $r_{0}<1$, we have $a_{k} r_{0}^{k}<a_{n_{0}} r_{0}^{n_{0}}$. This implies $s_{0} \leq n_{0}$. Since $c r_{m}=r_{m-1}$, we obtain that for each $k>s_{m-1}$ $\left(r_{m-1}<1\right)$

$$
a_{s_{m-1}} r_{m}^{s_{m-1}}=a_{s_{m-1}} r_{m-1}^{s_{m-1}} c^{-s_{m-1}} \geq a_{k} r_{m-1}^{k} c^{-s_{m-1}}=a_{k} r_{m}^{k} c^{k-s_{m-1}} \geq c a_{k} r_{m}^{k} .
$$

It yields that $s_{m} \leq s_{m-1}$ for all $m \in \mathbb{N}$. Thus, we can rewrite

$$
\mu_{0}=\max \left\{a_{k} r_{0}^{k}: k \leq n_{0}\right\}, \mu_{m}=\max \left\{a_{k} r_{m}^{k}: k \leq s_{m-1}\right\}, m \in \mathbb{N} .
$$

Let us introduce additional notations for $m \in \mathbb{N}$

$$
\mu_{0}^{*}=\max \left\{a_{k} r_{0}^{k}: s_{0} \neq k \leq n_{0}\right\}, s_{0}^{*}=\min \left\{k: k \neq s_{0}, a_{k} r_{0}^{k}=\mu_{0}^{*}\right\},
$$




$$
\mu_{m}^{*}=\max \left\{a_{k} r_{m}^{k}: s_{m} \neq k \leq s_{m-1}\right\}, s_{m}^{*}=\min \left\{k: k \neq s_{m}, a_{k} r_{m}^{k}=\mu_{m}^{*}\right\} .
$$

We will show that there exists $m_{0} \in \mathbb{Z}_{+}$such that

$$
\frac{\mu_{m_{0}}^{*}}{\mu_{m_{0}}} \leq \frac{1}{c}
$$

Suppose that for all $m \in \mathbb{Z}_{+}$the next inequality holds

$$
\frac{\mu_{m}^{*}}{\mu_{m}}>\frac{1}{c}
$$

If $s_{m}^{*}<s_{m}\left(s_{m}^{*} \neq s_{m}\right.$ in view of definition) then we have

$$
a_{s_{m}^{*}} r_{m+1}^{s_{m}^{*}}=\frac{a_{s_{m}^{*}} r_{m}^{s_{m}^{*}}}{c^{s_{m}^{*}}}=\frac{\mu_{m}^{*}}{c^{s_{m}^{*}}}>\frac{\mu_{m}}{c^{s_{m}^{*}+1}}=\frac{a_{s_{m}} r_{m}^{s_{m}}}{c^{s_{m}^{*}+1}}=\frac{a_{s_{m}} r_{m+1}^{s_{m}}}{c^{s_{m}^{*}+1-s_{m}}} \geq a_{s_{m}} r_{m+1}^{s_{m}}
$$

Besides, for every $k>s_{m}^{*}, k \neq s_{m}$, (i. e., $k-1 \geq s_{m}^{*}$ ) it can be deduced similarly that

$$
a_{s_{m}^{*}} r_{m+1}^{s_{m}^{*}}=\frac{a_{s_{m}^{*}} r_{m}^{s_{m}^{*}}}{c^{s_{m}^{*}}} \geq \frac{a_{k} r_{m}^{k}}{c^{s_{m}^{*}}} \geq \frac{a_{k} r_{m}^{k}}{c^{k-1}}=c a_{k} r_{m+1}^{k}
$$

Hence, $a_{s_{m}^{*}} r_{m+1}^{s_{m}^{*}}>a_{k} r_{m+1}^{k}$ for all $k>s_{m}^{*}$. Then

$$
s_{m+1} \leq s_{m}^{*} \leq s_{m}-1
$$

On the contrary, if $s_{m}<s_{m}^{*} \leq s_{m-1}$, then the equality $s_{m+1}=s_{m}$ may holds. Indeed, by definition $s_{m+1} \leq s_{m}$. It means that the specified equality is possible. But if $s_{m+1}<s_{m}$ then $s_{m+1} \leq s_{m}-1$ (they are natural numbers!). Hence, we obtain (14).

Thus, the inequalities $s_{m+1}^{*} \leq s_{m}$ and $s_{m}^{*} \neq s_{m+1}$ imply that $s_{m+1}^{*}<s_{m+1}$. As above instead of (14) we have

$$
s_{m+2} \leq s_{m+1}^{*} \leq s_{m+1}-1=s_{m}-1 .
$$

Therefore, if for all $m \in \mathbb{Z}_{+}$(13) holds, then for every $m \in \mathbb{Z}_{+}$either $s_{m+2} \leq s_{m+1} \leq$ $s_{m}-1$ or $s_{m+2} \leq s_{m}-1$ holds, that is $s_{m+2} \leq s_{m}-1$, because $s_{m+2} \leq s_{m+1}$. It follows that

$$
s_{m} \leq s_{m-2}-1 \leq \ldots \leq s_{m-2[m / 2]}-[m / 2] \leq s_{0}-[m / 2] \leq n_{0}-[m / 2] \leq N-[m / 2] .
$$

In other words, $s_{m}<0$ for $m>2 N+1$, which is impossible. Therefore, there exists $m_{0} \leq$ $2 N+1$ such that (12) holds. We put $r=r_{m_{0}}, \eta(d)=\frac{d}{(d+1) c^{2(N+1)}}, p=N$ and $k_{0}=s_{m_{0}}$. Then for all $\|J\| \neq k_{0}=s_{m_{0}}$ in $\mathbb{T}^{n}\left(z^{0}, \frac{r_{1}}{\mathbf{L}\left(z^{0}\right)}\right)$, in view (11) and (12) we obtain

$$
\left|b_{J}\right|\left|\left(z-z^{0}\right)^{J}\right|=a_{J}^{*} r^{\|J\|} \leq a_{\|J\|} r^{\|J\|} \leq \frac{1}{c} a_{s_{m_{0}}} r_{m_{0}}^{s_{m_{0}}}=\frac{1}{c} a_{k_{0}} r^{k_{0}} .
$$

Thus, for $z \in \mathbb{T}^{n}\left(z^{0}, \frac{r \nVdash}{\mathbf{L}\left(z^{0}\right)}\right)$

$$
\left|\sum_{\|J\| \neq k_{0}} b_{J}\left(z-z^{0}\right)^{J}\right| \leq \sum_{\|J\| \neq k_{0}} a_{j}^{*} r^{\|J\|} \leq \sum_{\substack{k=0, k \neq k_{0}}}^{\infty} a_{k} C_{n+k-1}^{k} r^{k}=
$$




$$
=\sum_{\substack{k=0, k \neq s_{m_{0}}}}^{s_{m_{0}-1}} a_{k} C_{n+k-1}^{k} r^{k}+\sum_{k=s_{m_{0}-1}+1}^{\infty} a_{k} C_{n+k-1}^{k} r^{k}
$$

We will estimate two sums in (15). From (12) it follows that $\mu_{m_{0}}^{*} \leq \frac{1}{c} \mu_{m_{0}}$ or $\max \left\{a_{k} r_{m_{0}}^{k}: k \neq\right.$ $\left.s_{m_{0}}, k \leq s_{m_{0}-1}\right\} \leq \frac{1}{c} \max \left\{a_{k} r_{m_{0}}^{k}: k \neq s_{m_{0}}, k \leq s_{m_{0}-1}\right\}$, i. e. $a_{k} r^{k} \leq \frac{1}{c} a_{k_{0}} r^{k_{0}}$. Taking into account (14), it can be deduced that

$$
\sum_{\substack{k=0, k \neq s_{m_{0}}}}^{s_{m_{0}-1}} a_{k} C_{n+k-1}^{k} r^{k} \leq \frac{a_{k_{0}} r^{k_{0}}}{c} \sum_{k=0}^{N} C_{n+k-1}^{k} \leq \frac{a_{k_{0}} r^{k_{0}}}{c}(N+1) C_{n+N-1}^{N} .
$$

For all $k \geq s_{m_{0}-1}+1 \quad a_{k} r_{m_{0}-1}^{k} \leq \mu_{m_{0}-1}$ holds. Then $a_{k} r_{m_{0}}^{k}=\frac{a_{k} r_{m_{0}-1}^{k}}{c^{k}} \leq \frac{\mu_{m_{0}-1}}{c^{k}}$. In view of (12) we deduce

$$
\begin{gathered}
\sum_{k=s_{m_{0}-1}+1}^{\infty} a_{k} C_{n+k-1}^{k} r^{k} \leq \mu_{m_{0}-1} \sum_{k=s_{m_{0}-1}+1}^{\infty} C_{n+k-1}^{k} \frac{1}{c^{k}} \leq \\
\leq a_{s_{m_{0}-1}} r_{m_{0}}^{s_{m_{0}-1}} c^{s_{m_{0}-1}} \sum_{k=s_{m_{0}-1}+1}(k+1)(k+2) \ldots(k+n) \frac{1}{c^{k}} \leq \\
\leq\left.\frac{a_{s_{m_{0}}} r^{s_{m_{0}}}}{c} c^{s_{m_{0}-1}}\left(\sum_{k=s_{m_{0}-1}+1}^{\infty} x^{k+n}\right)^{(n)}\right|_{x=\frac{1}{c}} ^{\infty}=\left.\frac{a_{k_{0}} r^{k_{0}}}{c} c^{s_{m_{0}-1}}\left\{\frac{x^{s_{m_{0}-1+n+1}}}{1-x}\right\}^{(n)}\right|_{x=\frac{1}{c}}= \\
=\frac{a_{k_{0}} r^{k_{0}}}{c} c^{s_{m_{0}-1}} \sum_{j=0}^{n} C_{n}^{j}(n-j) !\left(s_{m_{0}-1}+n+1\right) \ldots\left(s_{m_{0}-1}+n-j+2\right) \times \\
\times\left.\frac{x^{s_{m_{0}-1}+1+n-j}}{(1-x)^{n-j+1}}\right|_{x=\frac{1}{c}} \leq \frac{a_{k_{0}} r^{k_{0}}}{c} c^{s_{m_{0}}-1} n !(N+n+1) ! \sum_{j=0}^{n} \frac{(1 / c)^{s_{m_{0}-1}+1+n-j}}{(1-1 / c)^{n-j+1}}= \\
=n !(N+n+1) ! \frac{a_{k_{0}} r^{k_{0}}}{c} \sum_{j=0}^{n} \frac{1}{(c-1)^{n-j+1}} \leq(n+1) !(N+n+1) ! \frac{a_{k_{0}} r^{k_{0}}}{c}
\end{gathered}
$$

because $c \geq 2$. Hence, from (15)-(17) it follows that

$$
\left|\sum_{\|J\| \neq k_{0}} b_{J}\left(z-z^{0}\right)^{J}\right| \leq \frac{\left((N+1) C_{n+N-1}^{N}+(n+1) !(N+n+1) !\right) a_{k_{0}} r^{k_{0}}}{c} \leq \frac{1}{2} a_{k_{0}} r^{k_{0}} .
$$

It means that the polynomial $P_{k_{0}}$ is the dominating polynomial in the series $(10)$ on skeleton $\mathbb{T}^{n}\left(z^{0}, \frac{r \mathbf{1}}{\mathbf{L}\left(z^{0}\right)}\right)$.

Theorem 4. Let $\mathbf{L} \in Q\left(\mathbb{B}^{n}\right)$. If there exist $p \in \mathbb{Z}_{+}, d \in(0 ; 1], \eta \in(0 ; d)$ such that for each $z^{0} \in \mathbb{B}^{n}$ and some $R=\left(r_{1}, \ldots, r_{n}\right)$ with $r_{j}=r_{j}\left(d, z^{0}\right) \in(\eta, d), j \in\{1, \ldots, n\}$, and certain $k^{0}=k^{0}\left(d, z^{0}\right) \leq p$ the polynomial $p_{k^{0}}$ is the dominating polynomial in the series (10) on $\mathbb{T}^{2}\left(z^{0}, R / \mathbf{L}\left(z^{0}\right)\right)$ then the analytic in $\mathbb{B}^{n}$ function $F$ has bounded $\mathbf{L}$-index in joint variables.

Proof. Suppose that there exist $p \in \mathbb{Z}_{+}, d \leq 1$ and $\eta \in(0 ; d)$ such that for each $z^{0} \in \mathbb{B}^{n}$ and some $R=\left(r_{1}, \ldots, r_{n}\right)$ with $r_{j}=r_{j}\left(d, z^{0}\right) \in(\eta, d), j \in\{1, \ldots, n\}$, and $k_{0}=k_{0}\left(1, z^{0}\right) \leq p$ the 
polynomial $P_{k_{0}}$ is a dominating polynomial in the series $(10)$ on $\mathbb{T}^{n}\left(z^{0}, \frac{R}{\mathbf{L}\left(z^{0}\right)}\right)$. Let us denote $r_{0}=\max _{1 \leq j \leq n} r_{j}$. Then

$$
\left|\sum_{\|J\| \neq k_{0}} b_{J}\left(z-z^{0}\right)^{J}\right|=\left|F(z)-\sum_{\|J\|=k_{0}} b_{J}\left(z-z^{0}\right)^{J}\right| \leq \frac{a_{k_{0}} r_{0}^{k_{0}}}{2} .
$$

Using Cauchy's inequality we have $\left|b_{J}\left(z-z^{0}\right)^{J}\right|=a_{j}^{*} R^{J} \leq \frac{a_{k_{0}} r_{0}^{k_{0}}}{2}$ for all $J \in \mathbb{Z}_{+}^{n},\|J\| \neq k_{0}$, that is for all $\|J\|=k \neq k_{0}$

$$
a_{k} R^{J} \leq \frac{a_{k_{0}} r_{0}^{k_{0}}}{2}
$$

Suppose that $F$ is not a function of bounded $\mathbf{L}$-index in joint variables. Then in view of Theorem 1 for all $p_{1} \in \mathbb{Z}_{+}$and $c \geq 1$ there exists $z^{0} \in \mathbb{B}^{n}$ such that the next inequality

$$
\max \left\{\frac{\left|F^{(J)}\left(z^{0}\right)\right|}{\mathbf{L}^{J}\left(z^{0}\right)}:\|J\|=p_{1}+1\right\}>c \max \left\{\frac{\left|F^{(K)}\left(z^{0}\right)\right|}{\mathbf{L}^{K}\left(z^{0}\right)}:\|K\| \leq p_{1}\right\}
$$

holds. We put $p_{1}=p$ and $c=\left(\frac{(p+1) !}{\eta^{p+1}}\right)^{n}$. Then for this $z^{0}\left(p_{1}, c\right)$

$$
\max \left\{\frac{\left|F^{(J)}\left(z^{0}\right)\right|}{J ! \mathbf{L}^{J}\left(\left|z^{0}\right|\right)}:\|J\|=p+1\right\}>\frac{1}{\eta^{p+1}} \max \left\{\frac{\left|F^{(K)}\left(z^{0}\right)\right|}{K ! \mathbf{L}^{K}\left(\left|z^{0}\right|\right)}:\|K\| \leq p\right\}
$$

that is $a_{p+1}>\frac{a_{k_{0}}}{\eta^{p+1}}$. Hence, $a_{p+1} r_{0}^{p+1}>\frac{a_{k_{0}} r_{0}^{p+1}}{\eta^{p+1}} \geq a_{k_{0}} r^{k_{0}}$. The last inequality contradicts (18). Therefore, $F$ is of bounded $\mathbf{L}$-index in joint variables.

4. Properties of $Q\left(\mathbb{B}^{n}\right)$. Here we study some properties of the auxiliary class $Q\left(\mathbb{B}^{n}\right)$. Similar propositions for $\mathbb{C}^{n}$ are established in [15].

Theorem 5. Let $\mathbf{L}(z)=\left(l_{1}(z), \ldots, l_{n}(z)\right), l_{j}: \mathbb{B}^{n} \rightarrow \mathbb{C}$ and $\frac{\partial l_{j}}{\partial z_{m}}$ be continuous functions in $\mathbb{B}^{n}$, for all $j, m \in\{1,2, \ldots, n\}$. If for every $j \in\{1,2, \ldots, n\}\left|l_{j}(z)\right|$ satisfies (1) and there exist $P>0$ and $c>0$ such that for all $z \in \mathbb{B}^{n}$ and every $j, m \in\{1,2, \ldots, n\}$

$$
\frac{1}{c+\left|l_{j}(z)\right|}\left|\frac{\partial l_{j}(z)}{\partial z_{m}}\right| \leq P
$$

then $\mathbf{L}^{*} \in Q\left(\mathbb{B}^{n}\right)$, where $\mathbf{L}^{*}(z)=\left(c+\left|l_{1}(z)\right|, \ldots, c+\left|l_{n}(z)\right|\right)$.

Proof. Clearly, the function $\mathbf{L}^{*}(z)$ is positive and continuous. For given $z \in \mathbb{B}^{n}, z^{0} \in \mathbb{B}^{n}$ we define an analytic curve $\varphi:[0,1] \rightarrow \mathbb{B}^{n}$

$$
\varphi_{j}(\tau)=z_{j}^{0}+\tau\left(z_{j}-z_{j}^{0}\right), j \in\{1,2, \ldots, n\},
$$

where $\tau \in[0,1]$. It is known that for every continuously differentiable function $g$ of real variable $\tau$ the inequality $\frac{d}{d t}|g(\tau)| \leq\left|g^{\prime}(\tau)\right|$ holds except the points where $g(\tau)=0$. Using assymptions of this lemma, we establish the upper estimate of $\lambda_{2, j}\left(z_{0}, R\right)$ :

$$
\lambda_{2, j}\left(z_{0}, R\right)=\sup \left\{\frac{c+\left|l_{j}(z)\right|}{c+\left|l_{j}\left(z^{0}\right)\right|}: z \in \mathbb{D}^{n}\left[z^{0}, \frac{R}{\mathbf{L}^{*}\left(z^{0}\right)}\right]\right\}=
$$




$$
\begin{aligned}
& =\sup _{z \in \mathbb{D}^{n}\left[z^{0}, \overline{\mathbf{L}^{*}\left(z^{0}\right)}\right]}\left\{\exp \left\{\ln \left(c+\left|l_{j}(z)\right|\right)-\ln \left(c+\left|l_{j}\left(z^{0}\right)\right|\right)\right\}=\right. \\
& =\sup \left\{\exp \left\{\int_{0}^{1} \frac{d\left(c+\left|l_{j}(\varphi(\tau))\right|\right)}{c+\left|l_{j}(\varphi(\tau))\right|}\right\}: z \in \mathbb{D}^{n}\left[z^{0}, \frac{R}{\mathbf{L}^{*}\left(z^{0}\right)}\right]\right\} \leq \\
& \leq \sup _{z \in \mathbb{D}^{n}\left[z^{0}, \frac{R}{\mathbf{L}^{*}\left(z^{0}\right)}\right]}\left\{\exp \left\{\int_{0}^{1} \sum_{m=1}^{n} \frac{\left|\varphi_{m}^{\prime}(\tau)\right|}{c+\left|l_{j}(\varphi(\tau))\right|}\left|\frac{\partial l_{j}(\varphi(\tau))}{\partial z_{m}}\right| d \tau\right\}\right\} \leq \\
& \leq \sup _{z \in \mathbb{D}^{n}\left[z^{0}, \frac{R}{\mathbf{L}^{*}\left(z^{0}\right)}\right]}\left\{\exp \left\{\int_{0}^{1} \sum_{m=1}^{n} P\left|z_{m}-z_{m}^{0}\right| d \tau\right\}\right\} \leq \\
& \leq \sup _{z \in \mathbb{D}^{n}\left[z^{0}, \frac{R}{\mathbf{L}^{*}\left(z^{0}\right)}\right]}\left\{\exp \left\{\sum_{m=1}^{n} \frac{P r_{j}}{c+\left|l_{m}\left(z^{0}\right)\right|}\right\}\right\} \leq \exp \left(\frac{P}{c} \sum_{m=1}^{n} r_{j}\right) .
\end{aligned}
$$

Hence, for all

$$
R \geq 0 \quad \lambda_{2, j}(R)=\sup _{z^{0} \in \mathbb{B}^{n}} \lambda_{2, j}\left(z^{0}, \eta\right) \leq \exp \left(\frac{P}{c} \sum_{m=1}^{n} r_{j}\right)<\infty .
$$

Using the inequality $\frac{d}{d t}|g(t)| \geq-\left|g^{\prime}(t)\right|$ it can be proved that for every $\eta \geq 0$ one has $\lambda_{1, j}(R) \geq \exp \left(-\frac{P}{c} \sum_{m=1}^{n} r_{j}\right)>0$. Therefore, $\mathbf{L}^{*} \in Q\left(\mathbb{B}^{n}\right)$.

Particularly, if $\mathbf{L}(z)=\left(l_{1}(R), \ldots, l_{n}(R)\right), R=\left(\left|z_{1}\right|, \ldots,\left|z_{n}\right|\right)$, for every $j \in\{1, \ldots, n\}$ the function $l_{j}(R)$ is positive continuously differentiable and $\left|\nabla \ln l_{j}(R)\right| \leq P$ for all $|R|<1$ then $\mathbf{L} \in Q\left(\mathbb{B}^{n}\right)$, where

$$
\nabla l_{j}(R)=\left(\frac{\partial l_{j}(R)}{\partial r_{1}}, \ldots, \frac{\partial l_{j}(R)}{r_{n}}\right) .
$$

At first we prove the following lemma.

Lemma 1. If $\mathbf{L} \in Q\left(\mathbb{B}^{n}\right)$ then for every $j \in\{1, \ldots, n\}$ and for every fixed $z^{*} \in \mathbb{B}^{n}\left|z_{j}\right| l_{j}\left(z^{*}+\right.$ $\left.z_{j} \mathbf{1}_{j}\right) \rightarrow \infty$ as $\left|z^{*}+z_{j} \mathbf{1}_{j}\right| \rightarrow 1-0$.

Proof. In view of (1) we have $l_{j}\left(z^{*}+z_{j} \mathbf{1}_{j}\right) \geq \frac{\beta}{1-\left|z^{*}+z_{j} \mathbf{1}_{j}\right|} \rightarrow+\infty$ as $\left|z^{*}+z_{j} \mathbf{1}_{j}\right| \rightarrow 1-0$.

5. Estimates of growth of analytic functions in ball. Denote

$$
[0,2 \pi]^{n}=\underbrace{[0,2 \pi] \times \cdots \times[0,2 \pi]}_{n-\text { th times }} .
$$

For $R=\left(r_{1}, \ldots, r_{n}\right) \in \mathbb{R}_{+}^{n}, \Theta=\left(\theta_{1}, \ldots, \theta_{n}\right) \in[0,2 \pi]^{n}, \quad A=\left(a_{1}, \ldots, a_{n}\right) \in \mathbb{C}^{n}$ we write

$$
R e^{i \Theta}=\left(r_{1} e^{i \theta_{1}}, \ldots, r_{n} e^{i \theta_{n}}\right), \arg A=\left(\arg a_{1}, \ldots, \arg a_{n}\right) .
$$

By $K\left(\mathbb{B}^{n}\right)$ we denote the class of positive continuous functions $\mathbf{L}=\left(l_{1}, \ldots, l_{n}\right)$, where $l_{j}: \mathbb{B}^{n} \rightarrow \mathbb{R}_{+}$satisfy (1) and there exists $c \geq 1$ such that for every $R \in \mathbb{R}_{+}^{n}$ with $|R|<1$ and $j \in\{1, \ldots, n\}$

$$
\max _{\Theta_{1}, \Theta_{2} \in[0,2 \pi]^{n}} \frac{l_{j}\left(R e^{i \Theta_{2}}\right)}{l_{j}\left(R e^{i \Theta_{1}}\right)} \leq c .
$$


If $\mathbf{L}(z)=\left(l_{1}\left(\left|z_{1}\right|, \ldots,\left|z_{n}\right|\right), \ldots, l_{n}\left(\left|z_{1}\right|, \ldots,\left|z_{n}\right|\right)\right)$ then $\mathbf{L} \in K\left(\mathbb{B}^{n}\right)$. It is easy to prove that $\frac{\left|e^{z}\right|+1}{1-|z|} \in Q(\mathbb{D}) \backslash K(\mathbb{D})$, but $\frac{e^{e^{|z|}}}{1-|z|} \in K(\mathbb{D}) \backslash Q(\mathbb{D})$. Besides, if $\mathbf{L}_{1}, \mathbf{L}_{2} \in K\left(\mathbb{B}^{n}\right)$ then $\mathbf{L}_{1}+\mathbf{L}_{2} \in$ $K\left(\mathbb{B}^{n}\right)$ and $\mathbf{L}_{1} \mathbf{L}_{2} \in K\left(\mathbb{B}^{n}\right)$. For simplicity, let us to write $M(F, R)=\max \{|F(z)|: z \in$ $\left.\mathbb{T}^{n}(\mathbf{0}, R)\right\}$, where $|R|<1$. Denote $\boldsymbol{\beta}=\left(\frac{\beta}{c \sqrt{n}}, \ldots, \frac{\beta}{c \sqrt{n}}\right)$.

Theorem 6. Let $\mathbf{L} \in Q\left(\mathbb{B}^{n}\right) \cap K\left(\mathbb{B}^{n}\right), \beta>c \sqrt{n}$. If an analytic function $F$ in $\mathbb{B}^{n}$ has bounded L-index in joint variables, then

$$
\ln M(F, R)=O\left(\min _{\sigma_{n} \in \mathcal{S}_{n}} \min _{\Theta \in[0,2 \pi]^{n}} \sum_{j=1}^{n} \int_{0}^{r_{j}} l_{j}\left(R\left(j, \sigma_{n}, t\right) e^{i \Theta}\right) d t\right) \text { as }|R| \rightarrow 1-0,
$$

where $\sigma_{n}$ is a permutation of $\{1, \ldots, n\}, \mathcal{S}_{n}$ is a set of all permutations of $\{1, \ldots, n\}$,

$$
R\left(j, \sigma_{n}, t\right)=\left(r_{1}^{\prime}, \ldots, r_{n}^{\prime}\right), \quad r_{k}^{\prime}=\left\{\begin{array}{l}
r_{k}^{0}, \text { if } \sigma_{n}(k)<j, \\
t, \text { if } k=j, \\
r_{k}, \text { if } \sigma_{n}(k)>j
\end{array} \quad k \in\{1, \ldots, n\}\right.
$$

$R^{0}=\left(r_{1}^{0}, \ldots, r_{n}^{0}\right)$ is a fixed radius.

Proof. Let $R>\mathbf{0},|R|<1, \Theta \in[0,2 \pi]^{n}$ and the point $z^{*} \in \mathbb{T}^{n}\left(\mathbf{0}, R+\frac{\boldsymbol{\beta}}{\mathbf{L}\left(R e^{i \Theta}\right)}\right)$ be a such that

$$
\left|F\left(z^{*}\right)\right|=\max \left\{|F(z)|: z \in \mathbb{T}^{n}\left(\mathbf{0}, R+\frac{\boldsymbol{\beta}}{\mathbf{L}\left(R e^{i \Theta}\right)}\right)\right\} .
$$

Denote $z^{0}=\frac{z^{*} R}{R+\boldsymbol{\beta} / \mathbf{L}\left(R e^{i \Theta}\right)}$. Then

$$
\begin{gathered}
\left|z_{j}^{0}-z_{j}^{*}\right|=\left|\frac{z_{j}^{*} r_{j}}{r_{j}+\frac{\beta}{c \sqrt{n} l_{j}\left(R e^{i \Theta}\right)}}-z_{j}^{*}\right|=\left|\frac{z_{j}^{*} \beta /\left(c \sqrt{n} l_{j}\left(R e^{i \Theta}\right)\right)}{r_{j}+\frac{\beta}{c \sqrt{n} l_{j}\left(R e^{i \Theta}\right)} \mid}\right|=\frac{\beta}{c \sqrt{n} l_{j}\left(R e^{i \Theta}\right)}, \\
\mathbf{L}\left(z^{0}\right)=\mathbf{L}\left(\frac{z^{*} R}{R+\boldsymbol{\beta} / \mathbf{L}\left(R e^{i \Theta}\right)}\right)=\mathbf{L}\left(\frac{\left(R+\boldsymbol{\beta} / \mathbf{L}\left(R e^{i \Theta}\right)\right) e^{i \arg z^{*}} R}{R+\boldsymbol{\beta} / \mathbf{L}\left(R e^{i \Theta}\right)}\right)=\mathbf{L}\left(R e^{i \arg z^{*}}\right) .
\end{gathered}
$$

Since $\mathbf{L} \in K\left(\mathbb{B}^{n}\right)$ we have that $c \mathbf{L}\left(z^{0}\right)=c \mathbf{L}\left(R e^{i \arg z^{*}}\right) \geq \mathbf{L}\left(R e^{i \Theta}\right) \geq \frac{1}{c} \mathbf{L}\left(z^{0}\right)$. We consider two skeletons $\mathbb{T}^{n}\left(z^{0}, \frac{1}{\mathbf{L}\left(z^{0}\right)}\right)$ and $\mathbb{T}^{n}\left(z^{0}, \frac{\boldsymbol{\beta}}{\mathbf{L}\left(z^{0}\right)}\right)$. By Theorem 2 there exists $p_{1}=p_{1}\left(\frac{\mathbf{1}}{c}, c \boldsymbol{\beta}\right) \geq 1$ such that (9) holds with $R^{\prime}=\frac{1}{c}, R^{\prime \prime}=c \boldsymbol{\beta}$, i.e.

$$
\begin{gathered}
\max \left\{|F(z)|: z \in \mathbb{T}^{n}\left(\mathbf{0}, R+\frac{\boldsymbol{\beta}}{\mathbf{L}\left(R e^{i \Theta}\right)}\right)\right\}=\left|F\left(z^{*}\right)\right| \leq \\
\leq \max \left\{|F(z)|: z \in \mathbb{T}^{n}\left(z^{0}, \frac{\boldsymbol{\beta}}{\mathbf{L}\left(R e^{i \Theta}\right)}\right)\right\} \leq \max \left\{|F(z)|: z \in \mathbb{T}^{n}\left(z^{0}, \frac{c \boldsymbol{\beta}}{\mathbf{L}\left(z^{0}\right)}\right)\right\} \leq \\
\leq p_{1} \max \left\{|F(z)|: z \in \mathbb{T}^{n}\left(z^{0}, \frac{\mathbf{1}}{c \mathbf{L}\left(z^{0}\right)}\right)\right\} \leq p_{1} \max \left\{|F(z)|: z \in \mathbb{T}^{n}\left(\mathbf{0}, R+\frac{\mathbf{1}}{\mathbf{L}\left(R e^{i \Theta}\right)}\right)\right\} .
\end{gathered}
$$

The function $\ln ^{+} \max \left\{|F(z)|: z \in \mathbb{T}^{n}(\mathbf{0}, R)\right\}$ is a convex function of the variables $\ln r_{1}, \ldots$, $\ln r_{n}$ (see [30, p. 84]). Hence, the function admits a representation

$$
\ln ^{+} \max \left\{|F(z)|: z \in \mathbb{T}^{n}(\mathbf{0}, R)\right\}-\ln ^{+} \max \left\{|F(z)|: z \in \mathbb{T}^{n}\left(\mathbf{0}, R+\left(r_{j}^{0}-r_{j}\right) \mathbf{1}_{j}\right)\right\}=
$$




$$
=\int_{r_{j}^{0}}^{r_{j}} \frac{A_{j}\left(r_{1}, \ldots, r_{j-1}, t, r_{j+1}, \ldots, r_{n}\right)}{t} d t
$$

for arbitrary $0<r_{j}^{0} \leq r_{j}$, where the functions $A_{j}\left(r_{1}, \ldots, r_{j-1}, t, r_{j+1}, \ldots, r_{n}\right)$ are positive non-decreasing in variable $t, j \in\{1, \ldots, n\}$.

Using (21) we deduce

$$
\begin{gathered}
\ln p_{1} \geq \ln \max \left\{|F(z)|: z \in \mathbb{T}^{n}\left(\mathbf{0}, R+\frac{\boldsymbol{\beta}}{\mathbf{L}\left(R e^{i \Theta}\right)}\right)\right\}- \\
-\ln \max \left\{|F(z)|: z \in \mathbb{T}^{n}\left(\mathbf{0}, R+\frac{\mathbf{1}}{\mathbf{L}\left(R e^{i \Theta}\right)}\right)\right\}= \\
=\sum_{j=1}^{n} \ln \max \left\{|F(z)|: z \in \mathbb{T}^{n}\left(\mathbf{0}, R+\frac{\mathbf{1}+\sum_{k=j}^{n}\left(\frac{\beta}{c \sqrt{n}}-1\right) \mathbf{1}_{k}}{\mathbf{L}\left(R e^{i \Theta}\right)}\right)\right\}- \\
-\ln \max \left\{|F(z)|: z \in \mathbb{T}^{n}\left(\mathbf{0}, R+\frac{\mathbf{1}+\sum_{k=j+1}^{n}\left(\frac{\beta}{c \sqrt{n}}-1\right) \mathbf{1}_{k}}{\mathbf{L}\left(R e^{i \Theta}\right)}\right)\right\}= \\
=\sum_{j=1}^{n} \int_{r_{j}+1 / l_{j}\left(R e^{i \Theta}\right)}^{r_{j}+\beta /\left(c \sqrt{n} l_{j}\left(R e^{i \Theta}\right)\right)}{ }^{\prime} A_{j}\left(r_{1}+\frac{1}{l_{1}\left(R e^{i \Theta}\right)}, \ldots, r_{j-1}+\frac{1}{l_{j-1}\left(R e^{i \Theta}\right)}, t\right. \\
\left.r_{j+1}+\frac{\beta}{c \sqrt{n} l_{j+1}\left(R e^{i \Theta}\right)}, \ldots, r_{n}+\frac{\beta}{c \sqrt{n} l_{n}\left(R e^{i \Theta}\right)}\right) d t \geq \\
\frac{\beta}{c \sqrt{n}}-1 \\
\sum_{j=1}^{n} \ln \left(1+\frac{1}{r_{j} l_{j}\left(R e^{i \Theta}\right)+1}\right) A_{j}\left(r_{1}+\frac{1}{l_{1}\left(R e^{i \Theta}\right)}, \ldots, r_{j-1}+\frac{1}{l_{j-1}\left(R e^{i \Theta}\right)}, r_{j},\right. \\
\left.r_{j+1}+\frac{\beta}{c \sqrt{n} l_{j+1}\left(R e^{i \Theta}\right)}, \ldots, r_{n}+\frac{\beta}{c \sqrt{n} l_{n}\left(R e^{i \Theta}\right)}\right) .
\end{gathered}
$$

By Lemma 1 the function $r_{j} l_{j}\left(R e^{i \Theta}\right) \rightarrow+\infty$ as $|R| \rightarrow 1-0$. Hence, for $j \in\{1, \ldots, n\}$ and $r_{i} \geq r_{i}^{0}$

$$
\ln \left(1+\frac{\frac{\beta}{c \sqrt{n}}-1}{r_{j} l_{j}\left(R e^{i \Theta}\right)+1}\right) \sim \frac{\frac{\beta}{c \sqrt{n}}-1}{r_{j} l_{j}\left(R e^{i \Theta}\right)+1} \geq \frac{\frac{\beta}{c \sqrt{n}}-1}{2 r_{j} l_{j}\left(R e^{i \Theta}\right)},|R| \rightarrow 1-0 .
$$

Thus, for every $j \in\{1, \ldots, n\}$ inequality (23) implies that

$$
\begin{gathered}
A_{j}\left(r_{1}+\frac{1}{l_{1}\left(R e^{i \Theta}\right)}, \ldots, r_{j-1}+\frac{1}{l_{j-1}\left(R e^{i \Theta}\right)}, r_{j}, r_{j+1}+\frac{\beta}{c \sqrt{n} l_{i+1}\left(R e^{i \Theta}\right)}, \ldots,\right. \\
\left.r_{n}+\frac{\beta}{c \sqrt{n} l_{n}\left(R e^{i \Theta}\right)}\right) \leq \frac{2 \ln p_{1}}{\frac{\beta}{c \sqrt{n}}-1} r_{j} l_{j}\left(R e^{i \Theta}\right) .
\end{gathered}
$$

Let $R^{0}=\left(r_{1}^{0}, \ldots, r_{n}^{0}\right)$, where every $r_{j}^{0}$ is chosen above. Applying $(22) n$-th times we obtain consequently

$$
\ln \max \left\{|F(z)|: z \in \mathbb{T}^{n}(\mathbf{0}, R)\right\}=
$$




$$
\begin{gathered}
=\ln \max \left\{|F(z)|: z \in \mathbb{T}^{n}\left(\mathbf{0}, R+\left(r_{1}^{0}-r_{1}\right) \mathbf{1}_{1}\right)\right\}+\int_{r_{1}^{0}}^{r_{1}} \frac{A_{1}\left(t, r_{2}, \ldots, r_{n}\right)}{t} d t= \\
=\ln \max \left\{|F(z)|: z \in \mathbb{T}^{n}\left(\mathbf{0}, R+\left(r_{1}^{0}-r_{1}\right) \mathbf{1}_{1}+\left(r_{2}^{0}-r_{2}\right) \mathbf{1}_{2}\right)\right\}+ \\
\quad+\int_{r_{1}^{0}}^{r_{1}} \frac{A_{1}\left(t, r_{2}, \ldots, r_{n}\right)}{t} d t+\int_{r_{2}^{0}}^{r_{2}} \frac{A_{2}\left(r_{1}^{0}, t, r_{3} \ldots, r_{n}\right)}{t} d t= \\
=\ln \max \left\{|F(z)|: z \in \mathbb{T}^{n}\left(\mathbf{0}, R^{0}\right)\right\}+\sum_{j=1}^{n} \int_{r_{j}^{0}}^{r_{j}} \frac{A_{j}\left(r_{1}^{0}, \ldots, r_{j-1}^{0}, t, r_{j+1}, \ldots, r_{n}\right)}{t} d t \leq \\
\quad \leq \frac{\ln \max \left\{|F(z)|: z \in \mathbb{T}^{n}\left(\mathbf{0}, R^{0}\right)\right\}+}{\frac{\beta}{c \sqrt{n}}-1} \sum_{j=1}^{n} \int_{r_{j}^{0}}^{r_{j}} l_{j}\left(r_{1}^{0} e^{i \theta_{1}}, \ldots, r_{j-1}^{0} e^{i \theta_{j-1}}, t e^{i \theta_{j}}, r_{j+1} e^{i \theta_{j+1}}, \ldots, r_{n} e^{i \theta_{n}}\right) d t \leq \\
\quad \leq \frac{\ln \max \left\{|F(z)|: z \in \mathbb{T}^{n}\left(\mathbf{0}, R^{0}\right)\right\}+}{\frac{\beta}{c \sqrt{n}}-1} \sum_{j=1}^{n} \int_{0}^{r_{j}} l_{j}\left(r_{1}^{0} e^{i \theta_{1}}, \ldots, r_{j-1}^{0} e^{i \theta_{j-1}}, t e^{i \theta_{j}}, r_{j+1} e^{i \theta_{j+1}}, \ldots, r_{n} e^{i \theta_{n}}\right) d t \leq \\
\leq(1+o(1)) \frac{2 \ln p_{1}}{\frac{\beta}{c \sqrt{n}}-1} \sum_{j=1}^{n} \int_{0}^{r_{j}} l_{j}\left(r_{1}^{0} e^{i \theta_{1}}, \ldots, r_{j-1}^{0} e^{i \theta_{j-1}}, t e^{i \theta_{j}}, r_{j+1} e^{i \theta_{j+1}}, \ldots, r_{n} e^{i \theta_{n}}\right) d t .
\end{gathered}
$$

The function $\ln \max \left\{|F(z)|: z \in \mathbb{T}^{n}(\mathbf{0}, R)\right\}$ is independent of $\Theta$. Thus, the following estimate

$$
\begin{gathered}
\ln \max \left\{|F(z)|: z \in \mathbb{T}^{n}(\mathbf{0}, R)\right\}= \\
=O\left(\min _{\Theta \in[0,2 \pi]^{n}} \sum_{j=1}^{n} \int_{0}^{r_{j}} l_{j}\left(r_{1}^{0} e^{i \theta_{1}}, \ldots, r_{j-1}^{0} e^{i \theta_{j-1}}, t e^{i \theta_{j}}, r_{j+1} e^{i \theta_{j+1}}, \ldots, r_{n} e^{i \theta_{n}}\right) d t\right),
\end{gathered}
$$

holds as $|R| \rightarrow 1-0$. Obviously, the similar equality can be proved for arbitrary permutation $\sigma_{n}$ of the set $\{1,2, \ldots, n\}$. Thus, estimate (20) holds. Theorem 6 is proved.

Corollary 1. If $\mathbf{L} \in Q\left(\mathbb{B}^{n}\right) \cap K\left(\mathbb{B}^{n}\right), \min _{\Theta \in[0,2 \pi]^{n}} l_{j}\left(R e^{i \Theta}\right)$ is non-decreasing in each variable $r_{k}$, $k, j \in\{1, \ldots, n\}, k \neq j$, analytic function $F$ in $\mathbb{B}^{n}$ has bounded $\mathbf{L}$-index in joint variables then

$$
\ln \max \left\{|F(z)|: z \in \mathbb{T}^{n}(\mathbf{0}, R)\right\}=O\left(\min _{\Theta \in[0,2 \pi]^{n}} \sum_{j=1}^{n} \int_{0}^{r_{j}} l_{j}\left(R^{(j)} e^{i \Theta}\right) d t\right)
$$

as $|R| \rightarrow 1-0$, where $R^{(j)}=\left(r_{1}, \ldots, r_{j-1}, t, r_{j+1}, \ldots, r_{n}\right)$.

Note that Theorem 6 is new even for $n=1$ (see Theorem 3.3 in [35]) because we replace the condition $l=l(|z|)$ by the condition $l \in K(\mathbb{D})$, i.e. there exists $c>0$ such that for every $r \in(0,1) \max _{\theta_{1}, \theta_{2} \in[0,2 \pi]} \frac{l\left(r e^{i \theta_{2}}\right)}{l\left(r e^{i \theta_{1}}\right)} \leq c$. Particularly, the following proposition is valid.

Corollary 2. If $l \in Q \cap K$ and an analytic function $f$ in $\mathbb{D}$ has bounded l-index then

$$
\ln \max \{|f(z)|:|z|=r\}=O\left(\min _{\theta \in[0,2 \pi]} \int_{0}^{r} l\left(t e^{i \Theta}\right) d t\right) \quad \text { as } r \rightarrow 1-0 .
$$

Let us denote $a^{+}=\max \{a, 0\}, u_{j}(t)=u_{j}(t, R, \Theta)=l_{j}\left(\frac{t R}{r^{*}} e^{i \Theta}\right)$, where $a \in \mathbb{R}, t \in \mathbb{R}_{+}$, $j \in\{1, \ldots, n\}, r^{*}=\max _{1 \leq j \leq n} r_{j} \neq 0$ and $\frac{t}{r^{*}}|R|<1$. 
Theorem 7. Let $\mathbf{L}\left(R e^{i \Theta}\right)$ be a positive continuously differentiable function in each variable $r_{k}, k \in\{1, \ldots, n\},|R|<1, \Theta \in[0,2 \pi]^{n}$. If the function $\mathbf{L}$ satisfies (1) and an analytic function $F$ in $\mathbb{B}^{n}$ has bounded $\mathbf{L}$-index $N=N(F, \mathbf{L})$ in joint variables then for every $\Theta \in$ $[0,2 \pi]^{n}$ and for every $R \in \mathbb{R}_{+}^{n},|R|<1$, and $S \in \mathbb{Z}_{+}^{n}$

$$
\begin{gathered}
\ln \max \left\{\frac{\left|F^{(S)}\left(R e^{i \Theta}\right)\right|}{S ! \mathbf{L}^{S}\left(R e^{i \Theta}\right)}:\|S\| \leq N\right\} \leq \ln \max \left\{\frac{\left|F^{(S)}(\mathbf{0})\right|}{S ! \mathbf{L}^{S}(\mathbf{0})}:\|S\| \leq N\right\}+ \\
+\int_{0}^{r^{*}}\left(\max _{\|S\| \leq N}\left\{\sum_{j=1}^{n} \frac{r_{j}}{r^{*}}\left(k_{j}+1\right) l_{j}\left(\frac{\tau}{r^{*}} R e^{i \Theta}\right)\right\}+\max _{\|S\| \leq N}\left\{\sum_{j=1}^{n} \frac{k_{j}\left(-u_{j}^{\prime}(\tau)\right)^{+}}{l_{j}\left(\frac{\tau}{r^{*}} R e^{i \Theta}\right)}\right\}\right) d \tau .
\end{gathered}
$$

Proof. Let $R \in \mathbb{R} \backslash\{\mathbf{0}\}, \Theta \in[0,2 \pi]^{n}$. Denote $\alpha_{j}=\frac{r_{j}}{r^{*}}, j \in\{1, \ldots, n\}$ and $A=\left(\alpha_{1}, \ldots, \alpha_{n}\right)$. We consider the function

$$
g(t)=\max \left\{\frac{\left|F^{(S)}\left(A t e^{i \Theta}\right)\right|}{S ! \mathbf{L}^{S}\left(A t e^{i \Theta}\right)}:\|S\| \leq N\right\},
$$

where $A t=\left(\alpha_{1} t, \ldots, \alpha_{n} t\right), A t e^{i \Theta}=\left(\alpha_{1} t e^{i \theta_{1}}, \ldots, \alpha_{n} t e^{i \theta_{n}}\right)$.

Since the function $\frac{\left|F^{(S)}\left(A t e^{i \Theta}\right)\right|}{K ! \mathbf{L}^{K}\left(A t e^{i \Theta}\right)}$ is continuously differentiable by real $t \in[0,+\infty)$, outside the zero set of the function $\left|F^{(S)}\left(A t e^{i \Theta}\right)\right|$, the function $g(t)$ is a continuously differentiable function on $\left[0, \frac{r^{*}}{|R|}\right)$, except, perhaps, for a countable set of points.

Therefore, using the inequality $\frac{d}{d r}|g(r)| \leq\left|g^{\prime}(r)\right|$ which holds except for the points $r=t$ such that $g(t)=0$, we deduce

$$
\begin{gathered}
\frac{d}{d t}\left(\frac{\left|F^{(S)}\left(A t e^{i \Theta}\right)\right|}{S ! \mathbf{L}^{S}\left(A t e^{i \Theta}\right)}\right)=\frac{1}{S ! \mathbf{L}^{S}\left(A t e^{i \Theta}\right)} \frac{d}{d t}\left|F^{(S)}\left(A t e^{i \Theta}\right)\right|+ \\
+\left|F^{(S)}\left(A t e^{i \Theta}\right)\right| \frac{d}{d t} \frac{1}{S ! \mathbf{L}^{S}\left(A t e^{i \Theta}\right)} \leq \frac{1}{S ! \mathbf{L}^{S}\left(A t e^{i \Theta}\right)}\left|\sum_{j=1}^{n} F^{\left(S+\mathbf{1}_{j}\right)}\left(A t e^{i \Theta}\right) \alpha_{j} e^{i \theta_{j}}\right|- \\
-\frac{\left|F^{(S)}\left(A t e^{i \Theta}\right)\right|}{S ! \mathbf{L}^{S}\left(A t e^{i \Theta}\right)} \sum_{j=1}^{n} \frac{k_{j} u_{j}^{\prime}(t)}{l_{j}\left(A t e^{i \Theta}\right)} \leq \sum_{j=1}^{n} \frac{\left|F^{\left(S+\mathbf{1}_{j}\right)}\left(A t e^{i \Theta}\right)\right|}{\left(S+\mathbf{1}_{j}\right) ! \mathbf{L}^{S+\mathbf{1}_{j}}\left(A t e^{i \Theta}\right)} \alpha_{j}\left(k_{j}+1\right) l_{j}\left(A t e^{i \Theta}\right)+ \\
+\frac{\left|F^{(S)}\left(A t e^{i \Theta}\right)\right|}{S ! \mathbf{L}^{S}\left(A t e^{i \Theta}\right)} \sum_{j=1}^{n} \frac{k_{j}\left(-u_{j}^{\prime}(t)\right)^{+}}{l_{j}\left(A t e^{i \Theta}\right)} .
\end{gathered}
$$

For absolutely continuous functions $h_{1}, h_{2}, \ldots, h_{k}$ and $h(x):=\max \left\{h_{j}(z): 1 \leq j \leq k\right\}$, $h^{\prime}(x) \leq \max \left\{h_{j}^{\prime}(x): 1 \leq j \leq k\right\}, x \in[a, b]$ (see [35, Lemma 4.1, p. 81]). The function $g$ is absolutely continuous, therefore, from (26) it follows that

$$
\begin{gathered}
g^{\prime}(t) \leq \max \left\{\frac{d}{d t}\left(\frac{\left|F^{(S)}\left(A t e^{i \Theta}\right)\right|}{S ! \mathbf{L}^{S}\left(A t e^{i \Theta}\right)}\right):\|S\| \leq N\right\} \leq \\
\leq \max _{\|S\| \leq N}\left\{\sum_{j=1}^{n} \frac{\alpha_{j}\left(s_{j}+1\right) l_{j}\left(A t e^{i \Theta}\right)\left|F^{\left(S+\mathbf{1}_{j}\right)}\left(A t e^{i \Theta}\right)\right|}{\left(K+\mathbf{1}_{j}\right) ! \mathbf{L}^{K+\mathbf{1}_{j}}\left(A t e^{i \Theta}\right)}+\frac{\left|F^{(S)}\left(A t e^{i \Theta}\right)\right|}{S ! \mathbf{L}^{S}\left(A t e^{i \Theta}\right)} \sum_{j=1}^{n} \frac{s_{j}\left(-u_{j}^{\prime}(t)\right)^{+}}{l_{j}\left(A t e^{i \Theta}\right)}\right\} \leq \\
\leq g(t)\left(\max _{\|S\| \leq N}\left\{\sum_{j=1}^{n} \alpha_{j}\left(s_{j}+1\right) l_{j}\left(A t e^{i \Theta}\right)\right\}+\max _{\|S\| \leq N}\left\{\sum_{j=1}^{n} \frac{s_{j}\left(-u_{j}^{\prime}(t)\right)^{+}}{l_{j}\left(A t e^{i \Theta}\right)}\right\}\right)=g(t)(\beta(t)+\gamma(t)),
\end{gathered}
$$


where

$$
\beta(t)=\max _{\|S\| \leq N}\left\{\sum_{j=1}^{n} \alpha_{j}\left(s_{j}+1\right) l_{j}\left(A t e^{i \Theta}\right)\right\}, \gamma(t)=\max _{\|S\| \leq N}\left\{\sum_{j=1}^{n} \frac{s_{j}\left(-u_{j}^{\prime}(t)\right)^{+}}{l_{j}\left(A t e^{i \Theta}\right)}\right\} .
$$

Thus, $\frac{d}{d t} \ln g(t) \leq \beta(t)+\gamma(t)$ and

$$
g(t) \leq g(0) \exp \int_{0}^{t}(\beta(\tau)+\gamma(\tau)) d \tau,
$$

because $g(0) \neq 0$. But $r^{*} A=R$. Substituting $t=r^{*}$ in (27) and taking into account (25), we deduce

$$
\begin{aligned}
& \ln \max \left\{\frac{\left|F^{(S)}\left(R e^{i \Theta}\right)\right|}{S ! \mathbf{L}^{S}\left(R e^{i \Theta}\right)}:\|S\| \leq N\right\} \leq \ln \max \left\{\frac{\left|F^{(S)}(\mathbf{0})\right|}{S ! \mathbf{L}^{S}(\mathbf{0})}:\|S\| \leq N\right\}+ \\
& +\int_{0}^{r^{*}}\left(\max _{\|S\| \leq N}\left\{\sum_{j=1}^{n} \alpha_{j}\left(s_{j}+1\right) l_{j}\left(A \tau e^{i \Theta}\right)\right\}+\max _{\|S\| \leq N}\left\{\sum_{j=1}^{n} \frac{s_{j}\left(-u_{j}^{\prime}(\tau)\right)^{+}}{l_{j}\left(A \tau e^{i \Theta}\right)}\right\}\right) d \tau,
\end{aligned}
$$

i.e. (24) is proved.

Theorem 8. Let $\mathbf{L}\left(R e^{i \Theta}\right)$ be a positive continuously differentiable function in each variable $r_{k}, k \in\{1, \ldots, n\},|R|<1, \Theta \in[0,2 \pi]^{n}$. If the function $\mathbf{L}$ satisfies (1) and an analytic function $F$ in $\mathbb{B}^{n}$ has bounded $\mathbf{L}$-index $N=N(F, \mathbf{L})$ in joint variables and there exists $C>0$ such that the function $\mathbf{L}$ satisfies inequalities

$$
\sup _{|R|<1} \max _{t \in\left[0, r^{*}\right]} \max _{\Theta \in[0,2 \pi]^{n}} \max _{1 \leq j \leq n} \frac{\left(-\left(u_{j}(t, R, \Theta)\right)_{t}^{\prime}\right)^{+}}{\frac{r_{j}}{r^{*}} l_{j}^{2}\left(\frac{t}{r^{*}} R e^{i \Theta}\right)} \leq C,
$$

then

$$
\varlimsup_{|R| \rightarrow 1-0} \frac{\ln \max \left\{\mid F(z): \quad z \in \mathbb{T}^{n}(\mathbf{0}, R)\right\}}{\max _{\Theta \in[0,2 \pi]^{n}} \int_{0}^{1}\left\langle R, \mathbf{L}\left(\tau R e^{i \Theta}\right)\right\rangle d \tau} \leq(C+1) N+1 .
$$

Proof. By Lemma 3 if $\mathbf{L}$ satisfies (1) then

$$
\max _{\Theta \in[0,2 \pi]^{n}} \int_{0}^{1}\left\langle R, \mathbf{L}\left(\tau R e^{i \Theta}\right)\right\rangle d \tau \rightarrow+\infty \text { as }|R| \rightarrow 1-0 .
$$

Denote $\widetilde{\beta}(t)=\sum_{j=1}^{n} \alpha_{j} l_{j}\left(A t e^{i \Theta}\right)$. If, in addition, (28) holds then for some $S^{*},\left\|S^{*}\right\| \leq N$ and $\widetilde{S},\|\widetilde{S}\| \leq N$,

$$
\begin{gathered}
\frac{\gamma(t)}{\widetilde{\beta}(t)}=\frac{\sum_{j=1}^{n} \frac{s_{j}^{*}\left(-u_{j}^{\prime}(t)\right)^{+}}{l_{j}\left(A t e^{i \Theta}\right)}}{\sum_{j=1}^{n} \alpha_{j} l_{j}\left(A t e^{i \Theta}\right)} \leq \sum_{j=1}^{n} s_{j}^{*} \frac{\left(-u_{j}^{\prime}(t)\right)^{+}}{\alpha_{j} l_{j}^{2}\left(A t e^{i \Theta}\right)} \leq \sum_{j=1}^{n} s_{j}^{*} \cdot C \leq N C, \\
\frac{\beta(t)}{\widetilde{\beta}(t)}=\frac{\sum_{j=1}^{n} \alpha_{j}\left(\tilde{s}_{j}+1\right) l_{j}\left(A t e^{i \Theta}\right)}{\sum_{j=1}^{n} \alpha_{j} l_{j}\left(A t e^{i \Theta}\right)}=1+\frac{\sum_{j=1}^{n} \alpha_{j} \tilde{s}_{j} l_{j}\left(A t e^{i \Theta}\right)}{\sum_{j=1}^{n} \alpha_{j} l_{j}\left(A t e^{i \Theta}\right)} \leq 1+\sum_{j=1}^{n} \tilde{s}_{j} \leq 1+N .
\end{gathered}
$$

But $\left|F\left(A t e^{i \Theta}\right)\right| \leq g(t) \leq g(0) \exp \int_{0}^{t}(\beta(\tau)+\gamma(\tau)) d \tau$ and $r^{*} A=R$. Putting $t=r^{*}$ and taking into account $(30)$, we obtain

$$
\ln \max \left\{\mid F(z): \quad z \in \mathbb{T}^{n}(\mathbf{0}, R)\right\}=\ln \max _{\Theta \in[0,2 \pi]^{n}}\left|F\left(R e^{i \Theta}\right)\right| \leq \ln \max _{\Theta \in[0,2 \pi]^{n}} g\left(r^{*}\right) \leq
$$




$$
\begin{gathered}
\leq \ln g(0)+\max _{\Theta \in[0,2 \pi]^{n}} \int_{0}^{r^{*}}(\beta(\tau)+\gamma(\tau)) d \tau \leq \ln g(0)+(N C+N+1) \max _{\Theta \in[0,2 \pi]^{n}} \int_{0}^{r^{*}} \widetilde{\beta}(\tau) d \tau= \\
=\ln g(0)+(N C+N+1) \max _{\Theta \in[0,2 \pi]^{n}} \int_{0}^{r^{*}} \sum_{j=1}^{n} \alpha_{j} l_{j}\left(A \tau e^{i \Theta}\right) d \tau= \\
=\ln g(0)+(N C+N+1) \max _{\Theta \in[0,2 \pi]^{n}} \int_{0}^{r^{*}} \sum_{j=1}^{n} \frac{r_{j}}{r^{*}} l_{j}\left(\frac{\tau}{r^{*}} R e^{i \Theta}\right) d \tau= \\
=\ln g(0)+(N C+N+1) \max _{\Theta \in[0,2 \pi]^{n}} \int_{0}^{1} \sum_{j=1}^{n} r_{j} l_{j}\left(\tau R e^{i \Theta}\right) d \tau .
\end{gathered}
$$

Thus, we conclude that (29) holds.

Theorem 9. Let $\mathbf{L}\left(R e^{i \Theta}\right)$ be a positive continuously differentiable function in each variable $r_{k}, k \in\{1, \ldots, n\},|R|<1, \Theta \in[0,2 \pi]^{n}$. If the function $\mathbf{L}$ satisfies (1) and an analytic function $F$ in $\mathbb{B}^{n}$ has bounded $\mathbf{L}$-index $N=N(F, \mathbf{L})$ in joint variables and

$$
r^{*}\left(-\left(u_{j}(t, R, \Theta)\right)_{t=r^{*}}^{\prime}\right)^{+} /\left(r_{j} l_{j}^{2}\left(R e^{i \Theta}\right)\right) \rightarrow 0
$$

uniformly in all $\Theta \in[0,2 \pi]^{n}, j \in\{1, \ldots, n\}$, as $|R| \rightarrow 1-0$ then

$$
\varlimsup_{|R| \rightarrow 1-0} \frac{\ln \max \left\{\mid F(z): \quad z \in \mathbb{T}^{n}(\mathbf{0}, R)\right\}}{\max _{\Theta \in[0,2 \pi]^{n}} \int_{0}^{1}\left\langle R, \mathbf{L}\left(\tau R e^{i \Theta}\right)\right\rangle d \tau} \leq N+1 .
$$

Estimate (32) can be deduced by analogy to the proof of Theorem 8 .

If $\mathbf{L}(z)=\mathbf{L}(R)$ then $(31)$ can be written in a simplified form.

Corollary 3. Let $\mathbf{L}(R)$ be a positive continuously differentiable function in each variable $r_{k}, k \in\{1, \ldots, n\},|R|<1$. If the function $\mathbf{L}$ satisfies $(1)$ and an analytic function $F$ in $\mathbb{B}^{n}$ has bounded $\mathbf{L}$-index $N=N(F, \mathbf{L})$ in joint variables and for every $j \in\{1, \ldots, n\}$

$$
\frac{\left\langle R, \nabla l_{j}(R)\right\rangle}{r_{j} l_{j}^{2}(R)} \rightarrow 0, \text { as }|R| \rightarrow 1-0
$$

then

$$
\varlimsup_{|R| \rightarrow 1-0} \frac{\ln \max \left\{|F(z)|: \quad z \in T^{n}(\mathbf{0}, R)\right\}}{\int_{0}^{1}\langle R, \mathbf{L}(\tau R)\rangle d \tau} \leq N+1,
$$

where $\nabla l_{j}(R)=\left(\frac{\partial l_{j}(R)}{\partial r_{1}}, \ldots, \frac{\partial l_{j}(R)}{r_{n}}\right)$.

Our main result in this section is the following

Theorem 10. Let $\mathbf{L}(R)=\left(l_{1}(R), \ldots, l_{n}(R)\right), l_{j}(R)$ be a positive continuously differentiable non-decreasing function in each variable $r_{k}, k \in\{1, \ldots, n\},|R|<1$. If the function $\mathbf{L}$ satisfies (1) and an analytic function $F$ in $\mathbb{B}^{n}$ has bounded $\mathbf{L}$-index $N=N(F, \mathbf{L})$ in joint variables then

$$
\varlimsup_{|R| \rightarrow 1-0} \frac{\ln \max \left\{|F(z)|: \quad z \in T^{n}(\mathbf{0}, R)\right\}}{\int_{0}^{1}\langle R, \mathbf{L}(\tau R)\rangle d \tau} \leq N+1 .
$$


This statement is a consequence of Theorem 9, which is obtained for a more general function $\mathbf{L}$.

We will write $u(r, \theta)=l\left(r e^{i \theta}\right)$. Theorem 7 implies the following proposition for $n=1$.

Corollary 4. Let $l\left(r e^{i \theta}\right)$ be a positive continuously differentiable function in variable $r \in$ $[0,1)$ for every $\theta \in[0,2 \pi]$. If an analytic function $f$ in $\mathbb{D}$ has bounded l-index $N=N(f, l)$ and $\varlimsup_{r \rightarrow 1-0} \max _{\theta \in[0,2 \pi]} \frac{\left(-u_{r}^{\prime}(r, \theta)\right)^{+}}{l^{2}\left(r e^{i \theta}\right)}=C \geq 0$ then

$$
\varlimsup_{r \rightarrow 1-0} \frac{\ln \max \{|f(z):| z \mid=r\}}{\max _{\theta \in[0,2 \pi]} \int_{0}^{r} l\left(\tau e^{i \theta}\right) d \tau} \leq(C+1) N+1 .
$$

Estimate (32) is sharp. It is easy to check for these functions $F(z)=\exp \left\{\frac{1}{\left(1-z_{1}\right)\left(1-z_{2}\right)}\right\}$, $l_{1}\left(z_{1}, z_{2}\right)=\frac{1}{\left(1-\left|z_{1}\right|\right)^{2}(1-|z|)}, l_{2}\left(z_{1}, z_{2}\right)=\frac{1}{(1-|z|)\left(1-\left|z_{2}\right|\right)^{2}}$. Hence, we have $N\left(F, \mathbf{L}, \mathbb{B}^{n}\right)=0$ and $\ln \max \left\{|F(z)|: z \in T^{2}(\mathbf{0}, R)\right\}=\frac{1}{\left(1-r_{1}\right)\left(1-r_{2}\right)}$.

6. Bounded L-index in joint variables in a bounded domain. By $\bar{G}$ we denote the closure of a domain $G$. The following result is generalization of one-dimensional propositions from $[26,35]$.

Theorem 11. Let $F(z)$ be an analytic function in $\mathbb{B}^{n}, G$ be a bounded domain in $\mathbb{B}^{n}$, $d=\inf _{z \in \bar{G}}(1-|z|)>0$ and $\beta>\sqrt{n}$. If for every $j \in\{1, \ldots, n\} l_{j}: \mathbb{B}^{n} \rightarrow \mathbb{R}_{+}$is a continuous function satisfying $l_{j}(z) \geq \frac{\beta}{d}$ for all $z \in \mathbb{B}^{n}$ then there exists $m \in \mathbb{Z}_{+}$such that for all $z \in \bar{G}$ and $J=\left(j_{1}, j_{2}, \ldots, j_{n}\right) \in \mathbb{Z}_{+}^{n}$

$$
\frac{\left|F^{(J)}(z)\right|}{J ! \mathbf{L}^{J}(z)} \leq \max \left\{\frac{\left|F^{(K)}(z)\right|}{K ! \mathbf{L}^{K}(z)}: K \in \mathbb{Z}_{+}^{n},\|K\| \leq m\right\}
$$

where $\mathbf{L}(z)=\left(l_{1}(z), \ldots, l_{n}(z)\right)$.

Proof. If $F(z) \equiv 0$ then (34) is obvious. Let $F(z) \not \equiv 0$. For every fixed $z^{0} \in \bar{G} \frac{\left|F^{(J)}\left(z^{0}\right)\right|}{J ! \mathbf{L}^{J}\left(z^{0}\right)}$ is the modulus of a coefficient of the power series expansion of the function $F(z), z \in \mathbb{T}^{n}\left(z^{0}, \frac{R_{0}}{\mathbf{L}\left(z^{0}\right)}\right)$, where $\left|R_{0}\right|=\sqrt{n}$. Since $F(z)$ is analytic, for every $z^{0} \in \bar{G} \frac{\left|F^{(J)}\left(z^{0}\right)\right|}{J ! \mathbf{L}^{J}\left(z^{0}\right)} \rightarrow 0$ as $\|J\| \rightarrow \infty$, i. e. there exists $m_{0}=m\left(z^{0}\right)$, for which inequality (34) holds.

Assume on the contrary, that the set of $m_{0}$ is not uniformly bounded in $z^{0}: \sup _{z^{0} \in \bar{G}} m_{0}=$ $+\infty$. Hence, for every $m \in \mathbb{Z}_{+}$there exist $z_{m} \in \bar{G}$ and $J^{m} \in \mathbb{Z}_{+}^{n}$

$$
\frac{\left|F^{\left(J^{m}\right)}\left(z^{m}\right)\right|}{J^{m} ! \mathbf{L}^{J^{m}}\left(z_{m}\right)}>\max \left\{\frac{\left|F^{(K)}\left(z^{m}\right)\right|}{K ! \mathbf{L}^{K}\left(z^{m}\right)}: K \in \mathbb{Z}_{+}^{n},\|K\| \leq m\right\} .
$$

Since $z^{m} \in \bar{G}$, there exists a subsequence $z^{\prime m} \rightarrow z^{\prime} \in \bar{G}$ as $m \rightarrow+\infty$. By Cauchy's integral formula for any $J \in \mathbb{Z}_{+}^{n}$

$$
\frac{F^{(J)}\left(z^{0}\right)}{J !}=\frac{1}{(2 \pi i)^{n}} \int_{z \in \mathbb{T}^{n}\left(z^{0}, R\right)} \frac{F(z)}{\left(z-z^{0}\right)^{J+1}} d z .
$$

We rewrite (35) in the form

$$
\max \left\{\frac{\left|F^{(K)}\left(z^{m}\right)\right|}{K ! \mathbf{L}^{K}\left(z^{m}\right)}: \quad K \in \mathbb{Z}_{+}^{n},\|K\| \leq m\right\} \leq
$$




$$
\leq \frac{1}{(2 \pi)^{n} \mathbf{L}^{J^{m}}\left(z^{m}\right)} \int_{z \in \mathbb{T}^{n}\left(z^{0}, \frac{R}{\mathbf{L}\left(z^{m}\right)}\right)} \frac{|F(z)|}{\left|z-z^{m}\right|^{J^{m}+\mathbf{1}}}|d z| \leq \frac{1}{R^{J^{m}}} \max \left\{|F(z)|: z \in G_{R}\right\},
$$

where $G_{R}=\bigcup_{z^{*} \in \bar{G}} D^{n}\left[z^{*}, \frac{R}{\mathbf{L}\left(z^{*}\right)}\right],|R| \leq \beta$. We choose $R$ such that $r_{j}>1$ i. e. $|R|>\sqrt{n}$. Taking the limit in (36) as $m \rightarrow \infty$ we deduce

$$
\forall K \in \mathbb{Z}_{+}^{n} \frac{\left|F^{(K)}\left(z^{\prime}\right)\right|}{K ! \mathbf{L}^{K}\left(z^{\prime}\right)} \leq \lim _{m \rightarrow \infty} \frac{1}{R^{J^{m}}} \max \left\{|F(z)|: z \in G_{R}\right\}=0 .
$$

as $m \rightarrow+\infty$. Thus, all partial derivatives of the function $F$ at point $z^{\prime}$ equals 0 . By uniqueness theorem $F(z) \equiv 0$. It is impossible.

Remark 1. A similar proposition for analytic functions in $\mathbb{B}^{n}$ of bounded $L$-index in a direction $\mathbf{b} \in \mathbb{C}^{n} \backslash\{\mathbf{0}\}$ is valid under the additional assumption $\forall z \in \bar{G} F(z+t \mathbf{b}) \not \equiv 0$, where $t \in \mathbb{C}($ see $[4,5])$.

7. Exhaustion of unit ball by balls of lesser radii. Denote $\ell(z)=\min _{1 \leq j \leq n} l_{j}(z)$, $\mathcal{L}(z)=\max _{1 \leq j \leq n} l_{j}(z)$. Obviously, that $\ell(z) \leq \mathcal{L}(z)$.

By $Q^{\prime}\left(\mathbb{B}^{n}\right)$ we denote the class of functions $\mathbf{L}$, which satisfy the condition

$$
(\forall r \in[0, \beta], j \in\{1, \ldots, n\}): 0<\lambda_{1, j}(r) \leq \lambda_{2, j}(r)<\infty
$$

where

$$
\begin{gathered}
\lambda_{1, j}(r)=\inf _{z^{0} \in \mathbb{B}^{n}} \inf \left\{\frac{l_{j}(z)}{l_{j}\left(z^{0}\right)}: z \in \mathbb{B}^{n}\left[z^{0}, r / \ell\left(z^{0}\right)\right]\right\} \\
\lambda_{2, j}(r)=\sup _{z^{0} \in \mathbb{B}^{n}} \sup \left\{\frac{l_{j}(z)}{l_{j}\left(z^{0}\right)}: z \in \mathbb{B}^{n}\left[z^{0}, r / \ell\left(z^{0}\right)\right]\right\} . \\
\Lambda_{1}(r)=\left(\lambda_{1,1}(r), \ldots, \lambda_{1, n}(r)\right), \Lambda_{2}(r)=\left(\lambda_{2,1}(r), \ldots, \lambda_{2, n}(r)\right) .
\end{gathered}
$$

These denotations of $\lambda_{1, j}(r), \lambda_{2, j}(r), \Lambda_{1}(r), \Lambda_{2}(r)$ are valid in this section only. In other sections their meanings are defined in (4)-(5).

The following theorem is basic in the theory of functions of bounded index. It was necessary to prove more efficient criteria of index boundedness which describe a behavior of maximum modulus on a disc or a behavior of logarithmic derivative (see $[3,5,25,29,32,35])$. All cited papers used a polydisc exhaustion in $\mathbb{C}^{n}$ or a disc exhaustion in $\mathbb{C}$.

Theorem 12. Let $\mathbf{L} \in Q^{\prime}\left(\mathbb{B}^{n}\right)$. In order that an analytic function $F$ in $\mathbb{B}^{n}$ be of bounded $\mathbf{L}$-index in joint variables it is necessary that for each $r \in(0, \beta]$ there exist $n_{0} \in \mathbb{Z}_{+}, p_{0}>0$ such that for every $z^{0} \in \mathbb{B}^{n}$ there exists $K^{0} \in \mathbb{Z}_{+}^{n},\left\|K^{0}\right\| \leq n_{0}$, satisfying

$$
\max \left\{\frac{\left|F^{(K)}(z)\right|}{K ! \mathbf{L}^{K}(z)}:\|K\| \leq n_{0}, z \in \mathbb{B}^{n}\left[z^{0}, r / \mathcal{L}\left(z^{0}\right)\right]\right\} \leq p_{0} \frac{\left|F^{\left(K^{0}\right)}\left(z^{0}\right)\right|}{K^{0} ! \mathbf{L}^{K^{0}}\left(z^{0}\right)}
$$

and it is sufficient that for each $r \in(0, \beta]$ there exist $n_{0} \in \mathbb{Z}_{+}, p_{0}>0$ such that for every $z^{0} \in \mathbb{B}^{n}$ there exists $K^{0} \in \mathbb{Z}_{+}^{n},\left\|K^{0}\right\| \leq n_{0}$, satisfying

$$
\max \left\{\frac{\left|F^{(K)}(z)\right|}{K ! \mathbf{L}^{K}(z)}:\|K\| \leq n_{0}, z \in \mathbb{B}^{n}\left[z^{0}, r / \ell\left(z^{0}\right) \mid\right]\right\} \leq p_{0} \frac{\left|F^{\left(K^{0}\right)}\left(z^{0}\right)\right|}{K^{0} ! \mathbf{L}^{K^{0}}\left(z^{0}\right)} .
$$


Proof. Let $F$ be of bounded L-index in joint variables with $N=N\left(F, \mathbf{L}, \mathbb{B}^{n}\right)<\infty$. For every $r \in(0, \beta]$ we put

$$
q=q(r)=\left[2(N+1) r \sqrt{n} \prod_{j=1}^{n}\left(\lambda_{1, j}(r)\right)^{-N}\left(\lambda_{2, j}(r)\right)^{N+1}\right]+1,
$$

where $[x]$ is integer part of the real number $x$, i.e. the floor function. For $p \in\{0, \ldots, q\}$ and $z^{0} \in \mathbb{B}^{n}$ we denote

$$
\begin{aligned}
& S_{p}\left(z^{0}, r\right)=\max \left\{\frac{\left|F^{(K)}(z)\right|}{K ! \mathbf{L}^{K}(z)}:\|K\| \leq N, z \in \mathbb{B}^{n}\left[z^{0}, \frac{p r}{q \mathcal{L}\left(z^{0}\right)}\right]\right\}, \\
& S_{p}^{*}\left(z^{0}, r\right)=\max \left\{\frac{\left|F^{(K)}(z)\right|}{K ! \mathbf{L}^{K}\left(z^{0}\right)}:\|K\| \leq N, z \in \mathbb{B}^{n}\left[z^{0}, \frac{p r}{q \mathcal{L}\left(z^{0}\right)}\right]\right\} .
\end{aligned}
$$

Using (4) and $\mathbb{B}^{n}\left[z^{0}, \frac{p r}{q \ell\left(z^{0}\right)}\right] \subset \mathbb{B}^{n}\left[z^{0}, \frac{r}{\mathcal{L}\left(z^{0}\right)}\right]$, we have

$$
\begin{gathered}
S_{p}\left(z^{0}, r\right)=\max \left\{\frac{\left|F^{(K)}(z)\right|}{K ! \mathbf{L}^{K}(z)} \frac{\mathbf{L}^{K}\left(z^{0}\right)}{\mathbf{L}^{K}\left(z^{0}\right)}:\|K\| \leq N, z \in \mathbb{B}^{n}\left[z^{0}, \frac{p r}{q \mathcal{L}\left(z^{0}\right)}\right]\right\} \leq \\
\leq S_{p}^{*}\left(z^{0}, r\right) \max \left\{\prod_{j=1}^{n} \frac{l_{j}^{N}\left(z^{0}\right)}{l_{j}^{N}(z)}: z \in \mathbb{B}^{n}\left[z^{0}, \frac{p r}{q \mathcal{L}\left(z^{0}\right)}\right]\right\} \leq S_{p}^{*}\left(z^{0}, r\right) \prod_{j=1}^{n}\left(\lambda_{1, j}(r)\right)^{-N} .
\end{gathered}
$$

and, using (5), we obtain

$$
\begin{gathered}
S_{p}^{*}\left(z^{0}, r\right)=\max \left\{\frac{\left|F^{(K)}(z)\right|}{K ! \mathbf{L}^{K}(z)} \frac{\mathbf{L}^{K}(z)}{\mathbf{L}^{K}\left(z^{0}\right)}:\|K\| \leq N, z \in \mathbb{B}^{n}\left[z^{0}, \frac{p r}{q \mathcal{L}\left(z^{0}\right)}\right]\right\} \leq \\
\leq \max \left\{\frac{\left|F^{(K)}(z)\right|}{K ! \mathbf{L}^{K}(z)}\left(\Lambda_{2}(r)\right)^{K}:\|K\| \leq N, z \in \mathbb{B}^{n}\left[z^{0}, \frac{p r}{q \mathcal{L}\left(z^{0}\right)}\right]\right\} \leq S_{p}\left(z^{0}, r\right) \prod_{j=1}^{n}\left(\lambda_{2, j}(r)\right)^{N} .
\end{gathered}
$$

Let $K^{(p)}$ with $\left\|K^{(p)}\right\| \leq N$ and $z^{(p)} \in \mathbb{B}^{n}\left[z^{0}, \frac{p r}{q \mathcal{L}\left(z^{0}\right)}\right]$ be such that

$$
S_{p}^{*}\left(z^{0}, r\right)=\frac{\left|F^{\left(K^{(p)}\right)}\left(z^{(p)}\right)\right|}{K^{(p) ! \mathbf{L}^{K(p)}}\left(z^{0}\right)} .
$$

Since by the maximum principle $z^{(p)} \in \mathbb{S}_{n}\left(z^{0}, \frac{p r}{q \mathcal{L}\left(z^{0}\right)}\right)$, we have $z^{(p)} \neq z^{0}$. We choose

$$
\widetilde{z}_{j}^{(p)}=z_{j}^{0}+\frac{p-1}{p}\left(z_{j}^{(p)}-z_{j}^{0}\right), j \in\{1, \ldots, n\} .
$$

Then we have

$$
\begin{gathered}
\left|\widetilde{z}^{(p)}-z^{0}\right|=\frac{p-1}{p}\left|z^{(p)}-z^{0}\right|=\frac{p-1}{p} \frac{p r}{q \mathcal{L}\left(z^{0}\right)} \\
\left|\widetilde{z}^{(p)}-z^{(p)}\right|=\left|z^{0}+\frac{p-1}{p}\left(z^{(p)}-z^{0}\right)-z^{(p)}\right|=\frac{1}{p}\left|z^{0}-z^{(p)}\right|=\frac{1}{p} \frac{p r}{q \ell\left(z^{0}\right)}=\frac{r}{q \mathcal{L}\left(z^{0}\right)} .
\end{gathered}
$$

From (45) we obtain $\widetilde{z}^{(p)} \in \mathbb{B}^{n}\left[z^{0}, \frac{(p-1) r}{q \mathcal{L}\left(z^{0}\right)}\right]$ and

$$
S_{p-1}^{*}\left(z^{0}, r\right) \geq \frac{\left|F^{\left(K^{(p)}\right)}\left(\widetilde{z}^{(p)}\right)\right|}{K^{(p) ! \mathbf{L}^{K^{(p)}}\left(z^{0}\right)}} .
$$


From (44) it follows that

$$
\begin{aligned}
& 0 \leq S_{p}^{*}\left(z^{0}, r\right)-S_{p-1}^{*}\left(z^{0}, r\right) \leq \frac{\left|F^{\left(K^{(p)}\right)}\left(z^{(p)}\right)\right|-\left|F^{\left(K^{(p)}\right)}\left(\widetilde{z}^{(p)}\right)\right|}{K^{(p)} ! \mathbf{L}^{K^{(p)}}\left(z^{0}\right)}= \\
& =\frac{1}{K^{(p) ! \mathbf{L}^{K(p)}\left(z^{0}\right)}} \int_{0}^{1} \frac{d}{d t}\left|F^{\left(K^{(p)}\right)}\left(\widetilde{z}^{(p)}+t\left(z^{(p)}-\widetilde{z}^{(p)}\right)\right)\right| d t \leq \\
& \leq \frac{1}{K^{(p) ! \mathbf{L}^{K^{(p)}}}\left(z^{0}\right)} \int_{0}^{1} \sum_{j=1}^{n}\left|z_{j}^{(p)}-\widetilde{z}_{j}^{(p)}\right|\left|F^{\left(K^{(p)}+\mathbf{1}_{j}\right)}\left(\widetilde{z}^{(p)}+t\left(z^{(p)}-\widetilde{z}^{(p)}\right)\right)\right| d t= \\
& =\frac{1}{K^{(p) ! \mathbf{L}^{K^{(p)}}\left(z^{0}\right)}} \sum_{j=1}^{n}\left|z_{j}^{(p)}-\widetilde{z}_{j}^{(p)}\right|\left|F^{\left(K^{(p)}+\mathbf{1}_{j}\right)}\left(\widetilde{z}^{(p)}+t^{*}\left(z^{(p)}-\widetilde{z}^{(p)}\right)\right)\right|,
\end{aligned}
$$

where $0 \leq t^{*} \leq 1, \widetilde{z}^{(p)}+t^{*}\left(z^{(p)}-\widetilde{z}^{(p)}\right) \in \mathbb{B}^{n}\left(z^{0}, \frac{p r}{q \mathcal{L}\left(z^{0}\right)}\right)$. For $z \in \mathbb{B}^{n}\left(z^{0}, \frac{p r}{q \mathcal{L}\left(z^{0}\right)}\right)$ and $J \in \mathbb{Z}_{+}^{n}$, $\|J\| \leq N+1$ we have

$$
\begin{gathered}
\quad \frac{\left|F^{(J)}(z)\right| \mathbf{L}^{J}(z)}{J ! \mathbf{L}^{J}\left(z^{0}\right) \mathbf{L}^{J}(z)} \leq\left(\Lambda_{2}(r)\right)^{J} \max \left\{\frac{\left|F^{(K)}(z)\right|}{K ! \mathbf{L}^{K}(z)}:\|K\| \leq N\right\} \leq \\
\leq \prod_{j=1}^{n}\left(\lambda_{2, j}(r)\right)^{N+1}\left(\lambda_{1, j}(r)\right)^{-N} \max \left\{\frac{\left|F^{(K)}(z)\right|}{K ! \mathbf{L}^{K}\left(z^{0}\right)}:\|K\| \leq N\right\} \leq \\
\leq \prod_{j=1}^{n}\left(\lambda_{2, j}(r)\right)^{N+1}\left(\lambda_{1, j}(r)\right)^{-N} S_{p}^{*}\left(z^{0}, r\right) .
\end{gathered}
$$

From (47) and (46) we obtain

$$
\begin{gathered}
0 \leq S_{p}^{*}\left(z^{0}, r\right)-S_{p-1}^{*}\left(z^{0}, r\right) \leq \\
\leq \prod_{j=1}^{n}\left(\lambda_{2, j}(r)\right)^{N+1}\left(\lambda_{1, j}(r)\right)^{-N} S_{p}^{*}\left(z^{0}, r\right) \sum_{j=1}^{n}\left(k_{j}^{(p)}+1\right) l_{j}\left(z^{0}\right)\left|z_{j}^{(p)}-\widetilde{z}_{j}^{(p)}\right|= \\
=\prod_{j=1}^{n}\left(\lambda_{2, j}(r)\right)^{N+1}\left(\lambda_{1, j}(r)\right)^{-N} S_{p}^{*}\left(z^{0}, r\right)(N+1) \sum_{j=1}^{n} l_{j}\left(z^{0}\right)\left|z_{j}^{(p)}-\widetilde{z}_{j}^{(p)}\right| \leq \\
\leq \prod_{j=1}^{n}\left(\lambda_{2, j}(r)\right)^{N+1}\left(\lambda_{1, j}(r)\right)^{-N}(N+1) S_{p}^{*}\left(z^{0}, R\right) \sqrt{n} \mathcal{L}\left(z^{0}\right)\left|z^{(p)}-\widetilde{z}^{(p)}\right|= \\
=\prod_{j=1}^{n}\left(\lambda_{2, j}(r)\right)^{N+1}\left(\lambda_{1, j}(r)\right)^{-N} \sqrt{n} \frac{(N+1) r}{q(r)} S_{p}^{*}\left(z^{0}, R\right) \leq \frac{1}{2} S_{p}^{*}\left(z^{0}, R\right) .
\end{gathered}
$$

This inequality implies $S_{p}^{*}\left(z^{0}, r\right) \leq 2 S_{p-1}^{*}\left(z^{0}, r\right)$, and in view of inequalities (43) and (44) we have

$$
S_{p}\left(z^{0}, r\right) \leq 2 \prod_{j=1}^{n}\left(\lambda_{1, j}(r)\right)^{-N} S_{p-1}^{*}\left(z^{0}, r\right) \leq 2 \prod_{j=1}^{n}\left(\lambda_{1, j}(r)\right)^{-N}\left(\lambda_{2, j}(r)\right)^{N} S_{p-1}\left(z^{0}, r\right)
$$

Therefore,

$$
\max \left\{\frac{\left|F^{(K)}(z)\right|}{K ! \mathbf{L}^{K}(z)}:\|K\| \leq N, z \in \mathbb{B}^{n}\left[z^{0}, \frac{p r}{q \mathcal{L}\left(z^{0}\right)}\right]\right\}=S_{q}\left(z^{0}, r\right) \leq
$$




$$
\begin{gathered}
\leq 2 \prod_{j=1}^{n}\left(\lambda_{1, j}(r)\right)^{-N}\left(\lambda_{2, j}(r)\right)^{N} S_{q-1}\left(z^{0}, r\right) \leq \ldots \leq\left(2 \prod_{j=1}^{n}\left(\lambda_{1, j}(r)\right)^{-N}\left(\lambda_{2, j}(r)\right)^{N}\right)^{q} S_{0}\left(z^{0}, r\right)= \\
=\left(2 \prod_{j=1}^{n}\left(\lambda_{1, j}(r)\right)^{-N}\left(\lambda_{2, j}(r)\right)^{N}\right)^{q} \max \left\{\frac{\left|F^{(K)}\left(z^{0}\right)\right|}{K ! \mathbf{L}^{K}\left(z^{0}\right)}:\|K\| \leq N\right\} .
\end{gathered}
$$

From (48) we obtain inequality (41) with $p_{0}=\left(2 \prod_{j=1}^{n}\left(\lambda_{1, j}(r)\right)^{-N}\left(\lambda_{2, j}(r)\right)^{N}\right)^{q}$ and some $K^{0}$ with $\left\|K^{0}\right\| \leq N$. The necessity of condition (41) is proved.

Now we prove the sufficiency. Suppose that for every $r \in(0, \beta]$ there exist $n_{0} \in \mathbb{Z}_{+}$, $p_{0}>1$ such that for all $z_{0} \in \mathbb{B}^{n}$ and some $K^{0} \in \mathbb{Z}_{+}^{n},\left\|K^{0}\right\| \leq n_{0}$, the inequality (42) holds.

We write Cauchy's formula for a ball (see [37, p. 109] or [31, p. 349]) as following $\forall z^{0} \in \mathbb{B}^{n}$ $\forall K \in \mathbb{Z}_{+}^{n} \forall S \in \mathbb{Z}_{+}^{n} \forall z \in \mathbb{B}^{n}\left(z^{0}, r / \ell\left(z^{0}\right)\right)$

$$
F^{(K+S)}(z)=\frac{(n+\|S\|-1) !}{(n-1) !} \int_{\mathbb{S}^{n}\left(z^{0}, r / \ell\left(z^{0}\right)\right)} \frac{\left|\xi-z^{0}\right|\left(\overline{\xi-z^{0}}\right)^{S} F^{(K)}(\xi)}{\left(\left|\xi-z^{0}\right|^{2}-\left\langle z-z^{0}, \xi-z^{0}\right\rangle\right)^{n+\|S\|}} d \sigma(\xi),
$$

where $d \sigma(\xi)$ is the normalized surface measure on $\mathbb{S}_{n}$, so that $\sigma\left(\mathbb{S}_{n}(\mathbf{0}, 1)\right)=1$. Put $z=z^{0}$ :

$$
F^{(K+S)}\left(z^{0}\right)=\frac{(n+\|S\|-1) !}{(n-1) !} \int_{\mathbb{S}^{n}\left(z^{0}, r / \ell\left(z^{0}\right)\right)} \frac{\left(\overline{\xi-z^{0}}\right)^{S} F^{(K)}(\xi)}{\left|\xi-z^{0}\right|^{2(n+\|S\|)-1}} d \sigma(\xi)
$$

Therefore, applying (42), we have

$$
\begin{aligned}
& \left|F^{(K+S)}\left(z^{0}\right)\right| \leq \frac{(n+\|S\|-1) !}{(n-1) !} \int_{\mathbb{S}^{n}\left(z^{0}, r / \ell\left(z^{0}\right)\right)} \frac{\left|\left(\xi-z^{0}\right)^{S}\right|\left|F^{(K)}(\xi)\right|}{\left|\xi-z^{0}\right|^{2(n+\|S\|)-1}} d \sigma(\xi) \leq \\
& \leq\left(\frac{\ell\left(z^{0}\right)}{r}\right)^{2(n+\|S\|)-1} \frac{(n+\|S\|-1) !}{(n-1) !} \int_{\mathbb{S}^{n}\left(z^{0}, r / \ell\left(z^{0}\right)\right)} \frac{\left|\left(\xi-z^{0}\right)^{S} \| F^{(K)}(\xi)\right| K ! \mathbf{L}^{K}(\xi)}{K ! \mathbf{L}^{K}(\xi)} d \sigma(\xi) \leq \\
& \leq p_{0}\left(\frac{\ell\left(z^{0}\right)}{r}\right)^{2(n+\|S\|)-1} \frac{(n+\|S\|-1) !}{(n-1) !} \int_{\mathbb{S}^{n}\left(z^{0}, r / \ell\left(z^{0}\right)\right)} \frac{\left|\left(\xi-z^{0}\right)^{S} \| F^{\left(K^{0}\right)}\left(z^{0}\right)\right| K ! \mathbf{L}^{K}(z)}{K^{0} ! \mathbf{L}^{K^{0}}\left(z^{0}\right)} d \sigma(\xi) \leq \\
& \leq p_{0}\left(\frac{\ell\left(z^{0}\right)}{r}\right)^{2(n+\|S\|)-1} \frac{(n+\|S\|-1) !}{(n-1) !} \frac{\left|F^{\left(K^{0}\right)}\left(z^{0}\right)\right| K ! \prod_{j=1}^{n} \lambda_{2, j}^{n_{0}}(r) \mathbf{L}^{K}\left(z^{0}\right)}{K^{0} ! \mathbf{L}^{K^{0}}\left(z^{0}\right)} \times \\
& \times \int_{\mathbb{S}^{n}\left(z^{0}, r / \ell\left(z^{0}\right)\right)}\left|\left(\xi-z^{0}\right)^{S}\right| d \sigma(\xi) \leq \\
& \leq p_{0}\left(\frac{\ell\left(z^{0}\right)}{r}\right)^{\|S\|} \frac{(n+\|S\|-1) !}{(n-1) !} \frac{\left|F^{\left(K^{0}\right)}\left(z^{0}\right)\right| K ! \prod_{j=1}^{n} \lambda_{2, j}^{n_{0}}(r) \mathbf{L}^{K}\left(z^{0}\right)}{K^{0} ! \mathbf{L}^{K^{0}}\left(z^{0}\right)} \times \\
& \times \int_{\mathbb{S}^{n}\left(z^{0}, r / \ell\left(z^{0}\right)\right)} \frac{\left|\left(\xi-z^{0}\right)^{S}\right|}{\left(r / \ell\left(z^{0}\right)\right)^{\|S\|}} d \sigma\left(\frac{\xi-z^{0}}{r / \ell\left(z^{0}\right)}\right) \leq \\
& \leq p_{0}\left(\frac{\ell\left(z^{0}\right)}{r}\right)^{\|S\|} \frac{(n+\|S\|-1) !}{(n-1) !} \frac{\left|F^{\left(K^{0}\right)}\left(z^{0}\right)\right| K ! \prod_{j=1}^{n} \lambda_{2, j}^{n_{0}}(r) \mathbf{L}^{K}\left(z^{0}\right)}{K^{0} ! \mathbf{L}^{K^{0}}\left(z^{0}\right)} \times
\end{aligned}
$$




$$
\begin{gathered}
\times \int_{\mathbb{S}^{n}(\mathbf{0}, 1)}\left|\xi^{S}\right| d \sigma(\xi)=p_{0}\left(\frac{\ell\left(z^{0}\right)}{r}\right)^{\|S\|} \frac{(n+\|S\|-1) !}{(n-1) !} \times \\
\times \frac{\left|F^{\left(K^{0}\right)}\left(z^{0}\right)\right| K ! \prod_{j=1}^{n} \lambda_{2, j}^{n_{0}}(r) \mathbf{L}^{K}\left(z^{0}\right)}{K^{0} ! \mathbf{L}^{K^{0}}\left(z^{0}\right)} \frac{\Gamma(n) \prod_{j=1}^{n} \Gamma\left(s_{j} / 2+1\right)}{\Gamma(n+\|S\| / 2)} .
\end{gathered}
$$

This implies

$$
\begin{gathered}
\frac{\left|F^{(K+S)}\left(z^{0}\right)\right|}{(K+S) ! \mathbf{L}^{K+S}\left(z^{0}\right)} \leq \\
\leq \frac{\left|F^{\left(K^{0}\right)}\left(z^{0}\right)\right|}{K^{0} ! \mathbf{L}^{K^{0}}\left(z^{0}\right)} p_{0}\left(\frac{\ell\left(z^{0}\right)}{r}\right)^{\|S\|} \frac{K ! \prod_{j=1}^{n} \lambda_{2, j}^{n_{0}}(r)(n+\|S\|-1) ! \prod_{j=1}^{n} \Gamma\left(s_{j} / 2+1\right)}{(K+S) ! \Gamma(n+\|S\| / 2) \mathbf{L}^{S}\left(z^{0}\right)} \leq \\
\leq \frac{\left|F^{\left(K^{0}\right)}\left(z^{0}\right)\right|}{K^{0} ! \mathbf{L}^{K^{0}}\left(z^{0}\right)} p_{0} \frac{K ! \prod_{j=1}^{n} \lambda_{2, j}^{n_{0}}(r)(n+\|S\|-1) ! \prod_{j=1}^{n} \Gamma\left(s_{j} / 2+1\right)}{(K+S) ! \Gamma(n+\|S\| / 2) r\|S\|}
\end{gathered}
$$

We choose $r>1$. Since $\|K\| \leq n_{0}$ the quantity $p_{0} K ! \prod_{j=1}^{n} \lambda_{2, j}^{n_{0}}(R)$ does not depend of $S$. Then there exists $n_{1}$ such that

$$
\frac{p_{0} K ! \prod_{j=1}^{n} \lambda_{2, j}^{n_{0}}(r)}{r^{\|S\|}} \leq 1 \text { for all }\|S\| \geq n_{1} .
$$

The asymptotic behavior of $\frac{(n+\|S\|-1) ! \prod_{j=1}^{n} \Gamma\left(s_{j} / 2+1\right)}{(K+S) ! \Gamma(n+\|S\| / \|) r\|S\|}$ is more difficult as $\|S\| \rightarrow+\infty$. Using the Stirling formula $\Gamma(m+1)=\sqrt{2 \pi m}\left(\frac{m}{e}\right)^{m}\left(1+\frac{\theta}{12 m}\right)$, where $\theta=\theta(m) \in[0,1]$, we obtain

$$
\begin{gathered}
\frac{(n+\|S\|-1) ! \prod_{j=1}^{n} \Gamma\left(s_{j} / 2+1\right)}{(K+S) ! \Gamma(n+\|S\| / 2) r\|S\|} \leq \frac{(n+\|S\|-1) ! \prod_{j=1}^{n} \Gamma\left(s_{j} / 2+1\right)}{S ! \Gamma(n+\|S\| / 2) r\|S\|} \\
=\frac{\sqrt{2 \pi(n+\|S\|-1)}\left(\frac{n+\|S\|-1}{e}\right)^{n+\|S\|-1} \prod_{j=1}^{n} \sqrt{2 \pi s_{j} / 2}\left(\frac{s_{j}}{2 e}\right)^{s_{j} / 2}}{\prod_{j=1}^{n} \sqrt{2 \pi s_{j}}\left(\frac{s_{j}}{e}\right)^{s_{j}} \sqrt{2 \pi(n+\|S\| / 2-1)}\left(\frac{n+\|S\| / 2-1}{e}\right)^{n+\|S\| / 2-1} r\|S\|} \times \\
\times \frac{\left(1+\frac{\theta(n+\|S\|-1)}{12(n+\|S\|-1)}\right) \prod_{j=1}^{n}\left(1+\frac{\theta\left(s_{j} / 2\right)}{12 s_{j} / 2}\right)}{\left(1+\frac{\theta(n+\|S\| / 2)}{12(n+\|S\| / 2)}\right) \prod_{j=1}^{n}\left(1+\frac{\theta\left(s_{j}\right)}{12 s_{j}}\right)} .
\end{gathered}
$$

Denoting

$$
\Theta(S)=\frac{\left(1+\frac{\theta(n+\|S\|-1)}{12(n+\|S\|-1)}\right) \prod_{j=1}^{n}\left(1+\frac{\theta\left(s_{j} / 2\right)}{12 s_{j} / 2}\right)}{\left(1+\frac{\theta(n+\|S\| / 2)}{12(n+\|S\| / 2)}\right) \prod_{j=1}^{n}\left(1+\frac{\theta\left(s_{j}\right)}{12 s_{j}}\right)}
$$

and simplifying the previous inequality we deduce

$$
\begin{gathered}
\frac{(n+\|S\|-1) ! \prod_{j=1}^{n} \Gamma\left(s_{j} / 2+1\right)}{(K+S) ! \Gamma(n+\|S\| / 2) r\|S\|} \leq \\
\leq \Theta(S) \frac{2^{(1-n) / 2} e^{-\|S\| / 2}}{r^{\|S\|}}\left(\frac{n-1+\|S\|}{n-1+\|S\| / 2}\right)^{n-1+\|S\| / 2} \cdot(n-1+\|S\|)^{\|S\| / 2} \times \\
\times \prod_{j=1}^{n}\left(\frac{e}{2 s_{j}}\right)^{s_{j} / 2} \leq \Theta(S) \frac{2^{(n-1+\|S\|) / 2} e^{-\|S\| / 2}}{r^{\|S\|}}(n-1+\|S\|)^{\|S\| / 2} \prod_{j=1}^{n}\left(\frac{e}{2 s_{j}}\right)^{s_{j} / 2}=
\end{gathered}
$$




$$
\begin{gathered}
=\Theta(S) \frac{2^{(n-1) / 2}}{r\|S\|}\left(1+\frac{n-1}{\|S\|}\right)^{\frac{\|S\|}{n-1} \cdot \frac{n-1}{2}} \cdot\|S\|^{\|S\| / 2} \prod_{j=1}^{n} \frac{1}{s_{j}^{s_{j} / 2}} \leq \\
\leq \Theta(S)(2 e)^{(n-1) / 2}\left(\frac{1}{r} \prod_{j=1}^{n}\left(\frac{\|S\|}{s_{j}}\right)^{\frac{s_{j}}{2\|S\|}}\right)^{\|S\|}, s_{j} \rightarrow \infty .
\end{gathered}
$$

Denote $x_{j}=\frac{\|S\|}{s_{j}} \in(1,+\infty), x=\left(x_{1}, \ldots, x_{n}\right)$. Obviously, $\Theta(S) \rightarrow 1$ as $s_{j} \rightarrow \infty, j \in$ $\{1, \ldots, n\}$. Then (53) implies a constrained optimization problem

$$
\begin{gathered}
H(x):=\prod_{j=1}^{n} x_{j}^{1 /\left(2 x_{j}\right)} \rightarrow \max \\
\text { subject to } \sum_{j=1}^{n} \frac{1}{x_{j}}=1, x_{j} \in(1,+\infty) .
\end{gathered}
$$

If this problem has a solution, then $H(x)$ is not greater than some $H^{*}$ and we choose $r>H^{*}$ in $(53)$.

Let us introduce a Lagrange multiplier $\lambda$ and study the Lagrange function $\mathcal{L}(x, \lambda)$ defined by

$$
\mathcal{L}(x, \lambda)=\prod_{j=1}^{n} x_{j}^{1 /\left(2 x_{j}\right)}+\lambda\left(\sum_{j=1}^{n} \frac{1}{x_{j}}-1\right) .
$$

A necessary condition for optimality in constrained problems yields that

$$
\frac{\partial \mathcal{L}}{\partial x_{j}}=\frac{1-\ln x_{j}}{2 x_{j}^{2}} \prod_{k=1}^{n} x_{k}^{1 /\left(2 x_{k}\right)}+\lambda\left(-\frac{1}{x_{j}^{2}}\right)=0
$$

or

$$
\frac{1-\ln x_{j}}{2}=\lambda / \prod_{k=1}^{n} x_{k}^{1 /\left(2 x_{k}\right)}
$$

Hence, $x_{j}=\exp \left(1-2 \lambda / \prod_{k=1}^{n} x_{k}^{1 /\left(2 x_{k}\right)}\right)$, i.e. $x_{1}=x_{2}=\ldots=x_{n}$. Constraint (54) implies that $\sum_{j=1}^{n} \frac{1}{x_{j}}=\frac{n}{x_{1}}=1$ or $x_{j}=n$ for every $j \in\{1, \ldots, n\}$. Then $H(x) \leq \prod_{j=1}^{n} n^{1 /(2 n)}=\sqrt{n}$.

We choose $r \geq \sqrt{n}$. For this $r$ we have $\frac{1}{r} \prod_{j=1}^{n}\left(\frac{\|S\|}{s_{j}}\right)^{\frac{s_{j}}{2\|S\|}} \leq 1$. In view of (53) it means that there exist $n_{2}$ such that

$$
\frac{(n+\|S\|-1) ! \prod_{j=1}^{n} \Gamma\left(s_{j} / 2+1\right)}{(K+S) ! \Gamma(n+\|S\| / 2) r\|S\|} \leq 1
$$

for all $\|S\| \geq n_{2}$.

The asymptotic behavior of right part (51) in other cases $S$ can be investigated similarly. Taking into account (51), (52) and (55) we have that for all $\|S\| \geq n_{1}+n_{2}$

$$
\frac{\left|F^{(K+S)}\left(z^{0}\right)\right|}{(K+S) ! \mathbf{L}^{S+K}\left(z^{0}\right)} \leq \frac{\left|F^{\left(K^{0}\right)}\left(z^{0}\right)\right|}{K^{0} ! \mathbf{L}^{K^{0}}\left(z^{0}\right)} .
$$


This means that for every $J \in \mathbb{Z}_{+}^{n}$

$$
\frac{\left|F^{(J)}\left(z^{0}\right)\right|}{J ! \mathbf{L}^{J}\left(z^{0}\right)} \leq \max \left\{\frac{\left|F^{(K)}\left(z^{0}\right)\right|}{K ! \mathbf{L}^{K}\left(z^{0}\right)}:\|K\| \leq n_{0}+n_{1}+n_{2}\right\}
$$

where $n_{0}, n_{1}, n_{2}$ are independent of $z_{0}$. Therefore, the function $F$ has bounded $\mathbf{L}$-index in joint variables with $N\left(F, \mathbf{L}, \mathbb{B}^{n}\right) \leq n_{0}+n_{1}+n_{2}$.

If we impose additional constraint by the function $\mathbf{L}$ then Theorem 12 implies the following criterion

Theorem 13. Let $\mathbf{L} \in Q^{\prime}\left(\mathbb{B}^{n}\right)$ be such that $\sup _{z \in \mathbb{B}^{n}} \frac{\mathcal{L}(z)}{\ell(z)}=C<\infty$. An analytic function $F$ in $\mathbb{B}^{n}$ has bounded L-index in joint variables if and only if for each $r \in(0, \beta]$ there exist $n_{0} \in \mathbb{Z}_{+}, p_{0}>0$ such that for every $z^{0} \in \mathbb{B}^{n}$ there exists $K^{0} \in \mathbb{Z}_{+}^{n},\left\|K^{0}\right\| \leq n_{0}$, such that inequality (42) holds.

Proof. Sufficiency is proved in Theorem 12. As for necessity we choose $q=q(R)=[2(N+$ 1) $\left.C r \prod_{j=1}^{n}\left(\lambda_{1, j}(r)\right)^{-N}\left(\lambda_{2, j}(r)\right)^{N+1}\right]+1$ and replace $\mathcal{L}\left(z^{0}\right)$ by $\ell\left(z^{0}\right)$ in the proof of Theorem 12 . No other changes.

Theorem 14. Let $\mathbf{L} \in Q^{\prime}\left(\mathbb{B}^{n}\right)$. In order that an analytic function $F$ in $\mathbb{B}^{n}$ be of bounded L-index in joint variables it is necessary that for every $r \in(0, \beta] \exists n_{0} \in \mathbb{Z}_{+} \exists p \geq 1 \forall z^{0} \in \mathbb{B}^{n}$ $\exists K^{0} \in \mathbb{Z}_{+}^{n},\left\|K^{0}\right\| \leq n_{0}$, and

$$
\max \left\{\left|F^{\left(K^{0}\right)}(z)\right|: z \in \mathbb{B}^{n}\left[z^{0}, r / \mathcal{L}\left(z^{0}\right)\right]\right\} \leq p\left|F^{\left(K^{0}\right)}\left(z^{0}\right)\right|
$$

and it is sufficient that for every $r \in(0, \beta]$

$$
\exists n_{0} \in \mathbb{Z}_{+} \exists p \geq 1 \forall z^{0} \in \mathbb{B}^{n} \quad \forall j \in\{1, \ldots, n\} \exists K_{j}^{0}=(0, \ldots, 0, \underbrace{k_{j}^{0}}_{j \text {-th place }}, 0, \ldots, 0)
$$

such that $k_{j}^{0} \leq n_{0}$ and

$$
\max \left\{\left|F^{\left(K_{j}^{0}\right)}(z)\right|: z \in \mathbb{B}^{n}\left[z^{0}, r / \ell\left(z^{0}\right)\right]\right\} \leq p\left|F^{\left(K_{j}^{0}\right)}\left(z^{0}\right)\right| \forall j \in\{1, \ldots, n\},
$$

Proof. Proof of Theorem 12 implies that the inequality (41) is true for some $K^{0}$. Therefore, we have

$$
\begin{aligned}
& \frac{p_{0}}{K^{0} !} \frac{\left|F^{\left(K^{0}\right)}\left(z^{0}\right)\right|}{\mathbf{L}^{K^{0}}\left(z^{0}\right)} \geq \max \left\{\frac{\left|F^{\left(K^{0}\right)}(z)\right|}{K^{0} ! \mathbf{L}^{K^{0}}(z)}: z \in \mathbb{B}^{n}\left[z^{0}, r / \mathcal{L}\left(z^{0}\right)\right]\right\}= \\
& =\max \left\{\frac{\left|F^{\left(K^{0}\right)}(z)\right|}{K^{0} !} \frac{\mathbf{L}^{K^{0}}\left(z^{0}\right)}{\mathbf{L}^{K^{0}}\left(z^{0}\right) \mathbf{L}^{K^{0}}(z)}: z \in \mathbb{B}^{n}\left[z^{0}, r / \mathcal{L}\left(z^{0}\right)\right]\right\} \geq \\
& \geq \max \left\{\frac{\left|F^{\left(K^{0}\right)}(z)\right|}{K^{0 !}} \frac{\prod_{j=1}^{n}\left(\lambda_{2, j}(r)\right)^{-n_{0}}}{\mathbf{L}^{K^{0}}\left(z^{0}\right)}: z \in \mathbb{B}^{n}\left[z^{0}, r / \mathcal{L}\left(z^{0}\right)\right]\right\} .
\end{aligned}
$$

This inequality implies

$$
\frac{p_{0} \prod_{j=1}^{n}\left(\lambda_{2, j}(r)\right)^{n_{0}}}{K^{0} !} \frac{\left|F^{\left(K^{0}\right)}\left(z^{0}\right)\right|}{\mathbf{L}^{K^{0}}\left(z^{0}\right)} \geq \max \left\{\frac{\left|F^{\left(K^{0}\right)}(z)\right|}{K^{0} ! \mathbf{L}^{K^{0}}\left(z^{0}\right)}: z \in \mathbb{B}^{n}\left[z^{0}, r / \mathcal{L}\left(z^{0}\right)\right]\right\} .
$$


From (58) we obtain inequality (56) with $p=p_{0} \prod_{j=1}^{n}\left(\lambda_{2, j}(r)\right)^{n_{0}}$. The necessity of condition (56) is proved.

Now we prove the sufficiency of (57). Suppose that for every $r \in(0, \beta] \exists n_{0} \in \mathbb{Z}_{+}, p>1$ such that $\forall z_{0} \in \mathbb{B}^{n}$ and some $K_{j}^{0} \in \mathbb{Z}_{+}^{n}$ with $k_{j}^{0} \leq n_{0}$ the inequality (57) holds.

$$
\frac{F^{\left(K_{J}^{0}+S\right)}\left(z^{0}\right)}{S !}=\frac{1}{(2 \pi i)^{2}} \int_{T^{n}\left(z^{0}, R / \mathbf{L}\left(z^{0}\right)\right)} \frac{F^{\left(K_{J}^{0}\right)}(z)}{\left(z-z^{0}\right)^{S+\mathbf{e}}} d z .
$$

In view of (49) we write Cauchy's formula as following $\forall z^{0} \in \mathbb{B}^{n} \forall S \in \mathbb{Z}_{+}^{n}$

$$
F^{\left(K_{j}^{0}+S\right)}\left(z^{0}\right)=\frac{(n+\|S\|-1) !}{(n-1) !} \int_{\mathbb{S}^{n}\left(z^{0}, r / \ell\left(z^{0}\right)\right)} \frac{\left(\overline{\xi-z^{0}}\right)^{S} F^{\left(K_{j}^{0}\right)}(\xi)}{\left|\xi-z^{0}\right|^{2(n+\|S\|)-1}} d \sigma(\xi)
$$

As in (50), this yields

$$
\begin{aligned}
& \leq \frac{(n+\|S\|-1) !}{(n-1) !}\left(\frac{\ell\left(z^{0}\right)}{r}\right)^{2(n+\|S\|)-1} \max \left\{\left|F^{\left(K_{j}^{0}+S\right)}(z)\right|: z \in \mathbb{B}^{n}\left[z^{0}, r / \ell\left(z^{0}\right)\right]\right\} \times \\
& \times \int_{\mathbb{S}_{n}\left(z^{0}, r / \ell\left(z^{0}\right)\right)}\left|\left(\overline{\xi-z^{0}}\right)^{S}\right| d \sigma(\xi) \leq \frac{(n+\|S\|-1) !}{(n-1) !}\left(\frac{\ell\left(z^{0}\right)}{r}\right)^{\|S\|} \times \\
& \times \max \left\{\left|F^{\left(K_{j}^{0}\right)}(z)\right|: z \in \mathbb{B}^{n}\left[z^{0}, r / \ell\left(z^{0}\right)\right]\right\} \int_{\mathbb{S}^{n}\left(z^{0}, r / \ell\left(z^{0}\right)\right)} \frac{\left|\left(\xi-z^{0}\right)^{S}\right|}{\left(r / \ell\left(z^{0}\right)\right)^{|| S \|}} d \sigma\left(\frac{\xi-z^{0}}{r / \ell\left(z^{0}\right)}\right) \leq \\
& \leq \frac{(n+\|S\|-1) !}{(n-1) !}\left(\frac{\ell\left(z^{0}\right)}{r}\right)^{\|S\|} \max \left\{\left|F^{\left(K_{j}^{0}\right)}(z)\right|: z \in \mathbb{B}^{n}\left[z^{0}, r / \ell\left(z^{0}\right)\right]\right\} \times \\
& \times \int_{\mathbb{S}^{n}(\mathbf{0}, 1)}\left|\xi^{S}\right| d \sigma(\xi)=\frac{(n+\|S\|-1) !}{(n-1) !}\left(\frac{\ell\left(z^{0}\right)}{r}\right)^{\|S\|} \times \\
& \times \max \left\{\left|F^{\left(K_{j}^{0}\right)}(z)\right|: z \in \mathbb{B}^{n}\left[z^{0}, r / \ell\left(z^{0}\right)\right]\right\} \frac{\Gamma(n) \prod_{j=1}^{n} \Gamma\left(s_{j} / 2+1\right)}{\Gamma(n+\|S\| / 2)} .
\end{aligned}
$$

Now we put $r=\beta$ and use (57)

$$
\begin{aligned}
& \left|F^{\left(K_{j}^{0}+S\right)}\left(z^{0}\right)\right| \leq\left(\frac{\ell\left(z^{0}\right)}{\beta}\right)^{\|S\|} \frac{(n+\|S\|-1) ! \prod_{j=1}^{n} \Gamma\left(s_{j} / 2+1\right)}{\Gamma(n+\|S\| / 2)} \times \\
& \times \max \left\{\left|F^{\left(K_{j}^{0}\right)}(z)\right|: z \in \mathbb{B}^{n}\left[z^{0}, \beta / \ell\left(z^{0}\right)\right]\right\} \leq \\
& \leq p\left(\frac{\ell\left(z^{0}\right)}{\beta}\right)^{\|S\|} \frac{(n+\|S\|-1) ! \prod_{j=1}^{n} \Gamma\left(s_{j} / 2+1\right)}{\Gamma(n+\|S\| / 2)}\left|F^{\left(K_{j}^{0}\right)}\left(z^{0}\right)\right| .
\end{aligned}
$$

Therefore (59) implies for all $j \in\{1, \ldots, n\}$ and $k_{j}^{0} \leq n_{0}$

$$
\frac{\left|F^{\left(K_{j}^{0}+S\right)}\left(z^{0}\right)\right|}{\mathbf{L}^{K_{j}^{0}+S}\left(z^{0}\right)\left(K_{j}^{0}+S\right) !} \leq p \frac{K_{j}^{0} !(n+\|S\|-1) ! \prod_{j=1}^{n} \Gamma\left(s_{j} / 2+1\right)}{\beta^{\|S\|}\left(K_{j}^{0}+S\right) ! \Gamma(n+\|S\| / 2)} \frac{\left|F^{\left(K_{j}^{0}\right)}\left(z^{0}\right)\right|}{\mathbf{L}^{K_{j}^{0}}\left(z^{0}\right) K_{j}^{0} !} \leq
$$




$$
\leq p n_{0} ! \frac{(n+\|S\|-1) ! \prod_{j=1}^{n} \Gamma\left(s_{j} / 2+1\right)}{\beta\|S\| S ! \Gamma(n+\|S\| / 2)} \frac{\left|F^{\left(K_{j}^{0}\right)}\left(z^{0}\right)\right|}{\mathbf{L}^{K_{j}^{0}}\left(z^{0}\right) K_{j}^{0 !}} .
$$

In view of (55) there exists $n_{1}$ such that for all $\|S\| \geq n_{1}$

$$
\frac{(n+\|S\|-1) ! \prod_{j=1}^{n} \Gamma\left(s_{j} / 2+1\right)}{\beta^{\|S\|} S ! \Gamma(n+\|S\| / 2)} \leq 1 .
$$

Obviously, there exists $n_{2}$ such that for all $\|S\| \geq n_{2} \frac{p n_{0} !}{\beta\|S\|} \leq 1$. Consequently, we have

$$
\frac{\left|F^{\left(K_{j}^{0}+S\right)}\left(z^{0}\right)\right|}{\mathbf{L}^{K_{j}^{0}+S}\left(z^{0}\right)\left(K_{j}^{0}+S\right) !} \leq \frac{\left|F^{\left(K_{j}^{0}\right)}\left(z^{0}\right)\right|}{\mathbf{L}^{K_{j}^{0}}\left(z^{0}\right) K_{j}^{0 !}} \text { for all }\|S\| \geq n_{1}+n_{2}
$$

i. e. $N\left(F, \mathbf{L}, \mathbb{B}^{n}\right) \leq n_{0}+n_{1}+n_{2}$.

Lemma 2. Let $\mathbf{L}_{1}, \mathbf{L}_{2}$ be positive continuous functions in $\mathbb{B}^{n}$ and for every $z \in \mathbb{B}^{n} \mathbf{L}_{1}(z) \leq$ $\mathbf{L}_{2}(z)$. If an analytic function $F$ in $\mathbb{B}^{n}$ has bounded $\mathbf{L}_{1}$-index in joint variables then $F$ is of bounded $\mathbf{L}_{2}$-index in joint variables. If, in addition, for every $z \in \mathbb{B}^{n} \mathcal{L}_{1}(z) \leq \ell_{2}(z)$ then $N\left(F, \mathbf{L}_{2}, \mathbb{B}^{n}\right) \leq N\left(F, \mathbf{L}_{1}, \mathbb{B}^{n}\right)$.

Proof. Let $N\left(F, \mathbf{L}_{1}, \mathbb{B}^{n}\right)=n_{0}$. Using (2) we deduce

$$
\begin{aligned}
\frac{\left|F^{(J)}(z)\right|}{J ! \mathbf{L}_{2}^{J}(z)} & =\frac{\mathbf{L}_{1}^{J}(z)}{\mathbf{L}_{2}^{J}(z)} \frac{\left|F^{(J)}(z)\right|}{J ! \mathbf{L}_{1}^{J}(z)} \leq \frac{\mathbf{L}_{1}^{J}(z)}{\mathbf{L}_{2}^{J}(z)} \max \left\{\frac{\left|F^{(K)}(z)\right|}{K ! \mathbf{L}_{1}^{K}(z)}: \quad K \in \mathbb{Z}_{+}^{n},\|K\| \leq n_{0}\right\} \leq \\
& \leq \frac{\mathbf{L}_{1}^{J}(z)}{\mathbf{L}_{2}^{J}(z)} \max \left\{\frac{\mathbf{L}_{2}^{K}(z)}{\mathbf{L}_{1}^{K}(z)} \frac{\left|F^{(K)}(z)\right|}{K ! \mathbf{L}_{2}^{K}(z)}: K \in \mathbb{Z}_{+}^{n},\|K\| \leq n_{0}\right\} \leq \\
& \leq \max _{\|K\| \leq n_{0}}\left(\frac{\mathbf{L}_{1}(z)}{\mathbf{L}_{2}(z)}\right)^{J-K} \max \left\{\frac{\left|F^{(K)}(z)\right|}{K ! \mathbf{L}_{2}^{K}(z)}: K \in \mathbb{Z}_{+}^{n},\|K\| \leq n_{0}\right\} .
\end{aligned}
$$

Since $\mathbf{L}_{1}(z) \leq \mathbf{L}_{2}(z)$ it means that for all $\|J\| \geq n n_{0}$

$$
\frac{\left|F^{(J)}(z)\right|}{J ! \mathbf{L}_{2}^{J}(z)} \leq \max \left\{\frac{\left|F^{(K)}(z)\right|}{K ! \mathbf{L}_{2}^{K}(z)}: K \in \mathbb{Z}_{+}^{n},\|K\| \leq n_{0}\right\} .
$$

Thus, $F$ has bounded $\mathbf{L}_{2}$-index in joint variables.

If, in addition, for every $z \in \mathbb{B}^{n} \mathcal{L}_{1}(z) \leq \ell_{2}(z)$ then for all $\|J\| \geq n_{0}$ (60) yields

$$
\begin{gathered}
\frac{\left|F^{(J)}(z)\right|}{J ! \mathbf{L}_{2}^{J}(z)} \leq \max _{\|K\| \leq n_{0}}\left(\frac{\mathcal{L}_{1}(z)}{\ell_{2}(z)}\right)^{\|J-K\|} \max \left\{\frac{\left|F^{(K)}(z)\right|}{K ! \mathbf{L}_{2}^{K}(z)}: K \in \mathbb{Z}_{+}^{n},\|K\| \leq n_{0}\right\} \leq \\
\leq \max \left\{\frac{\left|F^{(K)}(z)\right|}{K ! \mathbf{L}_{2}^{K}(z)}: K \in \mathbb{Z}_{+}^{n},\|K\| \leq n_{0}\right\}
\end{gathered}
$$

and $N\left(F, \mathbf{L}_{2}, \mathbb{B}^{n}\right) \leq N\left(F, \mathbf{L}_{1}, \mathbb{B}^{n}\right)$.

Denote $\widetilde{\mathbf{L}}(z)=\left(\widetilde{l}_{1}(z), \ldots, \widetilde{l}_{n}(z)\right)$. The notation $\mathbf{L} \asymp \widetilde{\mathbf{L}}$ means that there exist $\Theta_{1}=$ $\left(\theta_{1, j}, \ldots, \theta_{1, n}\right) \in \mathbb{R}_{+}^{n}, \Theta_{2}=\left(\theta_{2, j}, \ldots, \theta_{2, n}\right) \in \mathbb{R}_{+}^{n}$ such that $\forall z \in \mathbb{B}^{n} \theta_{1, j} \widetilde{l}_{j}(z) \leq l_{j}(z) \leq \theta_{2, j} \widetilde{l}_{j}(z)$ for each $j \in\{1, \ldots, n\}$. 
Theorem 15. Let $\mathbf{L} \in Q^{\prime}\left(\mathbb{B}^{n}\right), \mathbf{L} \asymp \widetilde{\mathbf{L}}, \sup _{z \in \mathbb{B}^{n}} \frac{\mathcal{L}(z)}{\ell(z)}=C<\infty, \min _{1 \leq j \leq n} \theta_{1, j}>\frac{\sqrt{n}}{\beta}$. An analytic function $F$ in $\mathbb{B}^{n}$ has bounded $\widetilde{\mathbf{L}}$-index in joint variables if and only if $F$ has bounded L-index.

Proof. It is easy to prove that if $\mathbf{L} \in Q^{\prime}\left(\mathbb{B}^{n}\right)$ and $\mathbf{L} \asymp \widetilde{\mathbf{L}}$ then $\widetilde{\mathbf{L}} \in Q^{\prime}\left(\mathbb{B}^{n}\right)$ with $\beta^{\prime}=$ $\beta \min _{1 \leq j \leq n} \theta_{1, j}>\sqrt{n}$ instead of $\beta$ in (1).

Let $N\left(F, \widetilde{\mathbf{L}}, \mathbb{B}^{n}\right)=\widetilde{n}_{0}<+\infty$. Then by Theorem 12 for every $\widetilde{r} \in(0, \beta)$ there exists $\widetilde{p} \geq 1$ such that for each $z^{0} \in \mathbb{B}^{n}$ and some $K^{0}$ with $\left\|K^{0}\right\| \leq \widetilde{n}_{0}$, the inequality (41) holds with $\widetilde{\mathbf{L}}$ and $\widetilde{r}$ instead of $\mathbf{L}$ and $r$. Hence,

$$
\begin{gathered}
\quad \frac{\widetilde{p}}{K^{0} !} \frac{\left|F^{\left(K^{0}\right)}\left(z^{0}\right)\right|}{\mathbf{L}^{K^{0}}\left(z^{0}\right)}=\frac{\widetilde{p}}{K^{0} !} \frac{\Theta_{2}^{K^{0}}\left|F^{\left(K^{0}\right)}\left(z^{0}\right)\right|}{\Theta_{2}^{K^{0}} \mathbf{L}^{K^{0}}\left(z^{0}\right)} \geq \frac{\widetilde{p}}{K^{0} !} \frac{\left|F^{\left(K^{0}\right)}\left(z^{0}\right)\right|}{\Theta_{2}^{K^{0}} \widetilde{\mathbf{L}^{K^{0}}\left(z^{0}\right)} \geq} \\
\geq \frac{1}{\Theta_{2}^{K^{0}}} \max \left\{\frac{\left|F^{(K)}(z)\right|}{K ! \widetilde{\mathbf{L}}(z)}:\|K\| \leq \widetilde{n}_{0}, z \in \mathbb{B}^{n}\left[z^{0}, \widetilde{r} / \widetilde{\mathcal{L}}(z)\right]\right\} \geq \\
\geq \frac{1}{\Theta_{2}^{K^{0}}} \max \left\{\frac{\Theta_{1}^{K}\left|F^{(K)}(z)\right|}{K ! \mathbf{L}^{K}(z)}:\|K\| \leq \widetilde{n}_{0}, z \in \mathbb{B}^{n}\left[z^{0}, \min _{1 \leq j \leq n} \Theta_{1, j} \widetilde{r} / \mathcal{L}(z)\right]\right\} \geq \\
\geq \frac{\min _{0 \leq \|}\left\{\Theta_{1}^{K}\right\}}{\Theta_{2}^{K^{0}}} \max \left\{\frac{\left|F^{(K)}(z)\right|}{K ! \mathbf{L}^{K}(z)}:\|K\| \leq \widetilde{n}_{0}, z \in \mathbb{B}^{n}\left[z^{0}, \min _{1 \leq j \leq n} \Theta_{1, j} \widetilde{r} / \mathcal{L}(z)\right]\right\} \geq \\
\geq \frac{0 \leq\|K\| \leq n_{0}}{\Theta_{2}^{K^{0}}}\left\{\Theta_{1}^{K}\right\} \\
\max \left\{\frac{\left|F^{(K)}(z)\right|}{K ! \mathbf{L}^{K}(z)}:\|K\| \leq \widetilde{n}_{0}, z \in \mathbb{B}^{n}\left[z^{0}, \frac{\left.\widetilde{r} \min _{1 \leq j \leq n} \Theta_{1, j}\right]}{C \ell(z)}\right]\right\} .
\end{gathered}
$$

In view of Theorem 12, we obtain that function $F$ has bounded $\mathbf{L}$-index.

Theorem 16. Let $\mathbf{L} \in Q^{\prime}\left(\mathbb{B}^{n}\right)$, a function $F$ be analytic in $\mathbb{B}^{n}$. If there exist $r \in(0, \beta]$, $n_{0} \in \mathbb{Z}_{+}, p_{0}>1$ such that for each $z^{0} \in \mathbb{B}^{n}$ and for some $K^{0} \in \mathbb{Z}_{+}^{n}$ with $\left\|K^{0}\right\| \leq n_{0}$ the inequality (42) holds then $F$ has bounded $\mathbf{L}$-index in joint variables.

Proof. The proof of sufficiency in Theorem 12 for $r=\beta$ implies that $N\left(F, \mathbf{L}, \mathbb{B}^{n}\right)<+\infty$.

Let $\mathbf{L}^{*}(z)=\frac{r_{0} \mathbf{L}(z)}{r}, \ell^{*}(z)=\frac{r_{0} \ell(z)}{r}, r^{0}=\beta$ and $r$ is radius for which (42) is true. In a general case from validity of (42) for $F$ and $\mathbf{L}$ for $r<\beta$ we obtain

$$
\begin{gathered}
\max \left\{\frac{\left|F^{(K)}(z)\right|}{K !\left(\mathbf{L}^{*}(z)\right)^{K}}:\|K\| \leq n_{0}, z \in \mathbb{B}^{n}\left[z^{0}, r_{0} / \ell^{*}\left(z^{0}\right)\right]\right\} \leq \\
\leq \max \left\{\frac{\left|F^{(K)}(z)\right|}{K !\left(r_{0} \mathbf{L}(z) / r\right)^{K}}:\|K\| \leq n_{0}, z \in \mathbb{B}^{n}\left[z^{0}, r_{0} /\left(r_{0} \ell\left(z^{0}\right) / r\right)\right]\right\} \leq \\
\leq \max \left\{\frac{\left|F^{(K)}(z)\right|}{K ! \mathbf{L}^{K}(z)}:\|K\| \leq n_{0}, z \in \mathbb{B}^{n}\left[z^{0}, r / \ell\left(z^{0}\right)\right]\right\} \leq \\
\leq \frac{p_{0}}{K^{0} !} \frac{\left|F^{\left(K^{0}\right)}\left(z^{0}\right)\right|}{\mathbf{L}^{K^{0}}\left(z^{0}\right)}=\frac{\beta^{\left\|K^{0}\right\|} p_{0}}{r^{\left\|K^{0}\right\|} K^{0 !} !} \frac{\left|F^{\left(K^{0}\right)}(z)\right|}{\left(r_{0} \mathbf{L}(z) / r\right)^{K^{0}}}=\frac{p_{0} \beta^{n_{0}}}{r^{n_{0}}} \frac{\left|F^{\left(K^{0}\right)}(z)\right|}{K^{0} !\left(\mathbf{L}^{*}(z)\right)^{K^{0}}} .
\end{gathered}
$$

i. e. (41) holds for $F, \mathbf{L}^{*}$ and $r_{0}=\beta$. As above now we apply Theorem 12 to the function $F(z)$ and $\mathbf{L}^{*}(z)=r_{0} \mathbf{L}(z) / r$. This implies that $F$ is of bounded $\mathbf{L}^{*}$-index in joint variables. Therefore, by Lemma 2 the function $F$ has bounded L-index in joint variables.

8. Boundedness of $L$-index in joint variables of analytic solutions of systems of partial differential equations. Using Theorems 11 and 1 we obtain this corollary. 
Corollary 5. Let $\mathbf{L} \in Q^{n}, F(z)$ be an analytic function in $\mathbb{B}^{n}, G$ be a bounded domain in $\mathbb{B}^{n}$ such that $d=\inf _{z \in \bar{G}}(1-|z|)>0$. The function $F(z)$ is of bounded $\mathbf{L}$-index in joint variables and only if there exist $p \in \mathbb{Z}_{+}$and $C>0$ such that for all $z \in \mathbb{B}^{n} \backslash G$ the inequality (8) holds.

In one-dimensional case, this corollary was obtained in [33]. It was proposed to apply the corollary in investigation of index boundedness for entire solutions of linear higher order differential equations ([20]).

Let us denote $a^{+}=\max \{a, 0\}, u_{j}(t)=u_{j}(t, R, \Theta)=l_{j}\left(\frac{t R}{r^{*}} e^{i \Theta}\right)$, where $a \in \mathbb{R}, t \in\left[0, r^{*}\right]$, $j \in\{1, \ldots, n\}, r^{*}=\max _{1 \leq j \leq n} r_{j} \neq 0$ that is $\frac{t}{r^{*}}|R|<1$.

Let $\mathbf{L}\left(R e^{i \Theta}\right)$ be a positive continuously differentiable function in each variable $r_{k}, k \in$ $\{1, \ldots, n\},|R|<1, \Theta \in[0,2 \pi]^{n}$. By $W\left(\mathbb{B}^{n}\right)$ we denote the class of the functions $\mathbf{L}$ such that

$$
r^{*}\left(-\left(u_{j}(t, R, \Theta)\right)_{t=r^{*}}^{\prime}\right)^{+} /\left(r_{j} l_{j}^{2}\left(R e^{i \Theta}\right)\right) \rightarrow 0
$$

uniformly in $\Theta \in[0,2 \pi]^{n}, j \in\{1, \ldots, n\}$, as $|R| \rightarrow 1-0, W:=W^{1}$.

It is easy to check that condition (61) can be replaced by the stronger restriction

$$
\frac{\left\langle z, \nabla l_{j}(z)\right\rangle}{\left|z_{j}\right| l_{j}^{2}(z)} \rightarrow 0
$$

as $|z| \rightarrow 1-0$.

Lemma 3. If $\mathbf{L}(z)=\left(l_{1}(z), \ldots, l_{n}(z)\right)$, where every $l_{j}(z): \mathbb{B}^{n} \rightarrow \mathbb{R}_{+}$is a continuous function satisfying (1) then

$$
\max _{\Theta \in[0,2 \pi]^{n}} \int_{0}^{1}\left\langle R, \mathbf{L}\left(\tau R e^{i \Theta}\right)\right\rangle d \tau \rightarrow+\infty \text { as }|R| \rightarrow 1-0 .
$$

Proof. Using (1) we obtain

$$
\begin{gathered}
\max _{\Theta \in[0,2 \pi]^{n}} \int_{0}^{r^{*}} \sum_{j=1}^{n} \frac{r_{j}}{r^{*}} l_{j}\left(\frac{\tau}{r^{*}} R e^{i \Theta}\right) d \tau \geq \int_{0}^{r^{*}} \sum_{j=1}^{n} \frac{r_{j}}{r^{*}} \frac{\beta}{1-\frac{\tau}{r^{*}}|R|} d \tau= \\
=-\sum_{j=1}^{n} \frac{r_{j} \beta}{R} \ln (1-|R|) \rightarrow+\infty \text { as }|R| \rightarrow 1-0 .
\end{gathered}
$$

Lemma 4. Let $\mathbf{L} \in W\left(\mathbb{B}^{n}\right), F$ be an analytic function in $\mathbb{B}^{n}$. If there exists $R^{\prime} \in \mathbb{R}_{+}^{n}$, $\left|R^{\prime}\right|<1$, and $p \in \mathbb{Z}_{+}, c>0$ such that for all $z \in \mathbb{B}^{n} \backslash \mathbb{D}^{n}\left(\mathbf{0}, R^{\prime}\right)$ inequality (8) holds then

$$
\varlimsup_{|R| \rightarrow 1-0} \frac{\ln \max \left\{|F(z)|: z \in \mathbb{T}^{n}(\mathbf{0}, R)\right\}}{\max _{\Theta \in[0,2 \pi]^{n}} \int_{0}^{1}\left\langle R, \mathbf{L}\left(\tau R e^{i \Theta}\right)\right\rangle d \tau} \leq c .
$$

Proof. Let $R \in \mathbb{R}_{+}^{n}$ be such that $1>|R|>\left|R^{\prime}\right|, \Theta \in[0,2 \pi]^{n}$. Denote $\alpha_{j}=\frac{r_{j}}{r^{*}}, j \in\{1, \ldots, n\}$ and $A=\left(\alpha_{1}, \ldots, \alpha_{n}\right)$. We consider the function

$$
g(t)=\max \left\{\frac{\left|F^{(S)}\left(A t e^{i \Theta}\right)\right|}{\mathbf{L}^{S}\left(A t e^{i \Theta}\right)}:\|S\| \leq p\right\}
$$


where $A t e^{i \Theta}=\left(\alpha_{1} t e^{i \theta_{1}}, \ldots, \alpha_{n} t e^{i \theta_{n}}\right)$ and $|A t|>\left|R^{\prime}\right|$.

Since the function $\frac{\left|F^{(S)}\left(A t e^{i \Theta}\right)\right|}{\mathbf{L}^{S}\left(A t e^{i \Theta}\right)}$ is continuously differentiable by real $t \in\left[0, r^{*}\right]$, outside the zero set of function $\left|F^{(S)}\left(A t e^{i \Theta}\right)\right|$, the function $g(t)$ is a continuously differentiable function on $\left[0, r^{*}\right]$, except, perhaps, for a countable set of points.

Therefore, using the inequality $\frac{d}{d r}|g(r)| \leq\left|g^{\prime}(r)\right|$ which holds except for the points $r=t$ such that $g(t)=0$, we deduce

$$
\begin{gathered}
\frac{d}{d t}\left(\frac{\left|F^{(S)}\left(A t e^{i \Theta}\right)\right|}{\mathbf{L}^{S}\left(A t e^{i \Theta}\right)}\right)=\frac{1}{\mathbf{L}^{S}\left(A t e^{i \Theta}\right)} \frac{d}{d t}\left|F^{(S)}\left(A t e^{i \Theta}\right)\right|+ \\
+\left|F^{(S)}\left(A t e^{i \Theta}\right)\right| \frac{d}{d t} \frac{1}{\mathbf{L}^{S}\left(A t e^{i \Theta}\right)} \leq \frac{1}{\mathbf{L}^{S}\left(A t e^{i \Theta}\right)}\left|\sum_{j=1}^{n} F^{\left(S+\mathbf{e}_{j}\right)}\left(A t e^{i \Theta}\right) \alpha_{j} e^{i \theta_{j}}\right|- \\
-\frac{\left|F^{(S)}\left(A t e^{i \Theta}\right)\right|}{\mathbf{L}^{S}\left(A t e^{i \Theta}\right)} \sum_{j=1}^{n} \frac{s_{j} u_{j}^{\prime}(t)}{l_{j}\left(A t e^{i \Theta}\right)} \leq \sum_{j=1}^{n} \frac{\left|F^{\left(S+\mathbf{e}_{j}\right)}\left(A t e^{i \Theta}\right)\right|}{\mathbf{L}^{S+\mathbf{e}_{j}}\left(A t e^{i \Theta}\right)} \alpha_{j} l_{j}\left(A t e^{i \Theta}\right)+ \\
+\frac{\left|F^{(S)}\left(A t e^{i \Theta}\right)\right|}{\mathbf{L}^{S}\left(A t e^{i \Theta}\right)} \sum_{j=1}^{n} \frac{s_{j}\left(-u_{j}^{\prime}(t)\right)^{+}}{l_{j}\left(A t e^{i \Theta}\right)}
\end{gathered}
$$

For absolutely continuous functions $h_{1}, h_{2}, \ldots, h_{k}$ and $h(x):=\max \left\{h_{j}(z): 1 \leq j \leq k\right\}$, $h^{\prime}(x) \leq \max \left\{h_{j}^{\prime}(x): 1 \leq j \leq k\right\}, x \in[a, b]$ (see [35, Lemma 4.1, p. 81]). The function $g$ is absolutely continuous, therefore, from (8) and (63) it follows that

$$
\begin{gathered}
g^{\prime}(t) \leq \max \left\{\frac{d}{d t}\left(\frac{\left|F^{(S)}\left(A t e^{i \Theta}\right)\right|}{\mathbf{L}^{S}\left(A t e^{i \Theta}\right)}\right):\|S\| \leq p\right\} \leq \\
\leq \max _{\|S\| \leq p}\left\{\sum_{j=1}^{n} \frac{\alpha_{j} l_{j}\left(A t e^{i \Theta}\right)\left|F^{\left(S+\mathbf{e}_{j}\right)}\left(A t e^{i \Theta}\right)\right|}{\mathbf{L}^{S+\mathbf{e}_{j}}\left(A t e^{i \Theta}\right)}+\frac{\left|F^{(S)}\left(A t e^{i \Theta}\right)\right|}{\mathbf{L}^{S}\left(A t e^{i \Theta}\right)} \sum_{j=1}^{n} \frac{s_{j}\left(-u_{j}^{\prime}(t)\right)^{+}}{l_{j}\left(A t e^{i \Theta}\right)}\right\} \leq \\
\leq g(t)\left(\max \{1, c\} \sum_{j=1}^{n} \alpha_{j} l_{j}\left(A t e^{i \Theta}\right)+\max _{\|S\| \leq p}\left\{\sum_{j=1}^{n} \frac{s_{j}\left(-u_{j}^{\prime}(t)\right)^{+}}{l_{j}\left(A t e^{i \Theta}\right)}\right\}\right)=g(t)(\beta(t)+\gamma(t)),
\end{gathered}
$$

where

$$
\beta(t)=\max \{1, c\} \sum_{j=1}^{n} \alpha_{j} l_{j}\left(A t e^{i \Theta}\right), \gamma(t)=\max _{\|S\| \leq p}\left\{\sum_{j=1}^{n} \frac{s_{j}\left(-u_{j}^{\prime}(t)\right)^{+}}{l_{j}\left(A t e^{i \Theta}\right)}\right\} .
$$

Thus, $\frac{d}{d t} \ln g(t) \leq \beta(t)+\gamma(t)$ and

$$
g(t) \leq g\left(t_{0}\right) \exp \int_{t_{0}}^{t}(\beta(\tau)+\gamma(\tau)) d \tau
$$

where $t_{0}$ is chosen such that $g\left(t_{0}\right) \neq 0$. The condition $\mathbf{L} \in W\left(\mathbb{B}^{n}\right)$ gives

$$
\frac{\gamma(t)}{\beta(t)}=\frac{\sum_{j=1}^{n} \frac{s_{j}\left(-u_{j}^{\prime}(t)\right)^{+}}{l_{j}\left(A t e^{i \Theta}\right)}}{c \sum_{j=1}^{n} \alpha_{j} l_{j}\left(A t e^{i \Theta}\right)} \leq p \sum_{j=1}^{n} \frac{\left(-u_{j}^{\prime}(t)\right)^{+}}{\alpha_{j} l_{j}^{2}\left(A t e^{i \Theta}\right)} \leq p \varepsilon,
$$

where $\varepsilon=\varepsilon(R) \rightarrow 0$ uniformly in $\Theta \in[0,2 \pi]^{n}, t=r^{*}$ as $|R| \rightarrow 1-0$. 
But $\left|F\left(A t e^{i \Theta}\right)\right| \leq g(t) \leq g\left(t_{0}\right) \exp \int_{t_{0}}^{t}(\beta(\tau)+\gamma(\tau)) d \tau$ and $r^{*} A=R$. Then we put $t=r^{*}$ and obtain

$$
\begin{gathered}
\ln \max \left\{\mid F(z): z \in \mathbb{T}^{n}(\mathbf{0}, R)\right\}=\ln \max _{\Theta \in[0,2 \pi]^{n}}\left|F\left(R e^{i \Theta}\right)\right| \leq \ln \max _{\Theta \in[0,2 \pi]^{n}} g\left(r^{*}\right) \leq \\
\leq \ln g\left(t_{0}\right)+\max _{\Theta \in[0,2 \pi]^{n}} \int_{t_{0}}^{r^{*}}(\beta(\tau)+\gamma(\tau)) d \tau \leq \\
\leq \ln g\left(t_{0}\right)+\max _{\Theta \in[0,2 \pi]^{n}} \int_{t_{0}}^{r^{*}} c \sum_{j=1}^{n} \alpha_{j} l_{j}\left(A \tau e^{i \Theta}\right)(1+p \varepsilon) d \tau= \\
=\ln g\left(t_{0}\right)+\max \{1, c\} \max _{\Theta \in[0,2 \pi]^{n}} \int_{t_{0}}^{r^{*}} \sum_{j=1}^{n} \frac{r_{j}}{r^{*}} l_{j}\left(\frac{\tau}{r^{*}} R e^{i \Theta}\right)(1+p \varepsilon) d \tau .
\end{gathered}
$$

This implies (62).

Lemma 5. Let $\mathbf{L} \in W\left(\mathbb{B}^{n}\right), F$ be an analytic function in $\mathbb{B}^{n}$. If there exists $R^{\prime} \in \mathbb{R}_{+}^{n}$, $\left|R^{\prime}\right|<1$ and $p \in \mathbb{Z}_{+}, c>0$ such that for all $z \in \mathbb{B}^{n} \backslash \mathbb{D}^{n}\left(\mathbf{0}, R^{\prime}\right)$ inequality

$$
\max \left\{\frac{\left|F^{(J)}(z)\right|}{J ! \mathbf{L}^{J}(z)}:\|J\|=p+1\right\} \leq c \cdot \max \left\{\frac{\left|F^{(K)}(z)\right|}{K ! \mathbf{L}^{K}(z)}:\|K\| \leq p\right\}
$$

holds then

$$
\varlimsup_{|R| \rightarrow 1-0} \frac{\ln \max \left\{|F(z)|: z \in \mathbb{T}^{n}(\mathbf{0}, R)\right\}}{\max _{\Theta \in[0,2 \pi]^{n}} \int_{0}^{1}\left\langle R, \mathbf{L}\left(\tau R e^{i \Theta}\right)\right\rangle d \tau} \leq(p+1) \max \{1, c\} .
$$

Proof. The proof of Lemma 5 is similar to that of Lemma 4.

Let $R \in \mathbb{R}_{+}^{n}$ be such that $1>|R|>\left|R^{\prime}\right|, \Theta \in[0,2 \pi]^{n}$. Denote $\alpha_{j}=\frac{r_{j}}{r^{*}}, j \in\{1, \ldots, n\}$ and $A=\left(\alpha_{1}, \ldots, \alpha_{n}\right)$. We consider the function

$$
g(t)=\max \left\{\frac{\left|F^{(S)}\left(A t e^{i \Theta}\right)\right|}{S ! \mathbf{L}^{S}\left(A t e^{i \Theta}\right)}:\|S\| \leq p\right\},
$$

where $A t=\left(\alpha_{1} t, \ldots, \alpha_{n} t\right), A t e^{i \Theta}=\left(\alpha_{1} t e^{i \theta_{1}}, \ldots, \alpha_{n} t e^{i \theta_{n}}\right)$ and $|A t|>\left|R^{\prime}\right|$.

As above the function $\frac{\left|F^{(S)}\left(A t e^{i \Theta}\right)\right|}{S ! \mathbf{L}^{S}\left(A t e^{i \Theta}\right)}$ is continuously differentiable by real $t \in\left[0, r^{*}\right]$, outside the zero set of the function $\left|F^{(S)}\left(A t e^{i \Theta}\right)\right|$, the function $g(t)$ is a continuously differentiable function on $\left[0, r^{*}\right]$, except, perhaps, for a countable set of points.

Therefore, using the inequality $\frac{d}{d r}|g(r)| \leq\left|g^{\prime}(r)\right|$ which holds except for the points $r=t$ such that $g(t)=0$, we deduce

$$
\begin{aligned}
\frac{d}{d t}\left(\frac{\left|F^{(S)}\left(A t e^{i \Theta}\right)\right|}{S ! \mathbf{L}^{S}\left(A t e^{i \Theta}\right)}\right) & =\frac{1}{S ! \mathbf{L}^{S}\left(A t e^{i \Theta}\right)} \frac{d}{d t}\left|F^{(S)}\left(A t e^{i \Theta}\right)\right|+ \\
+\left|F^{(S)}\left(A t e^{i \Theta}\right)\right| \frac{d}{d t} \frac{1}{S ! \mathbf{L}^{S}\left(A t e^{i \Theta}\right)} & \leq \frac{1}{S ! \mathbf{L}^{S}\left(A t e^{i \Theta}\right)}\left|\sum_{j=1}^{n} F^{\left(S+\mathbf{e}_{j}\right)}\left(A t e^{i \Theta}\right) \alpha_{j} e^{i \theta_{j}}\right|-
\end{aligned}
$$




$$
\begin{gathered}
-\frac{\left|F^{(S)}\left(A t e^{i \Theta}\right)\right|}{S ! \mathbf{L}^{S}\left(A t e^{i \Theta}\right)} \sum_{j=1}^{n} \frac{s_{j} u_{j}^{\prime}(t)}{l_{j}\left(A t e^{i \Theta}\right)} \leq \sum_{j=1}^{n} \frac{\left|F^{\left(S+\mathbf{e}_{j}\right)}\left(A t e^{i \Theta}\right)\right|}{\left(S+\mathbf{e}_{j}\right) ! \mathbf{L}^{S+\mathbf{e}_{j}}\left(A t e^{i \Theta}\right)} \alpha_{j}\left(s_{j}+1\right) l_{j}\left(A t e^{i \Theta}\right)+ \\
+\frac{\left|F^{(S)}\left(A t e^{i \Theta}\right)\right|}{S ! \mathbf{L}^{S}\left(A t e^{i \Theta}\right)} \sum_{j=1}^{n} \frac{s_{j}\left(-u_{j}^{\prime}(t)\right)^{+}}{l_{j}\left(A t e^{i \Theta}\right)} .
\end{gathered}
$$

For absolutely continuous functions $h_{1}, h_{2}, \ldots, h_{k}$ and $h(x):=\max \left\{h_{j}(z): 1 \leq j \leq k\right\}$, $h^{\prime}(x) \leq \max \left\{h_{j}^{\prime}(x): 1 \leq j \leq k\right\}, x \in[a, b]$ (see [35, Lemma 4.1, p. 81]). The function $g$ is absolutely continuous. Therefore, (8) and (66) yield

$$
\begin{gathered}
g^{\prime}(t) \leq \max \left\{\frac{d}{d t}\left(\frac{\left|F^{(S)}\left(A t e^{i \Theta}\right)\right|}{S ! \mathbf{L}^{S}\left(A t e^{i \Theta}\right)}\right):\|S\| \leq N\right\} \leq \\
\leq \max _{\|S\| \leq p}\left\{\sum_{j=1}^{n} \frac{\alpha_{j}\left(s_{j}+1\right) l_{j}\left(A t e^{i \Theta}\right)\left|F^{\left(S+\mathbf{e}_{j}\right)}\left(A t e^{i \Theta}\right)\right|}{\left(S+\mathbf{e}_{j}\right) ! \mathbf{L}^{S+\mathbf{e}_{j}}\left(A t e^{i \Theta}\right)}+\frac{\left|F^{(S)}\left(A t e^{i \Theta}\right)\right|}{S ! \mathbf{L}^{S}\left(A t e^{i \Theta}\right)} \sum_{j=1}^{n} \frac{s_{j}\left(-u_{j}^{\prime}(t)\right)^{+}}{l_{j}\left(A t e^{i \Theta}\right)}\right\} \leq \\
\leq g(t)\left(\max \{1, c\} \max _{\|S\| \leq p}\left\{\sum_{j=1}^{n} \alpha_{j}\left(s_{j}+1\right) l_{j}\left(A t e^{i \Theta}\right)\right\}+\right. \\
\left.+\max _{\|S\| \leq p}\left\{\sum_{j=1}^{n} \frac{s_{j}\left(-u_{j}^{\prime}(t)\right)^{+}}{l_{j}\left(A t e^{i \Theta}\right)}\right\}\right)=g(t)(\beta(t)+\gamma(t)),
\end{gathered}
$$

where

$$
\beta(t)=\max \{1, c\} \max _{\|S\| \leq p}\left\{\sum_{j=1}^{n} \alpha_{j}\left(s_{j}+1\right) l_{j}\left(A t e^{i \Theta}\right)\right\}, \gamma(t)=\max _{\|S\| \leq p}\left\{\sum_{j=1}^{n} \frac{s_{j}\left(-u_{j}^{\prime}(t)\right)^{+}}{l_{j}\left(A t e^{i \Theta}\right)}\right\} .
$$

Thus, $\frac{d}{d t} \ln g(t) \leq \beta(t)+\gamma(t)$ and

$$
g(t) \leq g\left(t_{0}\right) \exp \int_{t_{0}}^{t}(\beta(\tau)+\gamma(\tau)) d \tau
$$

where $t_{0}$ is chosen such that $g\left(t_{0}\right) \neq 0$. Denote $\widetilde{\beta}(t)=\sum_{j=1}^{n} \alpha_{j} l_{j}\left(A t e^{i \Theta}\right)$. Since $\mathbf{L} \in W\left(\mathbb{B}^{n}\right)$, for some $S^{*},\left\|S^{*}\right\| \leq p$ and $\widetilde{S},\|\widetilde{S}\| \leq p$, we obtain

$$
\begin{gathered}
\frac{\gamma(t)}{\widetilde{\beta}(t)}=\frac{\sum_{j=1}^{n} \frac{s_{j}^{*}\left(-u_{j}^{\prime}(t)\right)^{+}}{l_{j}\left(A t e^{i \Theta}\right)}}{\sum_{j=1}^{n} \alpha_{j} l_{j}\left(A t e^{i \Theta}\right)} \leq \sum_{j=1}^{n} s_{j}^{*} \frac{\left(-u_{j}^{\prime}(t)\right)^{+}}{\alpha_{j} l_{j}^{2}\left(A t e^{i \Theta}\right)} \leq p \sum_{j=1}^{n} \frac{\left(-u_{j}^{\prime}(t)\right)^{+}}{\alpha_{j} l_{j}^{2}\left(A t e^{i \Theta}\right)} \leq p \varepsilon \\
\frac{\beta(t)}{\widetilde{\beta}(t)}=\frac{\max \{1, c\} \sum_{j=1}^{n} \alpha_{j}\left(\tilde{s}_{j}+1\right) l_{j}\left(A t e^{i \Theta}\right)}{\sum_{j=1}^{n} \alpha_{j} l_{j}\left(A t e^{i \Theta}\right)}= \\
=\max \{1, c\}+\max \{1, c\} \frac{\sum_{j=1}^{n} \alpha_{j} \tilde{s}_{j} l_{j}\left(A t e^{i \Theta}\right)}{\sum_{j=1}^{n} \alpha_{j} l_{j}\left(A t e^{i \Theta}\right)} \leq \\
\leq \max \{1, c\}+\max \{1, c\} \sum_{j=1}^{n} \tilde{s}_{j} \leq \max \{1, c\}(1+p)
\end{gathered}
$$

where $\varepsilon=\varepsilon(R) \rightarrow 0$ uniformly in $\Theta \in[0,2 \pi]^{n}, t=r^{*}$ as $|R| \rightarrow 1-0$ 
But $\left|F\left(A t e^{i \Theta}\right)\right| \leq g(t) \leq g\left(t_{0}\right) \exp \int_{t_{0}}^{t}(\beta(\tau)+\gamma(\tau)) d \tau$ and $r^{*} A=R$. Then we put $t=r^{*}$ and obtain

$$
\begin{gathered}
\ln \max \left\{\mid F(z): \quad z \in \mathbb{T}^{n}(\mathbf{0}, R)\right\}=\ln \max _{\Theta \in[0,2 \pi]^{n}}\left|F\left(R e^{i \Theta}\right)\right| \leq \ln \max _{\Theta \in[0,2 \pi]^{n}} g\left(r^{*}\right) \leq \\
\leq \ln g\left(t_{0}\right)+\max _{\Theta \in[0,2 \pi]^{n}} \int_{t_{0}}^{r^{*}}(\beta(\tau)+\gamma(\tau)) d \tau \leq \\
\leq \ln g\left(t_{0}\right)+\max _{\Theta \in[0,2 \pi]^{n}} \int_{t_{0}}^{r^{*}} \sum_{j=1}^{n} \alpha_{j} l_{j}\left(A \tau e^{i \Theta}\right)(\max \{1, c\}(1+p)+p \varepsilon) d \tau= \\
=\ln g\left(t_{0}\right)+\max _{\Theta \in[0,2 \pi]^{n}} \int_{t_{0}} \sum_{j=1}^{n} \frac{r_{j}}{r^{*}} l_{j}\left(\frac{\tau}{r^{*}} R e^{i \Theta}\right)(\max \{1, c\}(1+p)+p \varepsilon) d \tau .
\end{gathered}
$$

This implies (65).

Using proved lemmas we will formulate and prove propositions that provide growth estimates for analytic solutions of the following system of partial differential equations:

$$
G_{p_{j} \mathbf{e}_{j}}(z) F^{\left(p_{j} \mathbf{e}_{j}\right)}(z)+\sum_{\left\|S_{j}\right\| \leq p_{j}-1} G_{S_{j}}(z) F^{\left(S_{j}\right)}(z)=H_{j}(z), j \in\{1, \ldots, n\}
$$

$p_{j} \in \mathbb{N}, S_{j} \in \mathbb{Z}_{+}^{n}, H_{j}$ and $G_{S_{j}}$ are analytic functions in $\mathbb{B}^{n}$. Denote $Q W\left(\mathbb{B}^{n}\right)=Q\left(\mathbb{B}^{n}\right) \cap W\left(\mathbb{B}^{n}\right)$. Accordingly, $Q W(\mathbb{D})=Q(\mathbb{D}) \cap W(\mathbb{D})$.

We will say that non-homogeneous system of PDE's (67) belongs to class $\mathcal{A}(\mathbf{G}, \mathbf{H}, \mathbf{L})$, if $\mathbf{L} \in Q W\left(\mathbb{B}^{n}\right)$, for all $z \in \mathbb{B}^{n}$ and for every $j \in\{1, \ldots, n\}$ the analytic functions $H_{j}$ and $G_{S_{j}}$ in $\mathbb{B}^{n}$ satisfy the following conditions:

1) for every $\left\|S_{j}\right\| \leq p_{j}-1$ and for each $M \in \mathbb{Z}_{+}^{n}$,

$$
\begin{gathered}
\|M\| \leq 1+\sum_{\substack{k=1 \\
k \neq j}}^{n} p_{k},\left|G_{S_{j}}^{(M)}(z)\right| \mathbf{L}^{S_{j}-M}(z) \leq B_{S_{j}, M} l_{j}^{p_{j}}(z)\left|G_{p_{j} \mathbf{1}_{j}}(z)\right| \\
\left|G_{p_{j} \mathbf{1}_{j}}^{(M)}(z)\right| \leq B_{p_{j} \mathbf{1}_{j}, M} \mathbf{L}^{M}(z)\left|G_{p_{j} \mathbf{1}_{j}}(z)\right|
\end{gathered}
$$

2) for every $I \in \mathbb{Z}_{+}^{n}$,

$$
\|I\|=1+\sum_{\substack{k=1 \\ k \neq j}}^{n} p_{k}\left|H_{j}^{(I)}(z)\right| \leq D_{I, j} \mathbf{L}^{I}(z)\left|H_{j}(z)\right|,
$$

3) $G_{p_{j} \mathbf{1}_{j}}(z) \neq 0$, where $B_{S_{j}, M}, D_{I, j}, B_{p_{j} \mathbf{1}_{j}, M}$ are positive constants, $\mathbf{H}(z)=\left(H_{1}(z), \ldots\right.$, $\left.H_{n}(z)\right), \mathbf{G}(z)$ is a matrix consisting of coefficients $G_{S_{J}}(z)$ of system (67).

Homogeneous system of PDE's (67) belongs to class $\mathcal{A}(\mathbf{G}, \mathbf{0}, \mathbf{L})$, if condition 1) holds for $M \in \mathbb{Z}_{+}^{n}$, such that $\|M\| \leq \sum_{\substack{k=1 \\ k \neq j}}^{n} p_{k}$ and $G_{p_{j} \mathbf{1}_{j}}(z) \neq 0$. Conditon 2) is not required in this case. 
Instead of $G_{p_{j} \mathbf{1}_{j}}(z) \neq 0$ we can require validity of conditions 1) and 2) for all $z \in$ $\mathbb{B}^{n} \backslash \mathbb{D}^{n}\left(\mathbf{0}, R^{\prime}\right)$. It is possible in view of Theorem 11 . If for some $M \in \mathbb{Z}_{+}^{n} G_{S_{j}}^{(M)}(z) \equiv 0$ or $H_{j}^{(M)}(z) \equiv 0$ then we suppose that $B_{S_{j}, M}=0$ or $D_{M, j}=0$, respectively.

Theorem 17. If non-homogeneous system of PDE's (67) belongs to class $\mathcal{A}(\mathbf{G}, \mathbf{H}, \mathbf{L})$ and an analytic function $F(z)$ in $\mathbb{B}^{n}$ satisfies (67) then $F$ has bounded $\mathbf{L}$-index in joint variables and

$$
\varlimsup_{|R| \rightarrow 1-0} \frac{\ln \max \left\{|F(z)|: z \in \mathbb{T}^{n}(\mathbf{0}, R)\right\}}{\max _{\Theta \in[0,2 \pi]^{n}} \int_{0}^{1}\left\langle R, \mathbf{L}\left(\tau R e^{i \Theta}\right)\right\rangle d \tau} \leq \max \{1, c\},
$$

where $c$ is defined in (73).

Proof. Taking into account that the function $F(z)$ satisfies system (67), we calculate the partial derivative $I \in \mathbb{Z}_{+}^{n}$ in each equation of the system

$$
\begin{gathered}
\sum_{\mathbf{0} \leq M \leq I} C_{I}^{M} G_{p_{j} \mathbf{e}_{j}}^{(M)}(z) F^{\left(p_{j} \mathbf{e}_{j}+I-M\right)}(z)+ \\
+\sum_{\mathbf{0} \leq M \leq I} C_{I}^{M} \sum_{\left\|S_{j}\right\| \leq p_{j}-1} G_{S_{j}}^{(M)}(z) F^{\left(S_{j}+I-M\right)}(z)=H_{j}^{(I)}(z),
\end{gathered}
$$

where $C_{I}^{M}=\frac{i_{1} ! \ldots i_{n} !}{m_{1} !\left(i_{1}-m_{1}\right) ! \ldots . m_{n} !\left(i_{n}-m_{n}\right) !}$ and

$$
\|I\|=1-p_{j}+\sum_{k=1}^{n} p_{k}=1+\sum_{\substack{k=1 \\ k \neq j}}^{n} p_{k} .
$$

Using the second condition of the class definition $\mathcal{A}(\mathbf{G}, \mathbf{H}, \mathbf{L})$, we obtain

$$
\begin{gathered}
\left|H_{j}^{(I)}(z)\right| \leq D_{I, j} \mathbf{L}^{I}(z)\left|H_{j}(z)\right| \leq \\
\leq D_{I, j} \mathbf{L}^{I}(z)\left(\left|G_{p_{j} \mathbf{e}_{j}}(z)\right|\left|F^{\left(p_{j} \mathbf{e}_{j}\right)}(z)\right|+\sum_{\left\|S_{j}\right\| \leq p_{j}-1}\left|G_{S_{j}}(z) \| F^{\left(S_{j}\right)}(z)\right|\right) .
\end{gathered}
$$

Equation (69) yields

$$
\begin{aligned}
F^{\left(p_{j} \mathbf{e}_{j}+I\right)}(z) & =\frac{1}{G_{p_{j} \mathbf{e}_{j}}(z)}\left(H_{j}^{(I)}(z)-\sum_{\substack{\mathbf{0} \leq M \leq I \\
M \neq \mathbf{0}}} C_{I}^{M} G_{p_{j} \mathbf{e}_{j}}^{(M)}(z) F^{\left(p_{j} \mathbf{e}_{j}+I-M\right)}(z)-\right. \\
& \left.-\sum_{\mathbf{0} \leq M \leq I} C_{I}^{M} \sum_{\left\|S_{j}\right\| \leq p_{j}-1} G_{S_{j}}^{(M)}(z) F^{\left(S_{j}+I-M\right)}(z)\right) .
\end{aligned}
$$

From (71) and the first condition it follows

$$
\begin{aligned}
& \left|F^{\left(p_{j} \mathbf{e}_{j}+I\right)}(z)\right|=\frac{1}{\left|G_{p_{j} \mathbf{e}_{j}}(z)\right|}\left(D_{I, j} \mathbf{L}^{I}(z)\left(\left|G_{p_{j} \mathbf{e}_{j}}(z)\right|\left|F^{\left(p_{j} \mathbf{e}_{j}\right)}(z)\right|+\sum_{\left\|S_{j}\right\| \leq p_{j}-1}\left|G_{S_{j}}(z) \| F^{\left(S_{j}\right)}(z)\right|\right)+\right. \\
& \left.\quad+\sum_{\substack{\mathbf{0} \leq M \leq I \\
M \neq \mathbf{0}}} C_{I}^{M}\left|G_{p_{j} \mathbf{e}_{j}}^{(M)}(z)\right|\left|F^{\left(p_{j} \mathbf{e}_{j}+I-M\right)}(z)\right|+\sum_{\mathbf{0} \leq M \leq I} C_{I}^{M} \sum_{\left\|S_{j}\right\| \leq p_{j}-1}\left|G_{S_{j}}^{(M)}(z)\right|\left|F^{\left(S_{j}+I-M\right)}(z)\right|\right) \leq
\end{aligned}
$$




$$
\begin{aligned}
\leq D_{I, j} \mathbf{L}^{I}(z) & \left(\left|F^{\left(p_{j} \mathbf{e}_{j}\right)}(z)\right|+\sum_{\left\|S_{j}\right\| \leq p_{j}-1} B_{S_{j}, \mathbf{0}} L^{p_{j} \mathbf{e}_{j}-S_{j}}(z)\left|F^{\left(S_{j}\right)}(z)\right|\right)+ \\
& +\sum_{\substack{\mathbf{0} \leq M \leq I \\
M \neq \mathbf{0}}} C_{I}^{M} B_{p_{j} \mathbf{e}_{j}, M} \mathbf{L}^{M}(z)\left|F^{\left(p_{j} \mathbf{e}_{j}+I-M\right)}(z)\right|+ \\
& +\sum_{\mathbf{0} \leq M \leq I} C_{I}^{M} \sum_{\left\|S_{j}\right\| \leq p_{j}-1} B_{S_{j}, M} \mathbf{L}^{p_{j} \mathbf{e}_{j}-S_{j}+M}(z)\left|F^{\left(S_{j}+I-M\right)}(z)\right| .
\end{aligned}
$$

Dividing this inequality by $L^{p_{j} \mathbf{e}_{j}+I}(z)$, we obtain that for every

$$
\|I\|=1+\sum_{\substack{k=1 \\ k \neq j}}^{n} p_{k}
$$

and $j \in\{1, \ldots, n\}$

$$
\begin{gathered}
\frac{\left|F^{\left(p_{j} \mathbf{e}_{j}+I\right)}(z)\right|}{\mathbf{L}^{p_{j} \mathbf{e}_{j}+I}(z)} \leq D_{I, j}\left(\frac{\left|F^{\left(p_{j} \mathbf{e}_{j}\right)}(z)\right|}{\mathbf{L}^{p_{j} \mathbf{e}_{j}}(z)}+\sum_{\left\|S_{j}\right\| \leq p_{j}-1} B_{S_{j}, \mathbf{0}} \frac{\left|F^{\left(S_{j}\right)}(z)\right|}{\mathbf{L}^{S_{j}}(z)}\right)+ \\
+\sum_{\substack{\mathbf{0} \leq M \leq I \\
M \neq \mathbf{0}}} C_{I}^{M} B_{p_{j} \mathbf{e}_{j}, M} \frac{\left|F^{\left(p_{j} \mathbf{e}_{j}+I-M\right)}(z)\right|}{\mathbf{L}^{p_{j} \mathbf{e}_{j}+I-M}(z)}+\sum_{\mathbf{0} \leq M \leq I} C_{I}^{M} \sum_{\left\|S_{j}\right\| \leq p_{j}-1} B_{S_{j}, M} \frac{\left|F^{\left(S_{j}+I-M\right)}(z)\right|}{\mathbf{L}^{S_{j}+I-M}(z)} \leq \\
\leq\left(D_{I, j}\left(1+\sum_{\left\|S_{j}\right\| \leq p_{j}-1} B_{S_{j}, \mathbf{0}}\right)+\sum_{\substack{\mathbf{0} \leq M \leq I \\
M \neq \mathbf{0}}} C_{I}^{M} B_{p_{j} \mathbf{e}_{j}, M}+\sum_{\mathbf{0} \leq M \leq I} C_{I}^{M} \sum_{\left\|S_{j}\right\| \leq p_{j}-1} B_{S_{j}, M}\right) \times \\
\times \max \left\{\frac{\left|F^{(S)}(z)\right|}{\mathbf{L}^{S}(z)}:\|S\| \leq \sum_{j=1}^{n} p_{j}\right\} .
\end{gathered}
$$

Obviously, $\left\|p_{j} \mathbf{e}_{j}+I\right\|=1+\sum_{j=1}^{n} p_{j}$. This implies

$$
\max \left\{\frac{\left|F^{(K)}(z)\right|}{\mathbf{L}^{K}(z)}:\|K\|=1+\sum_{j=1}^{n} p_{j}\right\} \leq \max \{1, c\} \cdot \max \left\{\frac{\left|F^{(S)}(z)\right|}{\mathbf{L}^{S}(z)}:\|S\| \leq \sum_{j=1}^{n} p_{j}\right\},
$$

where

$$
\begin{aligned}
c=\max _{\substack{\|I\|=1-p_{j}+\sum_{k=1}^{n} p_{k} \\
j \in\{1, \ldots, n\}}} & \left(D_{I, j}\left(1+\sum_{\left\|S_{j}\right\| \leq p_{j}-1} B_{S_{j}, \mathbf{0}}\right)+\sum_{\substack{\mathbf{0} \leq M \leq I \\
M \neq \mathbf{0}}} C_{I}^{M} B_{p_{j} \mathbf{e}_{j}, M}+\right. \\
& \left.+\sum_{\mathbf{0} \leq M \leq I} C_{I}^{M} \sum_{\left\|S_{j}\right\| \leq p_{j}-1} B_{S_{j}, M}\right)
\end{aligned}
$$

for all $z \in \mathbb{C}^{n} \backslash \mathbb{D}^{n}\left(\mathbf{0}, R^{\prime}\right)$.

Thus, by Lemma 4 estimate (68) holds, and by Corollary 5 the analytic function $F$ in $\mathbb{B}^{n}$ has bounded $\mathbf{L}$-index in joint variables. 
If system $(67)$ is homogeneous $\left(H_{j}(z) \equiv 0\right)$, the previous theorem can be simplified.

Theorem 18. If homogeneous system of PDE's (67) belongs to class $\mathcal{A}(\mathbf{G}, \mathbf{0}, \mathbf{L})$ and an analytic function $F$ in $\mathbb{B}^{n}$ is a solution of the system then $F$ has bounded $\mathbf{L}$-index in joint variables and

$$
\varlimsup_{|R| \rightarrow 1-0} \frac{\ln \max \left\{|F(z)|: z \in \mathbb{T}^{n}(\mathbf{0}, R)\right\}}{\max _{\Theta \in[0,2 \pi]^{n}} \int_{0}^{1}\left\langle R, \mathbf{L}\left(\tau R e^{i \Theta}\right)\right\rangle d \tau} \leq \max \{1, c\},
$$

where $c$ is defined in (73) with $D_{I, j}=0$ and $\|I\|=-p_{j}+\sum_{k=1}^{n} p_{k}$ instead of $\|I\|=$ $1-p_{j}+\sum_{k=1}^{n} p_{k}$.

Proof. If $H_{j}(z) \equiv 0$ then (71) implies

$$
\begin{gathered}
F^{\left(p_{j} \mathbf{e}_{j}+I\right)}(z)=\frac{1}{G_{p_{j} \mathbf{e}_{j}}(z)}\left(-\sum_{\substack{\mathbf{0} \leq M \leq I \\
M \neq \mathbf{0}}} C_{I}^{M} G_{p_{j} \mathbf{e}_{j}}^{(M)}(z) F^{\left(p_{j} \mathbf{e}_{j}+I-M\right)}(z)-\right. \\
\left.\quad-\sum_{\mathbf{0} \leq M \leq I} C_{I}^{M} \sum_{\left\|S_{j}\right\| \leq p_{j}-1} G_{S_{j}}^{(M)}(z) F^{\left(S_{j}+I-M\right)}(z)\right) .
\end{gathered}
$$

Hence, we obtain

$$
\begin{gathered}
\left|F^{\left(p_{j} \mathbf{e}_{j}+I\right)}(z)\right| \leq \frac{1}{\left|G_{p_{j} \mathbf{e}_{j}}(z)\right|}\left(\sum_{\substack{\mathbf{0} M M \leq I \\
M \neq 0}} C_{I}^{M}\left|G_{p_{j} \mathbf{e}_{j}}^{(M)}(z) \| F^{\left(p_{j} \mathbf{e}_{j}+I-M\right)}(z)\right|+\right. \\
\left.\quad+\sum_{\mathbf{0} \leq M \leq I} C_{I}^{M} \sum_{\left\|S_{j}\right\| \leq p_{j}-1}\left|G_{S_{j}}^{(M)}(z)\right|\left|F^{\left(S_{j}+I-M\right)}(z)\right|\right) .
\end{gathered}
$$

Dividing the obtained inequality by $\mathbf{L}^{p_{j} \mathbf{e}_{j}+I}(z)$ and using assumptions of the theorem on the functions $G_{S_{j}}$, we deduce

$$
\begin{gathered}
\frac{\left|F^{\left(p_{j} \mathbf{e}_{j}+I\right)}(z)\right|}{\mathbf{L}^{p_{j} \mathbf{e}_{j}+I}(z)} \leq \frac{1}{\left|G_{p_{j} \mathbf{e}_{j}}(z)\right| \mathbf{L}^{p_{j} \mathbf{e}_{j}+I}(z)}\left(\sum_{\substack{0 \leq M \leq I \\
M \neq \mathbf{0}}} C_{I}^{M} B_{p_{j} \mathbf{e}_{j}, M} \mathbf{L}^{M}(z)\left|G_{p_{j} \mathbf{e}_{j}}(z)\right|\left|F^{\left(p_{j} \mathbf{e}_{j}+I-M\right)}(z)\right|+\right. \\
\left.\quad+\sum_{\mathbf{0} \leq M \leq I} C_{I}^{M} \sum_{\left\|S_{j}\right\| \leq p_{j}-1} B_{S_{j}, M} \mathbf{L}^{p_{j} \mathbf{e}_{j}-S_{j}+M}(z)\left|G_{p_{j} \mathbf{e}_{j}}(z)\right|\left|F^{\left(S_{j}+I-M\right)}(z)\right|\right)= \\
=\sum_{\substack{\mathbf{0} \leq M \leq I \\
M \neq \mathbf{0}}} C_{I}^{M} B_{p_{j} \mathbf{e}_{j}, M} \frac{\left|F^{\left(p_{j} \mathbf{e}_{j}+I-M\right)}(z)\right|}{\mathbf{L}^{p_{j} \mathbf{e}_{j}+I-M}(z)}+\sum_{\mathbf{0} \leq M \leq I} C_{I}^{M} \sum_{\left\|S_{j}\right\| \leq p_{j}-1} B_{S_{j}, M} \frac{\left|F^{\left(S_{j}+I-M\right)}(z)\right|}{\mathbf{L}^{S_{j}+I-M}(z)} \leq \\
\leq\left(\sum_{\substack{\mathbf{0} \leq M \leq I \\
M \neq \mathbf{0}}} C_{I}^{M} B_{p_{j} \mathbf{e}_{j}, M}+\sum_{\mathbf{0} \leq M \leq I} C_{I}^{M} \sum_{\left\|S_{j}\right\| \leq p_{j}-1} B_{S_{j}, M}\right) \times
\end{gathered}
$$




$$
\times \max \left\{\frac{\left|F^{(S)}(z)\right|}{\mathbf{L}^{S}(z)}:\|S\| \leq-1+\sum_{j=1}^{n} p_{j}\right\} .
$$

Obviously, $\left\|p_{j} \mathbf{e}_{j}+I\right\|=\sum_{j=1}^{n} p_{j}$. Therefore,

$$
\max \left\{\frac{\left|F^{(K)}(z)\right|}{\mathbf{L}^{K}(z)}:\|K\|=\sum_{j=1}^{n} p_{j}\right\} \leq \max \{1, c\} \cdot \max \left\{\frac{\left|F^{(S)}(z)\right|}{\mathbf{L}^{S}(z)}:\|S\| \leq-1+\sum_{j=1}^{n} p_{j}\right\},
$$

where

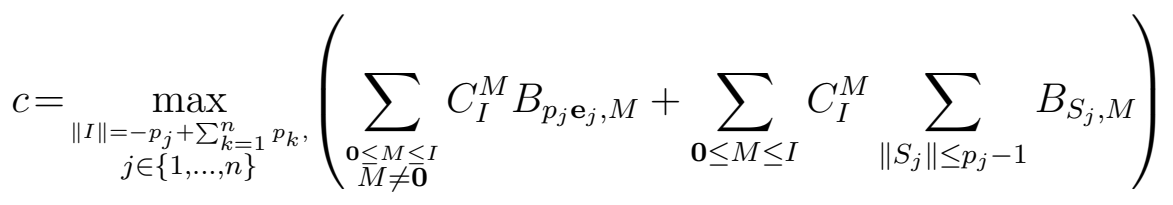

for all $z \in \mathbb{B}^{n} \backslash \mathbb{D}^{n}\left(\mathbf{0}, R^{\prime}\right)$.

Thus, all conditions of Corollary 5 are satisfying. Hence, the function $F$ has bounded L-index in joint variables and by Lemma 4 estimate (74) holds.

Note that estimate (68) and (74) cannot be improved (see examples for $n=1$ in [20]).

Moreover, using Corollary 5 and Lemma 5 we can supplement two previous Theorems 17 and 18 with propositions that contain estimates of $\max \left\{|F(z)|: z \in \mathbb{T}^{n}(\mathbf{0}, R)\right\}$, which can sometimes be better than (74) and (68). Two following theorems have proofs that of to Theorems 17 and 18.

Theorem 19. If non-homogeneous system of PDE's (67) belongs to class $\mathcal{A}(\mathbf{G}, \mathbf{H}, \mathbf{L})$ and an analytic function $F(z)$ in $\mathbb{B}^{n}$ satisfies (67) then $F$ has bounded $\mathbf{L}$-index in joint variables and

$$
\varlimsup_{|R| \rightarrow 1-0} \frac{\ln \max \left\{|F(z)|: z \in \mathbb{T}^{n}(\mathbf{0}, R)\right\}}{\max _{\Theta \in[0,2 \pi]^{n}} \int_{0}^{1}\left\langle R, \mathbf{L}\left(\tau R e^{i \Theta}\right)\right\rangle d \tau} \leq \max \{1, c\},
$$

where $c^{\prime}$ is defined in (77).

Proof. As in proof of Theorem 17, dividing (72) by $\left(p_{j} \mathbf{e}_{j}+I\right) ! L^{p_{j} \mathbf{e}_{j}+I}(z)$, we obtain that for every $\|I\|=1+\sum_{\substack{k=1 \\ k \neq j}}^{n} p_{k}$ and $j \in\{1, \ldots, n\}$

$$
\begin{aligned}
& \frac{\left|F^{\left(p_{j} \mathbf{e}_{j}+I\right)}(z)\right|}{\left(p_{j} \mathbf{e}_{j}+I\right) ! \mathbf{L}^{p_{j} \mathbf{e}_{j}+I}(z)} \leq D_{I, j}\left(\frac{\left|F^{\left(p_{j} \mathbf{e}_{j}\right)}(z)\right|}{\left(p_{j} \mathbf{e}_{j}+I\right) ! \mathbf{L}^{p_{j} \mathbf{e}_{j}}(z)}+\sum_{\left\|S_{j}\right\| \leq p_{j}-1} B_{S_{j}, \mathbf{0}} \frac{\left|F^{\left(S_{j}\right)}(z)\right|}{\left(p_{j} \mathbf{e}_{j}+I\right) ! \mathbf{L}^{p_{j} \mathbf{e}_{j}-S_{j}(z)}}\right)+ \\
&+\sum_{\substack{\mathbf{0} \leq M \leq I \\
M \neq \mathbf{0}}} C_{I}^{M} B_{p_{j} \mathbf{e}_{j}, M} \frac{\left|F^{\left(p_{j} \mathbf{e}_{j}+I-M\right)}(z)\right|}{\left(p_{j} \mathbf{e}_{j}+I\right) ! \mathbf{L}^{p_{j} \mathbf{e}_{j}+I-M}(z)}+ \\
&+\sum_{\mathbf{0} \leq M \leq I} C_{I}^{M} \sum_{\left\|S_{j}\right\| \leq p_{j}-1} B_{S_{j}, M} \frac{\left|F^{\left(S_{j}+I-M\right)}(z)\right|}{\left(p_{j} \mathbf{e}_{j}+I\right) ! \mathbf{L}^{S_{j}+I-M}(z)} \leq \\
& \leq D_{I, j}\left(\frac{\left|F^{\left(p_{j} \mathbf{e}_{j}\right)}(z)\right|}{\left(p_{j} \mathbf{e}_{j}+I\right) ! \mathbf{L}^{p_{j} \mathbf{e}_{j}}(z)}+B \sum_{\left\|S_{j}\right\| \leq p_{j}-1} \frac{\left|F^{\left(S_{j}\right)}(z)\right|}{\left(p_{j} \mathbf{e}_{j}+I\right) ! \mathbf{L}^{p_{j} \mathbf{e}_{j}-S_{j}}(z)}\right)+
\end{aligned}
$$




$$
\begin{aligned}
& +B \sum_{\substack{\mathbf{0} \leq M \leq I \\
M \neq \mathbf{0}}} C_{I}^{M} \frac{\left|F^{\left(p_{j} \mathbf{e}_{j}+I-M\right)}(z)\right|}{\left(p_{j} \mathbf{e}_{j}+I\right) ! \mathbf{L}^{p_{j} \mathbf{e}_{j}+I-M}(z)}+ \\
& +B \sum_{\mathbf{0} \leq M \leq I} C_{I}^{M} \sum_{\left\|S_{j}\right\| \leq p_{j}-1} \frac{\left|F^{\left(S_{j}+I-M\right)}(z)\right|}{\left(p_{j} \mathbf{e}_{j}+I\right) ! \mathbf{L}^{S_{j}+I-M}(z)} \leq \\
& \leq\left(D_{I, j}\left(\frac{p_{j} !}{\left(p_{j} \mathbf{e}_{j}+I\right) !}+B \sum_{\left\|S_{j}\right\| \leq p_{j}-1} \frac{\left(p_{j} \mathbf{e}_{j}-S_{j}\right) !}{\left(p_{j} \mathbf{e}_{j}+I\right) !}\right)+\right. \\
& \left.+B \sum_{\substack{\mathbf{0} \leq M \leq I \\
M \neq \mathbf{0}}} C_{I}^{M} \frac{\left(p_{j} \mathbf{e}_{j}+I-M\right) ! \mid}{\left(p_{j} \mathbf{e}_{j}+I\right) !}+B \sum_{\mathbf{0} \leq M \leq I} C_{I}^{M} \sum_{\left\|S_{j}\right\| \leq p_{j}-1} \frac{\left(S_{j}+I-M\right) !}{\left(p_{j} \mathbf{e}_{j}+I\right) !}\right) \times \\
& \times \max \left\{\frac{\left|F^{(S)}(z)\right|}{\mathbf{L}^{S}(z)}:\|S\| \leq \sum_{j=1}^{n} p_{j}\right\} .
\end{aligned}
$$

where $B=\max \left\{B_{S_{j}, M}, B_{p_{j} \mathbf{e}_{j}, M}: j \in\{1, \ldots, n\}, \mathbf{0} \leq M \leq I,\|I\|=1+\sum_{\substack{k=1 \\ k \neq j}}^{n} p_{k}.\right\}$

Obviously, $\left\|p_{j} \mathbf{e}_{j}+I\right\|=1+\sum_{j=1}^{n} p_{j}$. For all $z \in \mathbb{C}^{n} \backslash \mathbb{D}^{n}\left(\mathbf{0}, R^{\prime}\right)$ it implies

$$
\max \left\{\frac{\left|F^{(K)}(z)\right|}{K ! \mathbf{L}^{K}(z)}:\|K\|=1+\sum_{j=1}^{n} p_{j}\right\} \leq \max \left\{1, c^{\prime}\right\} \cdot \max \left\{\frac{\left|F^{(S)}(z)\right|}{S ! \mathbf{L}^{S}(z)}:\|S\| \leq \sum_{j=1}^{n} p_{j}\right\}
$$

where

$$
\begin{gathered}
c^{\prime}=\max _{\substack{\|I\|=1-p_{j}+\sum_{k=1}^{n} p_{k} \\
j \in\{1, \ldots, n\}}}\left(D_{I, j}\left(\frac{p_{j} !}{\left(p_{j} \mathbf{e}_{j}+I\right) !}+B \sum_{\left\|S_{j}\right\| \leq p_{j}-1} \frac{\left(p_{j} \mathbf{e}_{j}-S_{j}\right) !}{\left(p_{j} \mathbf{e}_{j}+I\right) !}\right)+\right. \\
\left.+B \sum_{\substack{\mathbf{0} \leq M \leq I \\
M \neq \mathbf{0}}} C_{I}^{M} \frac{\left(p_{j} \mathbf{e}_{j}+I-M\right) ! \mid}{\left(p_{j} \mathbf{e}_{j}+I\right) !}+B \sum_{\mathbf{0} \leq M \leq I} C_{I}^{M} \sum_{\left\|S_{j}\right\| \leq p_{j}-1} \frac{\left(S_{j}+I-M\right) !}{\left(p_{j} \mathbf{e}_{j}+I\right) !}\right) .
\end{gathered}
$$

In view of Corollary 5 the analytic function $F$ in $\mathbb{B}^{n}$ has bounded $\mathbf{L}$-index in joint variables. And by Lemma 5 estimate (76) holds.

By analogy to the proofs of Theorems 18 and 19 it can be proved the following assertion.

Theorem 20. If homogeneous system of PDE's (67) belongs to class $\mathcal{A}(\mathbf{G}, \mathbf{0}, \mathbf{L})$ and $F$ is an analytic solution of the system in $\mathbb{B}^{n}$ then $F$ has bounded $\mathbf{L}$-index in joint variables and

$$
\varlimsup_{|R| \rightarrow 1-0} \frac{\ln \max \left\{|F(z)|: z \in \mathbb{T}^{n}(\mathbf{0}, R)\right\}}{\max _{\Theta \in[0,2 \pi]^{n}} \int_{0}^{1}\left\langle R, \mathbf{L}\left(\tau R e^{i \Theta}\right)\right\rangle d \tau} \leq \max \left\{1, c^{\prime}\right\}
$$

where $c^{\prime}$ is defined in (77) with $D_{I, j}=0$ and $\|I\|=-p_{j}+\sum_{k=1}^{n} p_{k}$ instead $\|I\|=1-p_{j}+$ $\sum_{k=1}^{n} p_{k}$.

Remark 2. The obtained propositions in this section are new even for functions analytic in a disc. Analytic functions in the unit disc of bounded $l$-index are considered in $[35,36]$. 
For example, if $n=1$ then system (67) reduces to the following differential equation

$$
g_{p}(z) f^{(p)}(z)+\sum_{j-0}^{p-1} g_{j}(z) f^{(j)}(z)=h(z),
$$

where $h$ and $g_{j}$ are analytic functions in $\mathbb{D}$. Then Theorem 17 implies the corollary for $n=1$.

Corollary 6. Let $l \in Q W(\mathbb{D})$ and for all $z \in \mathbb{C}$ such that $|z|>r^{\prime}$ analytic functions $h$ and $g_{j}$ in $\mathbb{D}$ satisfy the following conditions

1) $\left|g_{j}^{(m)}(z)\right| \leq B_{j, m} l^{p-j+m}(z)\left|g_{p}(z)\right|$ and $\left|g_{p}^{\prime}(z)\right|<B_{p, 1} l^{m}(z)\left|g_{p}(z)\right|$ for every $j \in\{1, \ldots, p-$ $1\}, m \in\{0,1\}$,

2) $\left|h^{\prime}(z)\right| \leq D l(z)|h(z)|$,

where $B_{j, m}$ and $D$ are nonnegative constants, and $B_{p, 1}$ is positive constant. If an analytic function $f$ in $\mathbb{D}$ satisfies (78) then $f$ has bounded l-index and

$$
\varlimsup_{r \rightarrow 1-0} \frac{\ln \max \{|f(z)|:|z|=r\}}{\max _{\theta \in[0,2 \pi]} \int_{0}^{r} l\left(\tau e^{i \theta}\right) d \tau} \leq c,
$$

where $c=D\left(1+\sum_{j=0}^{p-1} B_{j, 0}\right)+B_{p, 1}+\sum_{m=0}^{1} \sum_{j=0}^{p-1} B_{j, m}$.

\section{REFERENCES}

1. A. Bandura, O. Skaskiv, Functions analytic in a unit ball of bounded L-index in joint variables, J. Math. Sci. 227 (2017), №1, 1-12.

2. A. Bandura, O. Skaskiv, Sufficient conditions of boundedness of L-index and analog of Hayman's Theorem for analytic functions in a ball, Studia Universitatis Babeş-Bolyai Mathematica (in print), avialable at https://arxiv.org/abs/1705.09568.

3. A.I. Bandura, O.B. Skaskiv, Entire functions of bounded L-index in direction, Mat. Stud., 27 (2007), №1, 30-52. (in Ukrainian)

4. A.I. Bandura, Sum of entire functions of bounded L-index in direction, Mat. Stud., 45 (2016), №2, $149-158$.

5. A. Bandura, O. Skaskiv, Entire functions of several variables of bounded index, Publisher I.E.Chyzhykov, Lviv, 2016.

6. A.I. Bandura, O.B. Skaskiv, Directional logarithmic derivative and the distribution of zeros of an entire function of bounded L-index along the direction, Ukrain. Mat. J., 69 (2017), №1, 500-508.

7. A. Bandura, O. Skaskiv, P. Filevych, Properties of entire solutions of some linear PDE's, J. Appl. Math. Comput. Mech., 16 (2017), №2, 17-28.

8. A. Bandura, O. Skaskiv, Analytic in the unit ball functions of bounded L-index in direction, (submitted in Rocky Mountain Journal of Mathematics), avialable at https://arxiv.org/abs/1501.04166.

9. A.I. Bandura, M.T. Bordulyak, O.B. Skaskiv, Sufficient conditions of boundedness of $\mathbf{L}$-index in joint variables, Mat. Stud., 45 (2016), №1, 12-26.

10. A. Bandura, New criteria of boundedness of $\mathbf{L}$-index in joint variables for entire functions, Math. Bull. Shevchenko Sci. Soc., 13 (2016), 58-67. (in Ukrainian)

11. A. Bandura, N. Petrechko, Properties of power series expansion of entire function of bounded $\mathbf{L}$-index in joint variables, Visn. Lviv Un-ty, Ser. Mech. Math., 82 (2016), 27-33. (in Ukrainian) 
12. A.I. Bandura, N.V. Petrechko, O.B. Skaskiv, Analytic functions in a polydisc of bounded $\mathbf{L}$-index in joint variables, Mat. Stud., 46 (2016), №1, 72-80.

13. A.I. Bandura, N.V. Petrechko, O.B. Skaskiv, Maximum modulus of analytic in a bidisc functions of bounded $\mathbf{L}$-index and analogue of Theorem of Hayman, Matematica Bohemica.

14. A.I. Bandura, N.V. Petrechko, Properties of power series of analytic in a bidisc functions of bounded L-index in joint variables, Carpathian Math. Publ., 9 (2017), №1, 6-12.

15. A.I. Bandura, Properties of positive continuous functions in $\mathbb{C}^{n}$, Carpathian Math. Publ., 7 (2015), №2, 137-147.

16. A.I. Bandura, Some improvements of criteria of L-index boundedness in direction, Mat. Stud., 47 (2017), №1, 27-32.

17. A. Bandura, O. Skaskiv, Entire functions of bounded L-Index: its zeros and behavior of partial logarithmic derivatives, J. Complex Analysis, Article ID 3253095, 10 p, 2017.

18. A.I. Bandura, O.B. Skaskiv, Iyer's metric space, existence theorem and entire functions of bounded L-index in joint variables, Bukovyn. Mat. Zh., 5 (2017), №3-4, 8-14. (in Ukrainian)

19. M.T. Bordulyak, A proof of Sheremeta conjecture concerning entire function of bounded l-index, Mat. Stud., 11 (1999), №2, 108-110.

20. M.T. Bordulyak, On the growth of entire solutions of linear differential equations, Mat. Stud., 13 (2000), №2, 219-223.

21. B.C. Chakraborty, R. Chanda, A class of entire functions of bounded index in several variables, J. Pure Math., 12 (1995), 16-21.

22. B.C. Chakraborty, T.K. Samanta, On entire functions of bounded index in several variables, J. Pure Math., 17 (2000), 53-71.

23. W.K. Hayman, Differential inequalities and local valency, Pacific J. Math., 44 (1973), №1, $117-137$.

24. G.J. Krishna, S.M. Shah, Functions of bounded indices in one and several complex variables, In: Mathematical essays dedicated to A.J. Macintyre, Ohio Univ. Press, Athens, Ohio, 1970, 223-235.

25. V.O. Kushnir, M.M. Sheremeta, Analytic functions of bounded l-index, Mat. Stud., 12 (1999), №1, 59-66.

26. B. Lepson, Differential equations of infinite order, hyperdirichlet series and entire functions of bounded index, Proc. Sympos. Pure Math., 2 (1968), 298-307.

27. F. Nuray, R.F. Patterson, Entire bivariate functions of exponential type, Bull. Math. Sci., 5 (2015), №2, $171-177$.

28. F. Nuray, R.F. Patterson, Multivalence of bivariate functions of bounded index, Le Matematiche, 70 (2015), №2, 225-233.

29. R. Patterson, F. Nuray, A characterization of holomorphic bivariate functions of bounded index, Mathematica Slovaca, 67 (2017), №3, 731-736.

30. L.I. Ronkin, Introduction to theory of entire functions of several variables, Nauka, Moscow, 1971, (in Russian); Engl. transl.: L.I. Ronkin, Introduction to theory of entire functions of several variables. AMS, Translations of mathematical monographs, V. 44, 1974.

31. W. Rudin, Function Theory in the unit ball on $\mathbb{C}^{n}$. - Reprint of the 1980 Edition, Springer, 2008.

32. M. Salmassi, Functions of bounded indices in several variables, Indian J. Math., 31 (1989), №3, $249-257$.

33. M.N. Sheremeta, Entire functions and Dirichlet series of bounded l-index, Russian Math. (Iz. VUZ), 36 (1992), №9, 76-82.

34. M.N. Sheremeta, A.D. Kuzyk, Logarithmic derivative and zeros of an entire function of bounded l-index, Sib. Math. J., 33 (1992), №2, 304-312.

35. M. Sheremeta, Analytic functions of bounded index, VNTL Publishers, Lviv, 1999.

36. S.N. Strochyk, M.M. Sheremeta, Analytic in the unit disc functions of bounded index, Dopov. Akad. Nauk Ukr., (1993), №1, 19-22. (in Ukrainian)

37. K. Zhu, Spaces of holomorphic functions in the unit ball, Graduate Texts in Mathematics, Springer, New York, 2005.

Ivano-Frankivsk National Technical University of Oil and Gas andriykopanytsia@gmail.com

Ivan Franko National University of Lviv

olskask@gmail.com 